Pierre G. Carlier 1, 2, 3 , Benjamin Marty, 2 , Olivier Scheidegger ${ }^{1,4}$, Paulo Loureiro de Sousa ${ }^{5}$,

Pierre-Yves Baudin ${ }^{6}$,

Eduard Snezhko ${ }^{3}$,

Dmitry Vlodavets ${ }^{2}$

${ }^{1}$ Institute of Myology,

Pitié-Salpêtrière

University Hospital, Paris,

France

${ }^{2} \mathrm{CEA}, \mathrm{DSV}, \mathrm{I}^{2} \mathrm{BM}$,

MIRCen, NMR

Laboratory, Paris, France

${ }^{3}$ National Academy

of Sciences, United

Institute for Informatics

Problems, Minsk, Belarus

${ }^{4}$ Bern University,

Inselspital, Department

of Clinical Research, Bern

Switzerland

${ }^{5}$ Strasbourg University,

CNRS, ICube, Strasbourg,

France

${ }^{6}$ Consultants

for Research in Imaging

and Spectroscopy,

Tournai, Belgium

${ }^{7}$ N.I. Prirogov Russian

National Medical Research

University, Clinical

Research Institute

of Pediatrics, Moscow,

Russian Federation

P.G. Carlier

(corresponding

author)

Institut de Myologie

and CEA, DSV, I'BM,

MIRCen, Laboratoire

de RMN,

Pitié-Salpêtrière

University Hospital,

Bd de l'Hôpital, 75651

Paris Cedex 13, France

Contact

p.carlier@institut-

myologie.org

\section{Imagerie et spectroscopie par résonance magnétique nucléaire du muscle strié squelettique}

\section{Utilisation comme outil d'évaluation pour les essais cliniques}

\author{
Pierre G. Carlier, Benjamin Marty, Olivier Scheidegger, Paulo Loureiro de Sousa, \\ Pierre-Yves Baudin, Eduard Snezhko, Dmitry Vlodavets
}

Au cours des dernières années, les traitements de nombreuses maladies neuromusculaires, jusqu'ici incurables, ont bénéficié d'importants progrès. Ce bouleversement contextuel a eu pour conséquence de stimuler le développement de nouveaux outils d'évaluation atraumatiques. Ceux-ci peuvent être classés en trois grandes catégories : les explorations fonctionnelles musculaires, les marqueurs des fluides biologiques et l'imagerie musculaire. Au sein de cette dernière, l'imagerie par résonance magnétique nucléaire (IRMN) offre un très large éventail de possibilités pour caractériser la composition, la fonction et le métabolisme du muscle strié squelettique. Aujourd'hui, trois indicateurs RMN sont couramment intégrés dans les protocoles de recherche clinique : 1) le volume musculaire ou l'aire d'une section musculaire transversale ciblée, 2) le pourcentage de graisse intramusculaire et 3) le T2 de l'eau musculaire. Ils permettent de quantifier respectivement la trophicité du muscle, les dégénérescences graisseuses chroniques et l'œedème tissulaire (ou plus généralement "l'activité de la maladie "). Un quatrième indicateur, le volume de tissu contractile est facilement dérivable des deux premiers. Les cartographies de fraction graisseuse, souvent issues de séquences Dixon, ont fait la preuve de leur utilité pour détecter de subtils changements de composition musculaire et se sont, à plusieurs reprises, révélées plus sensibles que les évaluations fonctionnelles standards. Cet indicateur sera probablement le premier parmi ceux proposés à être validé comme paramètre principal par les organismes de régulation. La diversité des contrastes obtenus par RMN permet d'explorer de nombreuses autres pistes de caractérisation du muscle squelettique et de nouveaux biomarqueurs RMN sont à attendre dans un avenir plus ou moins proche. Des séquences à TE ultra-courts (UTE), le rehaussement tardif post-injection de gadolinium et l'élastographie par RMN sont en cours d'étude pour l'évaluation de la fibrose interstitielle du muscle squelettique. De nombreuses options existent pour mesurer la perfusion et l'oxygénation du muscle par RMN. La RMN de diffusion ainsi que l'utilisation d'algorithmes d'analyse de texture pourraient apporter des informations supplémentaires sur l'organisation musculaire aux échelles respectivement microscopiques et mésoscopiques. La spectroscopie RMN du phosphore ${ }^{31} \mathrm{P}$ est la technique de référence pour l'évaluation atraumatique de l'énergétique musculaire pendant et après exercice. Le spectre ${ }^{31} \mathrm{P}$ du muscle dystrophique au repos est notablement altéré, et plusieurs de ses résonances informent sur l'intégrité de la membrane cellulaire. D'importants efforts sont consacrés à l'accélération de l'acquisition des images au travers plusieurs approches, allant de l'extraction du contenu en graisse et des cartographies T2 au départ d'une unique séquence, jusqu'à l'utilisation de scénarios d'acquisition partielle des matrices. Dans un avenir proche, une diminution spectaculaire du temps d'acquisition est attendue. Cela renforcera l'attractivité des indicateurs RMN et facilitera leur intégration aux essais de recherche clinique. 


\section{Motivations à l'utilisation de la RMN comme outil de mesure pour les maladies neuro-musculaires}

La vision du corps médical sur les maladies neuromusculaires s'est radicalement transformée au cours des deux dernières décennies. La majorité de ces pathologies est d'origine génétique et elles étaient jusqu'à présent peu curables. Les traitements disponibles étaient essentiellement de soutien ou palliatifs. Appartenant toutes à la catégorie des maladies rares, elles sont longtemps restées hors du champ de la recherche menée par l'industrie pharmaceutique. Aujourd'hui, les maladies rares ont été repositionnées au cour des préoccupations, avec d'importants programmes de recherche initiés et financés par des institutions publiques au niveau transnational. Plus déterminant encore, les progrès de la thérapie génique et de la pharmacogénétique sont sur le point, si ce n'est déjà fait, de révolutionner l'impact de ces maladies sur la vie des patients (Muntoni et Wood 2011; Cirak et al. 2011 ; Arechavala-Gomeza et al. 2012 ; Erriquez et al. 2013 ; Scotter et Shaw 2013; Douglas et Wood 2013 ; Mercuri et Muntoni 2013 ; Touznik et al. 2014 ; Bushby et al. 2014 ; Voit et al. 2014 ; Buyse et al. 2015 ; Blat et Blat 2015).

Suite à ces innovations thérapeutiques, de nouveaux besoins ont rapidement émergé, dont la nécessité de contrôler l'effet des traitements sur les muscles au cours du temps. Pour ce faire, de nouveaux outils de mesure étaient devenus nécessaires, et qui seraient, idéalement, atraumatiques, peu coûteux et fournissant des résultats faciles à interpréter. On peut en distinguer trois catégories: les outils fonctionnels, les biomarqueurs de fluides biologiques et l'imagerie. Les études fonctionnelles sont au premier plan, avec une variété de dispositifs et de protocoles, dont beaucoup sont optimisés pour la mesure de mouvements spécifiques, et d'autres visant plutôt à une évaluation de l'activité globale du patient (Scott et al. 2012; Mazzone et al. 2012; Servais et al. 2013 ; McDonald et al. 2013 ; Mayhew et al. 2013 ; Pane et al. 2014 ; Mazzone et al. 2014 ; Hogrel et al. 2014 ; Decostre et al. 2015 ; Lynn et al. 2015; Seferian et al. 2015a ; Seferian et al. 2015b). Ces derniers outils font partie d'une nouvelle discipline en pleine expansion, l'actimétrie, et offrent un accès inédit à une évaluation du patient dans son environnement personnel sur de longues périodes de temps. Autre innovation parmi ces outils, les marqueurs des fluides biologiques ont commencé à faire preuve de leur utilité, en particulier depuis la découverte et l'exploitation des $\mu$ RNA. Enfin, troisième grande classe d'outils de mesure, l'imagerie est de plus en plus utilisée, bien qu'elle requière d'importants investissements en équipements (les appareils à ultra-sons les plus performants ne faisant pas exception). En outre, les méthodes tomographiques, la tomodensitométrie (CT) et la résonance magnétique nucléaire $(\mathrm{RMN})$, sont pénalisées par leur absence de portabilité. Néanmoins, la RMN est la seule technique qui permet d'évaluer l'anatomie, la composition et la fonction du muscle au cours d'un même examen. Ces facultés de la RMN sont aujourd'hui de mieux en mieux comprises et appréciées, comme en témoignent les recommandations en matière d'imagerie émises par les instances régulatrices, EMA et FDA, pour l'homologation de nouveaux médicaments (voir Figure 1).

Dans cette revue, les auteurs essaient de donner une vue d'ensemble complète des études où le rôle d'outil d'évaluation de la RMN est évalué dans les faits ou discuté comme perspective d'avenir. Plutôt que de proposer un catalogue objectif mais impersonnel, ils ont choisi délibérément de faire part de leur commentaires et opinions en vue de guider le lecteur intéressé par une mise en pratique de l'imagerie quantitative. La part subjective de ce travail est pleinement assumée.

\section{La quantification comme prérequis à l'utilisation de la RMN comme biomarqueur}

Un indicateur de substitution (surrogate marker), d'une maladie ou d'une condition quelle qu'elle soit doit pouvoir, afin d'être utilisé comme biomarqueur (Vasan 2006 ; Strimbu et Tavel 2010) être exprimé comme une mesure quantifiée et faire la preuve de sa capacité à refléter des événements pathologiques spécifiques avec exactitude et précision. Pour y satisfaire, l'imagerie médicale a dû suivre un long processus d'évolution pour adjoindre une dimension quantitative aux habituelles descriptions qualitatives des maladies. Cela a été possible grâce à un apport constant d'innovations technologiques qui a donné lieu à de spectaculaires améliorations des appareils en termes de stabilité et versatilité, ainsi qu'au méticuleux développement de protocoles dédiés (Tofts 2003). Cette évolution a profité à tous les systèmes et les organes, y compris le muscle. Pour les pathologies musculaires, comme pour les autres maladies, les bénéfices sont multiples: une meilleure estimation de la sévérité de l'atteinte, la possibilité de surveiller la progression des altérations au cours du temps et, plus important encore, d'évaluer la réponse du muscle à une intervention thérapeutique (voir Figure 2). 


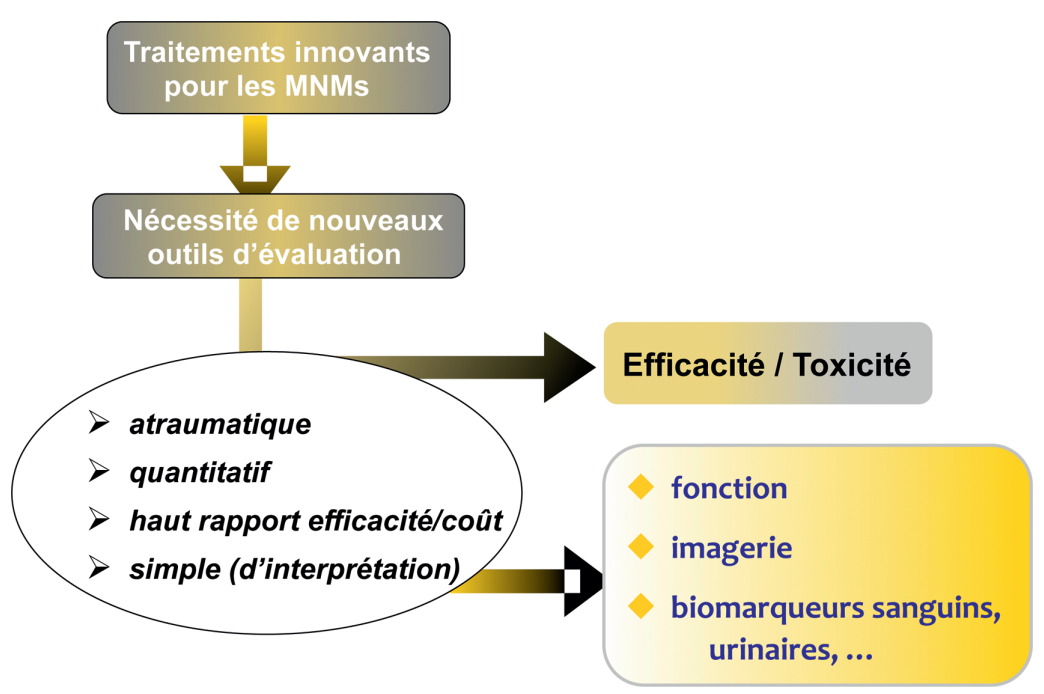

Figure 1

La découverte et l'introduction de traitements causaux pour un nombre croissant de maladies neuro-musculaires a généré des besoins nouveaux en matière d'outils d'évaluation pour

s'assurer tant de l'efficacité que de l'innocuité de ces nouvelles options thérapeutiques. Au rang de ceux-ci, l'imagerie musculaire, en particulier par RMN, occupe une place de choix.

\section{Qualitative Quantitative}

\section{- Trophicité}

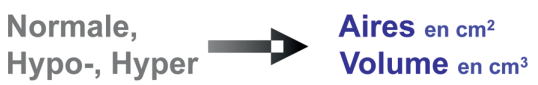

(T1w) (Opposition de phase
de Dixon)

\section{- Lésions dégénératives chroniques}

\# remplacement graisseux

\# fibrose
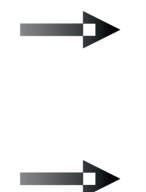

$\%$ Infiltration graisseuse (Eau-Graisse « Dixon »)

$\%$ Collagène interstitiel (??UTE, ?? DEMRI)

\section{- « Activité de} Fat Sat-T2w la maladie » (STIR)

Figure 2

Comparaison des approches qualitatives et quantitatives de l'imagerie musculaire par résonance magnétique nucléaire.

Par un parcours fort similaire à ce qui s'est passé pour la plupart des organes, la RMN est devenue un acteur pivot dans l'imagerie quantitative des muscles squelettiques (Hollingsworth et al. 2012). Les variables et indices quantitatifs obtenus par imagerie et spectroscopie RMN sont les meilleurs candidats de l'imagerie médicale en vue d'une utilisation comme bio-marqueur ou outils d'évaluation dans les essais cliniques dédiés aux pathologies musculaires.
Les outils de mesure par RMN actuellement disponibles

L'imagerie et la spectroscopie par RMN peuvent générer quantité d'informations pertinentes sur l'anatomie du muscle, sa structure ou sa composition, sa physiologie et sa biochimie. Bien qu'un nombre important de variables RMN soient en cours de test et que leur place comme biomarqueur soit à l'étude, seules trois d'entre elles sont communément acceptées, sinon totalement validées, pour le suivi longitudinal du muscle squelettique. Elles sont quasi-systématiquement intégrées à la préparation des nouveaux essais thérapeutiques. Elles visent à évaluer :

- la trophicité du muscle, via des mesures de surface et de volume,

- le degré de dégénérescence musculaire via la fraction de graisse du signal RMN dans le muscle,

- l'activité de la maladie dans les pathologies évolutives, via le temps de relaxation T2 de l'eau musculaire (voir Figure 2).

\section{La trophicité du muscle squelettique}

Combinant une haute résolution spatiale, une dynamique de signal élevée, la possibilité d'acquérir en $3 \mathrm{D}$, et des algorithmes efficaces de correction de distorsions (surtout dues à une non-linéarité des gradients), l'imagerie par RMN est de facto devenue la méthode anatomique de référence pour mesurer volumes et dimensions d'organes (Hunter et al. 2011 ; Jovicich et al. 2013; Mills et Tamnes 2014 ; Xi et al. 2014). Ce constat vaut au moins autant pour les muscles striés squelettiques que pour les autres organes. Même si l'exactitude des mesures est rarement évaluée, car nécessitant des autopsies, d'excellents résultats furent rapportés dans les cas où elle put être déterminée (Mitsiopoulos et al. 1998). En outre, il a été systématiquement montré que la reproductivité et le pouvoir discriminant de l'imagerie par RMN sont très élevés (Wagner et al. 2008 ; Fischmann et al. 2013; Barnouin et al. 2014 ; Thomas et al. 2014), au moins autant qu'avec les ultrasons (Lima et al. 2012) ou la tomodensitométrie aux rayons X (Strandberg et al. 2010), et avec l'avantage d'une caractérisation tissulaire (voir paragraphe ci-dessous) très supérieure. Les exemples suivants illustrent la capacité de la RMN à identifier de subtils changements de trophicité du muscle. Suite à une injection de toxine botulique dans le gastrocnémien d'enfants infirmes moteurs cérébraux, une diminution de la masse de ce muscle de $4 \%$ fut mesurée, compensée par une augmentation de $4 \%$ de la masse du soléaire (Williams et al. 2013). Le coefficient de variation de l'estimation du volume des muscles des avant-bras oscille entre 0,8 et $5,7 \%$, suivant le muscle 
considéré (Smeulders et al. 2010). Les effets d'un "désentraînement " faisant suite à des semaines de protocoles d'exercices concentriques et excentriques combinés à des restrictions de perfusion ont été évalués et ont montré une dépendance de la perte de trophicité au protocole d'entraînement (Yasuda et al. 2015).

Dans la plupart des cas, la variable retenue est la trophicité du muscle plutôt que son volume. Une fois mesuré, le volume d'un muscle est normalisé par sa longueur, ou plus souvent par la longueur de l'os adjacent, afin d'obtenir un indice de trophicité.

Pour un muscle pathologique, il est préférable de baser l'indice de trophicité sur la masse vraie de tissu contractile plutôt que sur le volume global du muscle. L'indice de masse contractile est calculé avec la formule suivante : volume du muscle (ou aire de la section transversale) $\mathrm{x}(1$ - fraction graisseuse), cette dernière provenant de l'analyse de l'imagerie eaugraisse (voir paragraphe suivant) (Wokke et al. 2014b).

Chez les garçons atteints de la maladie de Duchenne, le volume contractile des différents muscles mesurés séparément, a montré une relation complexe avec la diminution de la force du muscle correspondant. Cette dernière était proportionnelle à la perte musculaire au niveau du quadriceps, mais beaucoup plus importante qu'attendu par la seule

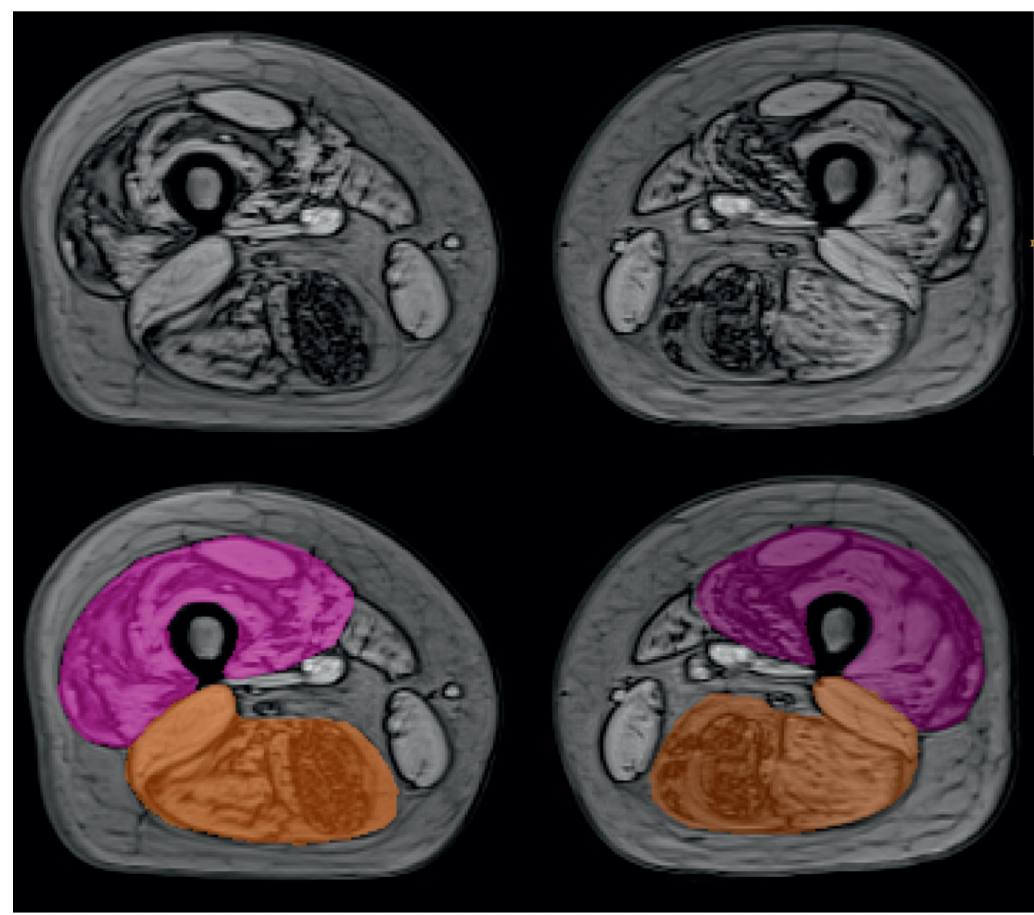

Figure 3

Exemple de segmentation manuelle du quadriceps et des ischio-jambiers, réalisée sur des images en opposition de phase d'une acquisition de type Dixon. perte de tissu contractile dans les ischio-jambiers et le tibial antérieur (Wokke et al. 2014b).

Dans le muscle normal, ou quand la pathologie affecte le muscle de façon homogène, les aires de sections transversales mesurées à des hauteurs bien définies, par ex. à la moitié de la cuisse, fourniront des indices de trophicité pertinents (Morse et al. 2007 ; Tanaka et Kanehisa 2014 ; Hogrel et al. 2015) tout en réduisant considérablement les durées d'acquisition et surtout de post-traitement.

Toutefois, cette approche implique un positionnement rigoureux fondé sur des repères anatomiques. Ces repères peuvent être identifiés extérieurement, tels l'épine iliaque antéro-supérieure ou le bord supérieur de la rotule, ou partant d'images de repérage acquises au préalable (Fischmann et al. 2014). Les repères externes ont en général la préférence, pour pallier l'éventuelle difficulté à connaître la position de la table de la machine lorsque l'on passe d'une configuration d'antennes à une autre. Quoiqu'il en soit, il est bien plus aisé de compenser les variations longitudinales de positionnement entre examens successifs lorsque les structures osseuses sont entièrement présentes dans l'image. Il a été montré que la reproductibilité inter-examen de la mesure de l'aire de la section transverse est meilleure si l'on utilise des images de repérage plutôt que des repères anatomiques externes (Fischmann et al. 2014). Lorsque le processus de dégénérescence musculaire dû à la pathologie est particulier, par ex. progressant de distal en proximal, ou lorsque qu'il est inconnu, il est hautement préférable d'acquérir des volumes, ou d'assez grandes séries de coupes, couvrant la majeure partie du muscle.

Des problèmes supplémentaires surviennent avec la population pédiatrique, liés au processus de croissance. Lorsque l'intervalle de temps entre les examens est suffisant pour que la croissance montre ses effets, la mise en relation des coupes d'imagerie d'un examen à l'autre n'est pas simple à décider. Faute d'une meilleure solution, l'espacement entre les coupes est ajusté proportionnellement à l'allongement osseux. Avec des acquisitions 3D, cet ajustement peut être réalisé en post-traitement. S'il s'agit de séries de coupes 2D, l'écartement entre les coupes doit être augmenté en proportion de la croissance mesurée; une action rarement entreprise étant donné qu'elle requiert une intervention au moment de l'acquisition elle-même.

En pratique, excepté pour certaines études spécifiques, une évaluation approfondie de la trophicité musculaire n'a, à ce jour, été que très rarement entreprise. Cela exige la patience de délimiter chaque muscle individuellement sur l'image, ce qui aujourd'hui doit être effectué à la main (voir 
Figure 3). C'est une tâche longue, fastidieuse et peu gratifiante, ce qui explique pourquoi l'étude de la trophicité musculaire est le plus souvent laissée de côté. Très peu d'études longitudinales de la trophicité du muscle ont été réalisées sur des patients atteints de maladies neuro-musculaires. Un effet positif de l'enzymothérapie de substitution sur le volume musculaire des membres inférieurs a été observé chez des patients Pompe après seulement 6 mois de traitement (Ravaglia et al. 2010). Suite à une période d'observation de 6 mois, l'aire de la section transversale du mollet de patients atteints de myosite à inclusions a perdu $6,5 \%$, alors qu'aucun changement n'a été observé chez des patients Charcot-Marie-Tooth 1A (Morrow et al. 2015). Sur une cohorte réduite, et avec une méthodologie discutable, aucun changement de trophicité musculaire n'a été détecté dans les membres inférieurs de patients atteints d'une amyotrophie spinale (Sproule et al. 2011)

Des essais de développement d'outils de segmentation automatique ont été entrepris, mais très peu d'entre eux sont parvenus à générer des résultats fiables, et aucun n'a encore permis d'analyser des images de muscles pathologiques présentant des infiltrations graisseuses. Très récemment, il a été rapporté qu'un logiciel de segmentation automatique s'appuyant sur un modèle générait des résultats très proches d'une segmentation manuelle (Karlsson et al. 2015). Une approche naïve a également été proposée, où une estimation de la masse musculaire globale est calculée à partir de l'histogramme d'intensité de segments entiers de membres (Mattei et al. 2006). Cela n'offrirait que peu d'avantages par comparaison à de simples mesures

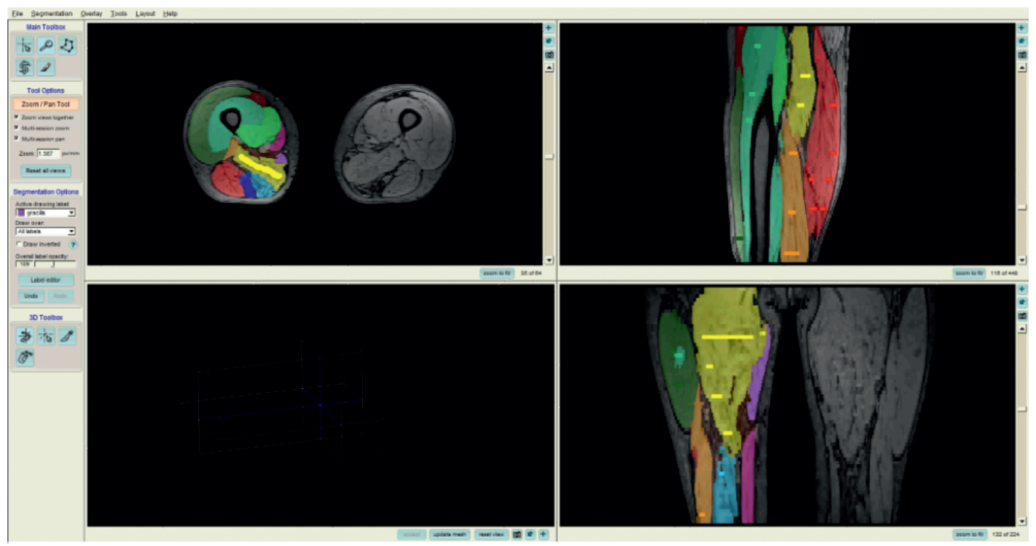

Figure 4

Accélération considérable de la segmentation musculaire par l'utilisation d'un logiciel interactif qui détecte les contours des muscles, assisté par quelques repères tracés par l'utilisateur. Les quelques traits tracés par l'utilisateur apparaissent en surbrillance sur les images. Le temps nécessaire pour la segmentation des muscles de la cuisse passe de plusieurs dizaines de minutes à quelques minutes. externes, et ignorerait le fait que les muscles ne sont habituellement pas atteints de façon homogène par la maladie. Dans la cuisse de chien GRMD, suivant le muscle considéré, il a été montré que la trophicité déduite du volume de muscle rapporté à la masse totale pouvait augmenter, rester stable ou diminuer avec l'âge (Kornegay et al. 2012). Si l'on se concentre sur les muscles modérément ou sévèrement atteints mais non entièrement détruits, la capacité à détecter une inflexion dans la progression de maladie s'en trouvera renforcée ; à l'inverse, par un effet de dilution avec les muscles peu atteints, une mesure globale voit sa sensibilité aux changements décroître. Une approche plus réaliste consisterait à se tourner vers des logiciels de segmentation interactive. Ici, l'implication d'un opérateur reste obligatoire mais, en pratique, c'est également le cas avec une méthode tout automatique, où il est nécessaire de parcourir le volume segmenté pour repérer les erreurs. Au moins un logiciel fonctionnant selon ce principe est en cours de développement, s'appuyant sur l'algorithme dit des marches aléatoires (Carlier et al. 2014). Ici, il suffit à l'utilisateur de marquer grossièrement chaque muscle, toute les cinq ou dix coupes. Si les contours sont incorrectement détectés par l'algorithme, l'utilisateur n'a qu'à ajouter un coup de pinceau supplémentaire dans la région mal étiquetée. Des tests effectués sur les muscles de la cuisse ont montré que cette approche est comparable à une segmentation manuelle, que ce soit en termes de volume musculaire ou de variabilité inter-opérateur, tout en réduisant d'au moins un facteur dix le temps de travail (voir Figure 4). Une plus grande efficacité pourrait encore être atteinte en améliorant la réactivité du logiciel aux interactions, voire en introduisant une connaissance à priori de la forme des muscle ou de leur position relative.

La source de la difficulté à segmenter des images de muscles, ainsi que la principale cause de l'échec des méthodes automatiques, est l'absence fréquente de contours visibles séparant les muscles. Ceci est particulièrement vrai avec des images pondérées $\mathrm{T} 1$ standards ou pondérées T2 en écho de spin. Effectuer la segmentation sur des images possédant un bon contraste entre le tissu musculaire et les fascias est une manière d'améliorer la situation; c'est par exemple le cas avec des images en écho de gradient acquises à certains temps d'écho spécifiques de telle sorte que les signaux de l'eau et de la graisse se retrouvent en opposition de phase (voir ci-dessous). Dans notre laboratoire, les mesures de trophicité sont systématiquement réalisées sur ce type d'images (voir Figure 3). Les tentatives d'utilisation 
de séquences d'imagerie de susceptibilité dédiées pour renforcer davantage le contraste entre les fascias et les aponévroses ont pour le moment échoué. L'absence fréquente de lésions dégénératives dans la population pédiatrique aux premiers temps de la maladie constitue une motivation supplémentaire pour disposer de mesures fines de la trophicité musculaire. Des neuro-pédiatres ont émis l'hypothèse que des altérations relativement légères de la trophicité pourraient être un indicateur précoce de l'atteinte musculaire (Robert Carlier et Susana Quijano-Roy, communication personnelle). La preuve doit en être apportée et l'accès à des méthodes de segmentation rapides et simples permettra d'en vérifier le bien-fondé.

\section{Les altérations dégénératives chroniques}

Les dommages chroniques aux myocytes mais aussi les anomalies structurelles des fibres ont pour résultat à long terme le remplacement du tissu contractile par de la graisse et/ou du tissu conjonctif. L'imagerie de la fibrose reste une question épineuse, qui sera traitée dans un autre paragraphe. À l'opposé, les changements dégénératifs graisseux sont facilement détectés et quantifiés, en tirant parti des différences entre les fréquences de résonance (déplacement chimique) ou encore entre les taux de relaxation T2, voire même T1 des noyaux d'hydrogène de l'eau et des lipides. Pour une revue technique exhaustive, voir Bley et al. ou Hu et Kan (Bley et al. 2010 ; Hu et Kan 2013).

Une classification visuelle de l'infiltration graisseuse sur des images pondérées en T1, par exemple à l'aide de l'échelle de Lamminen-Mercuri (Lamminen 1990), est suffisante à des fins diagnostiques mais est totalement inappropriée pour monitorer la progression des lésions dégénératives, qui est considérablement plus subtile à apprécier. À supposer que l'œil humain puisse classer les lésions dégénératives graisseuses entre 1 et 4 sans jamais se tromper, ce qui n'est certainement pas le cas, un changement de grade surviendrait en moyenne pour une augmentation de $17.6 \%$ de la fraction graisseuse. Même les formes les plus sévères de dystrophie ne s'acompagnent pas d'un tel taux annuel de destruction musculaire. L'étude récemment réalisée par Willis et al. (Willis et al. 2013) a démontré sans ambiguïté l'impossibilité d'évaluer au moyen de la classification de Lamminen-Mercuri la progression de la dystrophie musculaire des ceintures chez des patients adultes atteints de la forme I. Il est parfois proposé comme solution d'afficher sur le même écran des images $\mathrm{T} 1 \mathrm{w}$ acquises à différents moments durant le suivi du patient et de les confronter une à une, muscle par muscle. Procéder de la sorte permet probablement de détecter des changements mais demeure hautement dépendant de l'observateur, avec un seuil de sensibilité inconnu et sans quantification, indispensable pour pouvoir comparer ou patients ou interventions. Des tentatives ont été faites il y a un certain temps (LeroyWillig etal. 1997) mais aussi plus récemment (Pichiecchio et al. 2002 ; Mattei et al. 2006) pour séparer l'eau et la graisse par un seuillage appliqué à des images de routine pondérées en $\mathrm{T} 1$, en essayant de tirer avantage de l'apparente simplicité de cette approche. Certains travaux ont tenté de prendre en compte la possible co-existence de graisse et d'eau dans le même voxel et ont calculé la fraction graisseuse en utilisant une combinaison linéaire de signaux de graisse pure et de muscle pur (Leroy-Willig et al. 1997). Les études plus récentes ont juste opéré un classement binaire des voxels de la graisse et du muscle, ce qui est totalement inadapté à l'évaluation de l'infiltration graisseuse dans un contexte de maladie musculaire chronique (Pichiecchio et al. 2002; Mattei et al. 2006). Toutes ces approches basées sur l'utilisation d'images standards T1w supposent un émetteur radio-fréquence ainsi qu'une réception parfaitement ou quasi-parfaitement homogènes, ce qui n'est jamais le cas. C'était un postulat à peu près acceptable par le passé lorsqu'il était habituel de travailler sur des imageurs à faible à champ magnétique faible, $0.5 \mathrm{~T}$. Ce n'est plus le cas, pour les imageurs à haut champ, 3T ou plus, ou quand la réception du signal s'effectue via plusieurs rangées de bobines de surfaces. Il faut espérer qu'un jour des solutions techniques seront mises en œuvre et garantiront une très grande homogénéité d'émission et de réception et/ou des corrections parfaites de leurs imperfections en post-traitement, En attendant, nous déconseillons fortement le recours à une technique de séparation de la graisse et de l'eau musculaire basée sur des profils d'intensité de l'image. Cette approche est particulièrement trompeuse pour les non-experts qui pourraient être abusés par l'apparente simplicité de la méthode.

Pour le suivi des changements dégénératifs chroniques musculaires, on préfère aujourd'hui les séquences d'imagerie eau-graisse, le plus souvent appelées séquences Dixon (Ma 2008) (voir Figure 5). Elles mettent à profit le glissement de phase qui se développe progressivement entre le signal de l'eau et celui de la graisse au cours du temps d'évolution d'une séquence en écho de gradient. Le principal avantage de cette approche est que la séparation de l'eau et des graisses est, au premier ordre, indépendante de l'homogénéité du champ magnétique. Une conséquence importante 


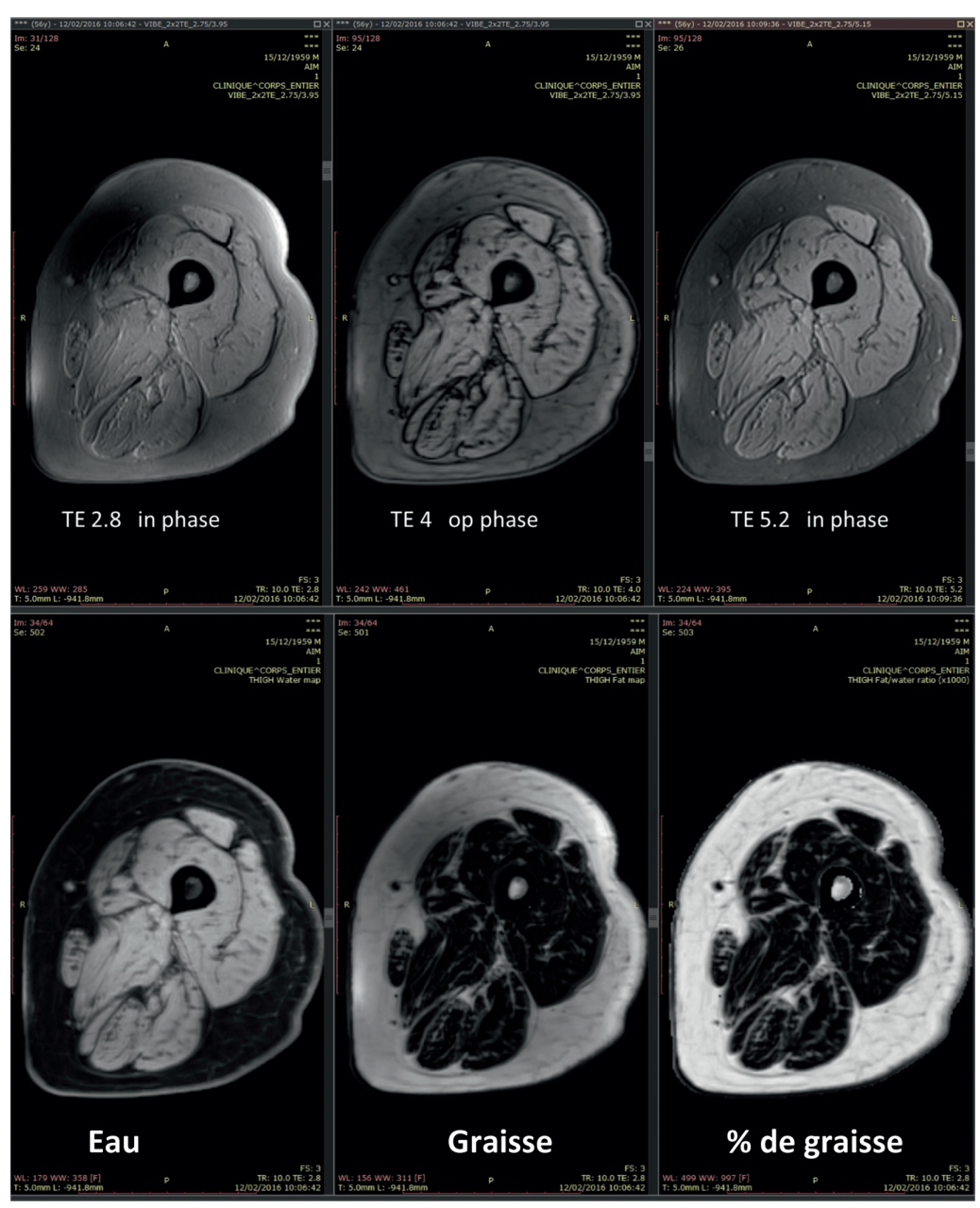

Figure 5

Exemple d'imagerie eau-graisse musculaire. Sur le panneau supérieur, les 3 images acquises par la séquence Dixon standard, avec l'eau et la graisse successivement enphase, en opposition de phase et à nouveau en phase. Sur le panneau inférieur, les 3 images calculées à partir des images natives, l'image de l'eau, celle de la graisse, et à droite, celle du pourcentage de graisse apparent.

est, qu'avec cette technique, il est possible d'explorer sans difficulté de grands champs de vue qui couvrent de grands volumes.

Dans la version standard du Dixon, seulement la résonance des méthylènes des lipides est prise en compte. Deux, ou mieux, trois images avec la graisse et l'eau successivement en phase et en opposition sont acquises et permettent l'extraction des composantes aqueuses et lipidiques. L'imagerie Dixon à deux points (éventuellement "élargie") génère des résultats satisfaisants au niveau du foie. Cependant, pour des applications musculaires, des attributions erronées du signal de l'eau et la graisse peuvent se produire entre membres ou à l'intérieur de segments de membres, cette dernière étant particulièrement problématique (Hollingsworth et al. 2012). L'utilisation de séquences d'imagerie Dixon dite à trois points met presque toujours à l'abri de cet écueil.
Ignorer les autres résonances lipidiques génère un certain degré d'imprécision (Wokke et al. 2013). Celle-ci peut être réduite par une modélisation plus complète du spectre des lipides, habituellement avec 3 ou 4 résonances principales, ce qui nécessite la collecte de 6 échos et des calculs relativement lourds (Hu et al. 2012). Cette approche dénommée "décomposition itérative de l'eau et des graisses avec un écho asymétrique et une estimation par la méthode des moindres carrées" (IDEAL) et T2*-IDEAL, constitue la méthode la plus avancée actuellement disponible pour réaliser une imagerie eau-graisse. L'acquisition d'échos multiples implique un allongement des temps de répétition, ainsi que l'application de corrections pour les effets liés au T2*. La méthode IDEAL avec des échos multiples est celle qui mesure le plus précisément le pool d'hydrogène de la fraction graisseuse. Elle possède la capacité d'identifier d'éventuelles modifications du spectre lipidique, que celles-ci soient induites par l'alimentation ou par une pathologie. Il y a peu d'indications que ce soit le cas au niveau du muscle strié squelettique, ou si ces différences existent, elles sont de faible amplitude et difficilement détectables dans les conditions habituelles.

Si les intensités relatives du spectre lipidique dans les muscles infiltrés peuvent être considérées comme étant indépendantes de l'état du patient, ce qui semble une hypothèse raisonnable, il n'est pas nécessaire de passer par une acquisition avec 6 échos. Un facteur de correction linéaire peut être appliqué au signal de la graisse et la fraction de graisse exacte sera obtenue à partir d'une mesure standard à 3 points. Le facteur de correction calculé pour les conditions de notre laboratoire est de 1.82 (Azzabou et al. 2015c).

La façon d'exprimer la teneur en graisse musculaire peut également varier. On peut simplement regarder le pourcentage du signal RMN attribuable à la graisse dans le voxel ou dans le muscle. En fonction du temps de répétition des images et du temps d'écho choisis, des corrections pour les effets des pondérations T1 et T2 peuvent être appliquées. On peut aller plus loin et tenter d'exprimer le contenu graisseux en grammes de lipides par unité de masse ou de volume musculaire. Ces procédures ont été développées pour le foie et nécessitent des hypothèses supplémentaires ou des mesures de la composition en lipides du tissu (Longo et al. 1995). Comme déjà souligné, être et rester cohérent au plan méthodologique est la clé du succès dans les essais cliniques. La simplicité est un facteur vital pour assurer cette cohérence. Gardant cela à l'esprit, au moment d'écrire ces lignes, nous recommandons toujours pour l'imagerie eau-graisse du muscle 
malade la méthode Dixon à 3 points, en 3D quand cela est possible, avec une forte pondération en densité de protons (par exemple, TR de $10 \mathrm{~ms}$ et un angle de bascule de $3^{\circ}$ ) (Hollingsworth et al. 2012). Le facteur de correction pour prendre en compte le spectre des lipides peut ou ne pas être appliqué et, en première intention, des cartes paramétriques du seul pourcentage de graisses sont générées. En procédant de la sorte, l'exactitude de la mesure peut être légèrement altérée mais pas la précision ou le pouvoir discriminant, qui sont les plus importants dans le cadre d'une étude longitudinale, qu'elle comporte ou non une intervention thérapeutique.

Lorsque la teneur en graisse intramusculaire est faible, il n'est pas nécessaire de déployer des processus hasardeux et compliqués (Kim et al. 2014 ; Azzabou et Carlier 2014). La détection des lipides peut facilement être sensibilisée en diminuant le TR dans le Dixon en 2D ou en augmentant l'angle de bascule en Dixon 3D tandis que l'exactitude de la fraction relative sera préservée par l'utilisation de facteurs de correction pour la saturation de l'eau.

Bien que la séparation eau-graisse basée sur la différence des déplacements chimiques soit la méthode acceptée suivant les règles de l'état de l'art, pour évaluer l'infiltration graisseuse tissulaire, de nombreux groupes cliniques continuent à utiliser la décroissance mono-exponentielle du T2 musculaire (Garrood et al. 2009; Kim et al. 2010 ; Forbes et al. 2014; Willcocks et al. 2014 ; Kim et al. 2015). En l'absence de lipides mobiles dans les
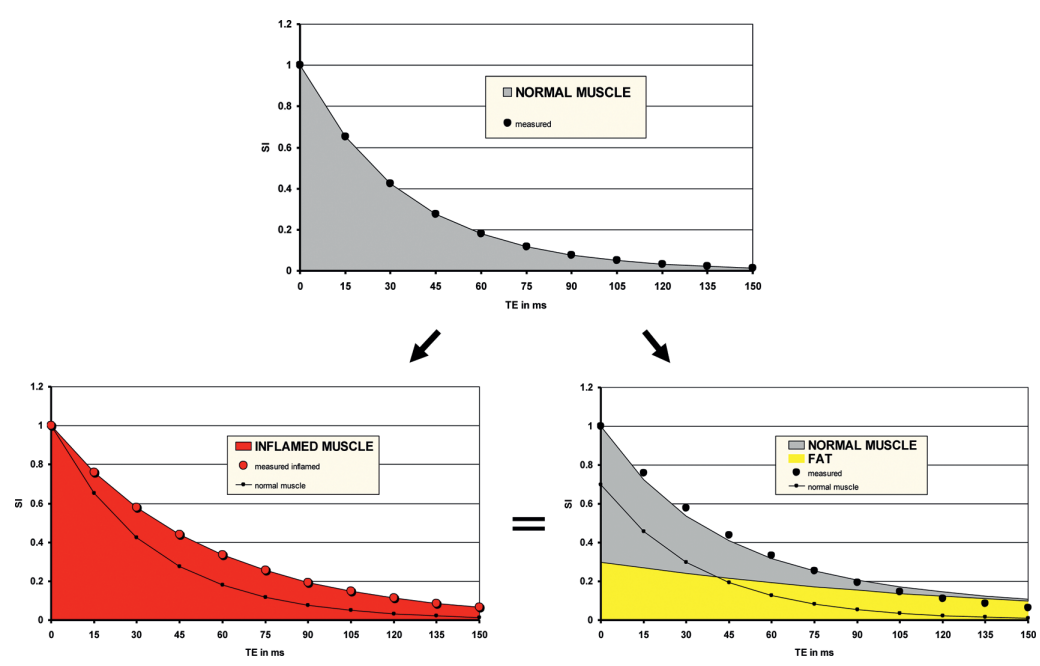

Figure 6

Le T2 global d'un muscle peut être augmenté aussi bien par la présence de lésions inflammatoires (en rouge à gauche) que d'un remplacement graisseux lors de processus dégénératifs chroniques (en jaune à droite). La simple mesure d'un T2 musculaire global par l'ajustement d'une exponentielle sur la décroissance T2 du signal ne permet pas de distinguer les deux processus. tissus, un T2 élevé pointe vers une inflammation ou un œedème tissulaire. L'augmentation du T2 due à l'inflammation ou aux œedèmes dépasse rarement 5 à $10 \mathrm{~ms}$. Mais lorsque des changements dégénératifs graisseux sont présents, le T2 des lipides étant très sensiblement plus long que celui de l'eau musculaire, l'ajustement mono-exponentiel de la décroissance du T2 musculaire est largement déterminé par le degré d'infiltration graisseuse et le T2 global du muscle devient essentiellement une mesure du contenu en lipides du muscle (Carlier 2014) (voir Figure 6). On démontre l'étroite corrélation entre le T2 global et la fraction de graisse calculée à partir des images Dixon (Azzabou et al. 2015c) ou la fraction lipidique mesurée par une spectroscopie $1 \mathrm{H}$ localisée (Kim et al. 2015).

Qu'elle soit basée sur une véritable séparation eaugraisse ou qu'elle soit estimée à partir de changement global du T2, l'évaluation de l'étendue des changements dégénératifs chroniques musculaires peut être précisément réalisée, de même que l'évolution de la maladie et les réponses aux traitements peuvent être finement monitorées. Cela a été démontré dans un grand nombre de maladies neuromusculaires.

Au niveau de la cuisse des patients atteints de la maladie de Duchenne, le taux moyen de progression des dégénérescences graisseuses a été mesuré à $5 \%$ par an. Lorsque le pourcentage de graisse atteint $50 \%$, ce signe a une valeur prédictive élevée de la perte de la marche dans l'année (Fischmann et al. 2013). Sur une petite cohorte de 3 adultes atteints de la maladie de Becker, le taux annuel d'infiltration graisseuse a été évalué à $3.7 \%$ dans la cuisse (Bonati et al. 2015b). En combinant les pourcentages de graisse avec une segmentation manuelle des muscles, on a pu déterminer des indices de masse contractile chez les patients atteints de la maladie de Duchenne (Wokke et al. 2014b). La confrontation des cartographies de pourcentages de graisses au classement de Lamminen a montré une surestimation systématique des changements dégénératifs avec les méthodes qualitatives (Wokke et al. 2013).

Toujours dans la maladie de Duchenne, l'exploration des avant-bras en imagerie a montré une implication plus importante des muscles fléchisseurs que des extenseurs, et une progression plus rapide des lésions dégénératives graisseuses chez les patients ayant perdu l'ambulation que chez les patients ambulants (Wary et al. 2015b ; Hogrel et al. 2016). L'administration de corticostéroïdes pendant une année à de jeunes garçons qui sont atteints de la maladie de Duchenne a arrêté le processus d'infiltration de la graisse dans la cuisse et la jambe tandis 
que le taux d'infiltration graisseuse était respectivement de $7 \%$ et $3 \%$ chez les enfants non-traités (Arpan et al. 2014). La gravité de la maladie a facilité la détection d'une progression des lésions dégénératives au bout de 18 mois, cette fois basée uniquement sur des mesures d'intensité d'une imagerie pondérée en T1, avec également la démonstration d'une grande variabilité inter-individuelle et intermusculaire (Hollingsworth et al. 2013a). Une telle approche est néanmoins obsolète au regard des capacités de l'imagerie quantitative et ne peut pas être recommandée pour de futurs protocoles. La même méthodologie avait été utilisée précédemment, en combinaison avec des mesures globales du T2 pour décrire les implications variables de différents territoires musculaires chez 5 patients atteints de la maladie de Duchenne (Garrood et al. 2009). Une étude multi-centrique qui a porté pendant un an sur des patients atteints de LGMDI a établi sans ambiguité la supériorité de l'imagerie quantitative eau-graisse. Celle-ci a détecté des variations statistiquement significatives du contenu graisseux de l'ordre de 1 à $4 \%$, alors que la gradation par l'échelle de Lamminen-Mercuri n'a mis en évidence aucun changement et l'évaluation fonctionnelle standard n'a montré qu'une tendance à la dégradation, sans atteindre le seuil de signification statistique, à l'exception des tests respiratoires (Willis et al. 2013).

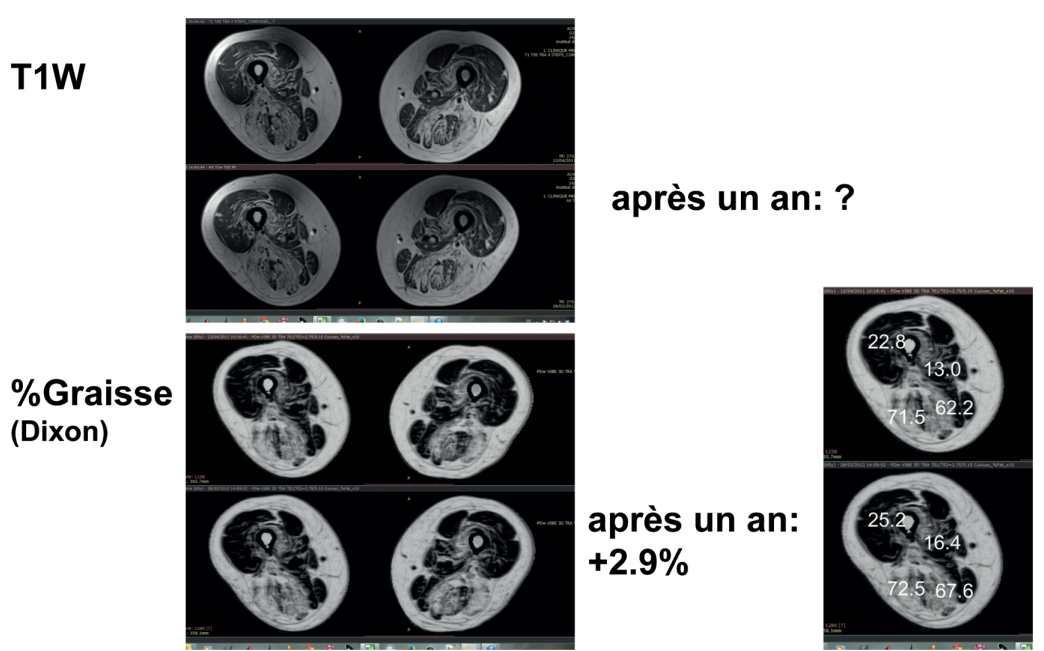

Figure 7

Évaluation qualitative versus quantification de la teneur en graisse musculaire. La similitude est très grande entre une imagerie pondérée en $\mathrm{T} 1$ (T1W) et l'image paramétrique du degré d'involution graisseuse calculée à partir d'acquisitions Dixon. Rien ne permet de les distinguer visuellement, tout comme la simple inspection d'images acquises un an plus tard chez le même patient (montrées en dessous de la première série) ne révèle aucun

changement. Dans l'image paramétrique, l'intensité du signal est une mesure du pourcentage de graisse. Et celui-ci augmente dans tous les muscles de la cuisse de ce patient atteint de maladie de Pompe, en moyenne de $2.9 \%$. Cet exemple illustre l'absolue nécessité de recourir à une imagerie quantitative pour suivre la progression de maladies.
Chez les patients atteints de dystrophie fascio-scapulo-humérale, l'imagerie quantitative eau-graisse a révélé une distribution bimodale particulière des lésions dégénératives au sein de leurs muscles, ainsi qu'une progression allant de distal en proximal pour les muscles affectés (Janssen et al. 2014).

Il a été montré chez les malades atteints de dystrophie musculaire oculo-pharyngée que le contenu en graisse des membres inférieurs pouvait augmenter jusqu'à $15 \%$ en 13 mois, alors qu'il restait inchangé chez les sujets contrôles d'âge équivalent (Fischmann et al. 2012). Ni l'évaluation fonctionnelle standard exhaustive (MFM), ni le score visuel des images ne détectait le moindre changement durant la même période d'observation.

Le pouvoir discriminant extrêmement élevé des méthodes Dixon a été confirmé chez des patients adultes atteints de la maladie de Pompe. Chez la plupart d'entre eux, les lésions dégénératives musculaires progressent lentement, à un rythme considérablement ralenti par rapport à celui observé dans les dystrophinopathies. Au niveau des membres inférieurs de ces patients, le taux annuel moyen d'infiltration graisseuse s'est révélé être inférieur à $1 \%$ mais a été détecté avec un haut degré de signification statistique (Carlier et al. 2015) (voir Figure 7).

Le suivi à un an de patients atteints de la maladie de Charcot-Marie-Tooth 1A a montré un accroissement significatif de la fraction graisseuse au niveau du mollet (1.2\%) mais pas au niveau de la cuisse $(0.2 \%)$ alors que chez les patients souffrant de myosite à inclusions la progression était de $2.6 \%$ au niveau des mollets et de $3.3 \%$ au niveau des cuisses (Morrow et al. 2015).

Chez les patients qui présentent des lésions de la coiffe des rotateurs, l'évaluation quantitative des changements dégénératifs graisseux a montré, plus que l'atrophie musculaire des muscles impliqués, une forte relation avec l'étendue des déchirures musculaires (Nardo et al. 2014; Nozaki et al. 2015).

Semblables observations ont également été faites dans des pathologies qui affectent secondairement le muscle strié squelettique.

Chez les patients atteints de sclérose latérale amyotrophique, une augmentation significative du T2 global des muscles des jambes a été notée au terme d'une période d'observation de 4 mois, signant la progression des dégénérescences graisseuses. Le déclin de la contraction isométrique volontaire maximale lors d'une dorsiflexion du pied était corrélée à celle-ci (Bryan et al. 1998).

Alors que le contenu total en graisse des muscles de la jambe n'était pas affecté, les diabétiques de type 2 
présentaient une distribution préférentielle des lipides en intramusculaire (Karampinos et al. 2012). L'imagerie Dixon corps entier a identifié un contenu musculaire en graisse accru en cas de paralysie périodique hyperkaliémique (Lee et al. 2015).

Des changements dans la composition des muscles striés squelettiques ont été systématiquement et régulièrement constatés chez les sujets âgés, même s'ils restent d'une amplitude limitée jusqu'à un âge très avancé. Le pourcentage du signal lipidique intramusculaire double généralement, de 2 à $4 \%$, entre la deuxième et la septième décennie (Schwenzer et al. 2009a; Alizai etal. 2012; Csapo et al. 2014 ; Morrow etal. 2014; Azzabou et al. 2015a). Pour l'essentiel, cette augmentation représente un véritable accroissement de la teneur lipidique du muscle et elle ne reflète qu'accessoirement la perte de tissu contractile avec l'âge (Csapo et al. 2014).

Le taux de graisse dans un muscle malade peut être considéré comme le reflet des dommages subis par le tissu tout au long de la vie du patient, ce qui en fait un biomarqueur fiable de la sévérité de la pathologie. Il parait dès lors légitime d'utiliser l'augmentation ou la stabilisation des infiltrations graisseuses musculaires sur une période définie comme un indice quantitatif de la progression de la maladie ou de la réponse au traitement. Dans la dystrophie musculaire, et plus particulièrement chez les garçons atteints de la maladie de Duchenne, la sévérité de la maladie peut être extrêmement variable d'un patient à l'autre, ce qui se traduit par des taux annuels de transformation de graisse variant de 3 à $15 \%$ (Fischmann et al. 2013; Wary et al. 2015b; Bonati et al. 2015a). Ceci complique considérablement l'interprétation des résultats d'une intervention thérapeutique. Si une augmentation de $5 \%$ de la graisse est mesurée sur une année de traitement, cela doit-il être interprété comme une réponse positive chez un patient sévèrement atteint ou comme une réponse négative chez un patient moyennement affecté ? À l'échelle d'un groupe de patients, on peut $y$ répondre en organisant un essai contre placebo. Cette approche classique ne résout pas la question de l'efficacité du traitement à l'échelon de l'individu et pose le problème éthique de retarder un traitement potentiellement efficace chez des patients souffrant d'une maladie mortelle. On pourrait également proposer d'utiliser chaque patient comme son propre contrôle et, après une période d'observation, déterminer si la pente de la progression du remplacement graisseux diminue après l'initiation du traitement. Cela permettrait d'apprécier la réponse au traitement pour chaque patient mais posera le même problème éthique. Parce que la teneur en graisse intramusculaire est un intégrateur naturel de tous les dommages subis par le muscle durant la vie du patient, il constitue par lui-même un puissant indicateur de la sévérité de la maladie à la condition de prendre en compte l'âge des sujets. À un âge donné, plus élevé est le contenu musculaire en graisse, plus important sera le taux de transformation graisseuse attendu au cours des mois à venir. Bien qu'elles soient en nombre limité, les données récoltées sur l'avant-bras au cours de l'étude de l'histoire naturelle Duchenne sponsorisée par le Généthon supportent cette assertion (Wary et al. 2015a). Des tables de référence pourraient être constituées en colligeant les données générées dans les différentes études en cours.

Dans le même ordre d'idée, une forte corrélation a été observée entre la teneur en graisse des différents muscles de la cuisse et de la jambe et le taux annuel de transformation graisseuse au sein de ces mêmes muscles dans un échantillon de patients adultes atteints de la maladie de Pompe. En effet, chez ceux-ci, la progression des dégâts musculaires est lente et relativement homogène entre individus, mais avec une grande variabilité de muscle à muscle (Carlier et al. 2015).

La relation entre la fraction de la graisse et sa progression tendra inévitablement vers un plateau aux valeurs élevées de contenu en graisse. On ne peut pas attendre que la fraction de graisse augmente de $15 \%$ quand le remplacement du muscle par la graisse a atteint $80 \%$, même si cela avait été le cas quand il n'était seulement que de $40 \%$. Une solution simple pour contourner la relation sigmoïdale entre la fraction de graisse et sa progression, serait de rapporter la progression du remplacement par la graisse à la fraction de tissu contractile résiduel, c'est-à-dire le véritable taux de transformation du muscle en graisse. En prenant l'exemple ci-dessus, les $15 \%$ d'augmentation de la graisse quand la masse de tissu contractile était encore de $60 \%$, équivaudrait, si la sévérité de la maladie reste constante, à une augmentation de $5 \%$ de la teneur en graisse si la fraction de tissu contractile est tombée à $20 \%$.

\section{"L'activité " de la maladie}

Des études sur des modèles murins de dystrophie musculaire ont montré il y a déjà plus de vingt ans que les tissus musculaires pathologiques présentaient des T2 élevés (McIntosh et al. 1998 ; Tardif-de Géry et al. 2000). La même observation a été réalisée chez le GRMD, un modèle canin de la myopathie de Duchenne, plus proche du phénotype humain (Thibaud et al. 2007 ; Thibaud et al. 2012 ; Wang et al. 2013) (voir Figure 8). De façon très intéressante, le T2 musculaire a tendance à se 


\section{muscles normaux}

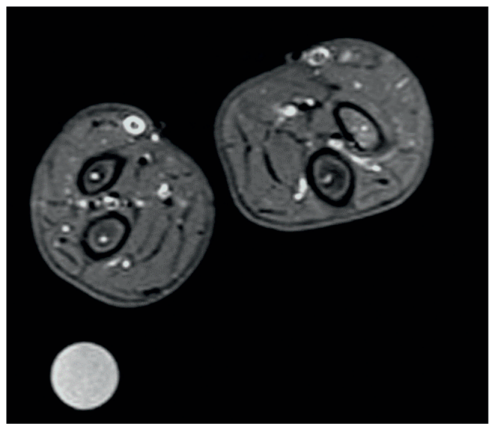

muscles dystrophiques

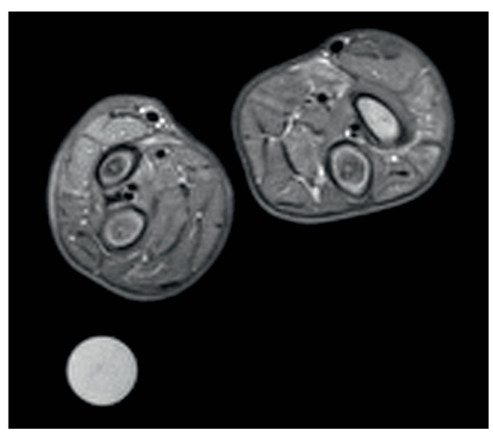

Figure 8

Imagerie pondérée en T2 montrant l'aspect caractéristique d'un muscle dystrophique, en l'absence d'infiltration graisseuse comme c'est le cas chez le chien GRMD. L'augmentation du T2 de l'eau musculaire est globale, mais avec des variations de muscle à muscle et avec également un aspect inhomogène des muscles les plus atteints.

normaliser lorsqu'une thérapie génique restaure efficacement la protéine défective (Walter et al. 2005 ; Pacak et al. 2007 ; Yokota et al. 2009).

Plus récemment, plusieurs travaux ont confirmé ces observations, et amélioré notre compréhension des processus d'altération du T2 sur ces modèles animaux de dystrophie musculaire. L'évolution temporelle du T2 musculaire a été précisément décrite chez la souris mdx. Un pic d'élévation du T2 est systématiquement observé entre 4 et 8 mois, suivi d'une baisse progressive (Pratt et al. 2013 ; Heier et al. 2014 ; Vohra et al. 2015c). Des anomalies du T2 ont également été décrites dans d'autres modèles de dystrophie (Vohra et al. 2015a ; Martins-Bach et al. 2015), avec des différences notables dans leur distribution à l'intérieur des muscles impliqués, notamment entre les souris Large et les souris mdx. Les muscles dystrophiques sont aussi caractérisés par une sensibilité plus importante à l'exercice excentrique. Cela a été illustré par une élévation anormalement importante du T2 de la souris mdx soumise à un exercice de course en descente (Mathur et al. 2011).

Des mesures quantitatives du T2 ont également été réalisées au cours de différents essais thérapeutiques sur ces modèles animaux. Après expression du gène de la micro-dystrophine chez la souris mdx, l'imagerie quantifiée du T2 a démontré un pouvoir discriminant supérieur à celui de l'imagerie de transfert d'aimantation et de l'imagerie du tenseur de diffusion (Park et al. 2015). Un autre essai, par saut d'exon U7, mené avec succès chez le chien GRMD a été accompagné d'une baisse significative du T2 dans les membres traités (Le Guiner et al. 2014). Enfin, un traitement au losartan a démontré un effet de normalisation du T2 musculaire sur un modèle murin de dystrophie congénitale lié au gène de la laminine (Vohra et al. 2015a).

Le T2, ou temps de relaxation spin-spin des molécules d'eau du muscle squelettique, peut être interprété comme un indicateur de l'activité de la maladie. Ce terme est intentionnellement vague étant donné qu'une variation de T2 est un processus non spécifique qui peut être engendré par différents mécanismes tels que l'inflammation, la nécrose, la dystrophie musculaire, la dénervation aiguë, ou toute autre situation pouvant être accompagnée par un œdème intracellulaire, extracellulaire, ou mixte. Cela a été montré de façon extensive sur des modèles animaux (Wishnia et al. 2001 ; Heemskerk etal. 2007; Bryant et al. 2014 ; Ha et al. 2015) ainsi que chez l'homme. L'exercice physique d'intensité modérée a beaucoup augmenté le T2 musculaire par un mécanisme d'accumulation d'eau dans les myocytes impliqués. Ce processus est transitoire, et contrairement à une élévation pathologique du T2, les valeurs de T2 se normalisent au bout de quelques heures. On comprend donc que les résultats d'une imagerie réalisée peu après un exercice physique pourront être faussés à cause de ce phénomène. C'est pour cette raison que nous conseillons de toujours réaliser l'examen radiologique avant les évaluations fonctionnelle, particulièrement chez les patients présentant des muscles fragilisés par la pathologie.

Comme déjà mentionné, les modèles pathologiques animaux ne développent que très rarement des infiltrations graisseuses au niveau des tissus musculaires. De ce fait, une augmentation globale de T2 mesurée par un ajustement mono-exponentiel de la décroissance du signal RMN sera interprétée de manière non ambiguë par une augmentation du T2 de l'eau musculaire. Chez l'homme, par contre, une'infiltration graisseuse est très fréquemment observée chez des patients atteints de pathologies neuro-musculaires chroniques. Les protons des lipides ayant des temps de relaxation T2 beaucoup plus élevés que les protons de l'eau, la présence de graisse dans les tissus musculaires, même à hauteur de quelques pourcents, augmentera de façon significative le T2 global mesuré avec un modèle monoexponentiel, vers des valeurs comparables à celles observées pour des tissus musculaires enflammés ou endommagés.

De nombreuses équipes ont mesuré le T2 des muscles infiltrés de graisse des patients Duchenne. Certaines ont observé une augmentation du T2 global avec l'âge des patients (Garrood et al. 2009; Kim et al. 2010 ; Arpan et al. 2013 ; Forbes et al. 2014 ; Willcocks et al. 2014 ; Kim et al. 2015), alors que d'autres, au contraire, ont observé la même diminution du T2 musculaire au cours du 
Tableau 1

Différences entre une mesure globale du T2 musculaire et celle du T2 de l'eau musculaire temps que celle décrite dans les modèles animaux (Forbes et al. 2014 ; Wary et al. 2015b). Ces résultats, apparemment contradictoires, ont de quoi dérouter le lecteur. L'explication réside dans le fait que les premiers ont déterminé le T2 global, son augmentation reflétant alors principalement les changements dégénératifs liés aux infiltrations graisseuses. Cette information est donc redondante avec celle fournie par les séquences de type Dixon, les résultats obtenus avec ces deux techniques étant fortement corrélés (Azzabou et al. 2015c). Les seconds ont mesuré spécifiquement le T2 de l'eau musculaire. La diminution progressive du T2 observée pourrait soit être due à la croissance, comme cela a déjà été montré chez des individus sains, au moins chez le chien (Thibaud et al. 2012), ou alors à l'épuisement progressif des capacités régénératives du muscle dystrophique.

\begin{tabular}{|l|l|l|}
\hline Mesure le signal de & \multicolumn{1}{|c|}{ T2 musculaire " global " } & \multicolumn{1}{c|}{ T2 de l'eau musculaire } \\
\hline Renseigne sur & l'eau et la graisse & de l'eau exclusivement \\
\hline Sensible aux & $\begin{array}{l}\text { essentiellement les lésions dégénératives } \\
\text { chroniques de nature graisseuse }\end{array}$ & l'"activité de la maladie " \\
\hline Considérations méthodologiques & changements lents, à long terme & changements rapides, à court terme \\
\hline
\end{tabular}

Par souci de clarté, nous préconisons l'emploi d'une terminologie précise : le T2 global, dont les altérations reflètent principalement les changements dégénératifs liés à l'infiltration graisseuse et le T2 de l'eau musculaire qui évalue plus spécifiquement l'implication du tissu musculaire à proprement parler dans le processus pathologique (Carlier 2014) (voir Tableau 1). Nous recommandons (c'est un point de vue personnel des auteurs) d'abandonner l'utilisation de l'évaluation du T2 global dans des muscles infiltrés de graisse car les résultats générés ne permettent pas de séparer clairement les deux processus pathologiques évoqués alors qu'ils peuvent l'être en utilisant d'autres méthodes. Cela génère, en outre, une ambiguïté qui peut être levée par l'utilisation de méthodes d'analyse plus sophistiquées pour l'étude de la décroissance T2 du muscle (voir ci-dessous, le paragraphe sur les développements méthodologiques récents).

L'utilisation du T2 de l'eau musculaire comme indicateur d'activité de la maladie est non seulement possible chez l'homme, elle constitue une variable pertinente, au moins autant que dans les modèles animaux. Le T2 de l'eau est anormalement élevé dans les muscles des enfants Duchenne (Arpan et al. 2013 ; Forbes et al. 2014 ; Wary et al. 2015b; Wokke et al. 2015), mais pas chez les patients Becker (Wokke et al. 2016). Chez les patients atteints de la forme tardive de la maladie de Pompe, environ un tiers des muscles examinés présentent une augmentation modérée du T2 de l'eau (Carlier et al. 2015). Dans les myopathies inflammatoires, le T2 de l'eau musculaire est systématiquement augmenté (Park et al. 1990 ; Maillard et al. 2004 ; Yao et Gai 2012). En imagerie diagnostique, l'inflammation liée à la pathologie est généralement détectée grâce à des séquences pondérées en T2 combinées avec une suppression de graisse (STIR) (Walker 2008; Degardin et al. 2010). Chez les patients atteints de dystrophie musculaire fascio-scapulo-humérale (DMFSH), certains muscles sont STIR positifs et comportent des signes inflammatoires à la biopsie (Tasca et al. 2012). Néanmoins, cette évaluation qualitative permet uniquement de détecter des contrastes entre des muscles sains et muscles altérés. Si tous les muscles d'un membre sont atteints, cet examen standard ne détectera aucune anomalie. Une telle situation s'est par exemple présentée chez des patients atteints de dermatomyosite juvénile (Carlier et al. 2013). Pour éviter ces faux-négatifs, nous préconisons d'utiliser systématiquement la quantification du T2 de l'eau pour l'évaluation et la réponse aux traitements des myopathies inflammatoires, même si cette solution n'est pas encore largement acceptée par la communauté médicale.

Outre le manque de spécificité du T2, il reste également difficile d'établir avec certitude des liens temporels entre les événements pathologiques et ces modifications de T2. Existe-t-il un délai entre les deux et si oui, quelle est sa durée? Par exemple, chez certains patients atteints de myopathies inflammatoires, il peut $y$ avoir discordance entre les mesures de T2 et le statut clinique. La fluctuation naturelle du T2 au cours des dystrophies musculaires n'est encore que très peu documentée.

De manière intéressante, il a été montré que le T2 de l'eau musculaire pouvait être élevé dans certaines myopathies congénitales, tout au moins dans des modèles animaux avec des mutations du gène de la 
nébuline, ACTA1 ou encore de la dynamine (Gineste etal. 2013b; Gineste et al. 2013c; Gineste et al. 2013a; Martins Bach et al. 2015). Cela tend à démontrer qu'un certain degré de désorganisation cellulaire peut altérer suffisamment les mouvements intracellulaires, et notamment ceux des molécules d'eau pour avoir un effet sur le T2. Le fait d'avoir observé une augmentation du T2 de l'eau chez des patients atteints de myopathies plus stable représente tout de même une limitation à l'utilisation généralisée de ce biomarqueur comme indicateur absolu de l'activité de la maladie. Dans le même ordre d'idées, les modifications du T2 de l'eau observées à la suite d'une dénervation sont dus à l'augmentation relative de l'espace extracellulaire qui accompagne l'atrophie du tissu musculaire (Polak et al. 1988; Fleckenstein et al. 1993; Kamath et al. 2008), ce qui reflète davantage une réorganisation structurelle du muscle que l'activité de la maladie au sens strict.

Il serait dès lors plus prudent de restreindre l'utilisation du T2 de l'eau musculaire au suivi de l'activité de la maladie dans des conditions maîtrisées, où l'on sait que les valeurs seront modifiées par des processus pathologiques destructifs, au moins pendant certaines périodes de leur évolution. Dans ces cas, la quantification du T2 de l'eau musculaire fournit indubitablement un biomarqueur représentatif de la progression de la pathologie ainsi que de la réponse à un traitement. De nombreuses études cliniques ont maintenant confirmé toutes les observations réalisées sur les modèles animaux. Il a été montré que la valeur du T2 avait une valeur prédictive quant à la vitesse de progression des altérations dégénératives chroniques. Chez les enfants Duchenne, l'instauration d'une corticothérapie est accompagnée rapidement d'une réduction de quelques $\mathrm{ms}$ du $\mathrm{T} 2$ au niveau des jambes, qui est suivie d'une stabilisation du processus d'infiltration graisseuse (Arpan et al. 2014). Comme mentionné précédemment, chez les patients adultes atteints de la maladie de Pompe, un tiers des muscles des membres inférieurs présente des altérations du T2 de l'eau, légères à modérées, sur au moins un des deux examens réalisés à un an d'intervalle. Dans ces muscles, le taux d'infiltration graisseuse a en moyenne augmenté $35 \%$ plus rapidement que dans les muscles présentant des T2 normaux (Carlier et al. 2015). Pour l'ensemble des muscles, cette étude a démontré une corrélation significative entre la valeur moyenne du $\mathrm{T} 2$ et le taux moyen d'infiltration graisseuse entre les deux visites. Chez les patients souffrant d'une DMFSH, les muscles qui présentaient des hyperintensités sur des images pondérées en T2 avec saturation de graisse, étaient ceux qui subissaient les plus fortes augmentations d'infiltrations graisseuses entre deux examens successifs (Friedman et al. 2013 ; Janssen et al. 2014). Ces résultats sont cruciaux et prouvent l'utilité du T2 de l'eau musculaire en tant que biomarqueur de l'activité de la maladie dans les pathologies neuromusculaires.

De nombreuses zones d'ombre restent cependant à éclaircir. Par exemple, quel sera le rôle du T2 de l'eau pour étudier la réponse à l'expression du gène de la dystrophine chez des patients Duchenne déjà sous traitement stéroïdien. Les stéroïdes normalisant quasiment les valeurs de T2, il n'est dès lors pas certain que l'expression partielle du gène de la dystrophine, dans des proportions identiques à celles obtenues dans les essais thérapeutiques de saut d'exon s'accompagnera d'une nouvelle baisse significative $\mathrm{du}$ T2. Des résultats préliminaires laissent à croire que si cette baisse existe, elle sera difficile à détecter (observations personnelles des auteurs).

Différents problèmes méthodologiques et expérimentaux rendent compliquée la détermination précise du T2. Nous avons déjà évoqué en détail les difficultés engendrées par l'infiltration et le remplacement graisseux des muscles. Il est extrêmement difficile d'obtenir une suppression parfaite du signal RMN des lipides, et leur présence, même à hauteur de quelques pourcents suffit déjà à perturber la mesure du T2 de l'eau. Les méthodes les plus efficaces à ce jour ne visent pas à minimiser ou éliminer totalement le signal de la graisse, mais plutôt à séparer, à l'acquisition ou à la reconstruction, les contributions de l'eau et des lipides en tirant profit du déplacement chimique entre les deux espèces de protons, ou encore de la différence entre leurs temps de relaxation T2. Ces méthodes sophistiquées ne sont pas nombreuses, et les plus populaires sont basées sur l'acquisition d'échos de spin multiples, en régime CPMG, couvrant une large gamme de temps d'échos. La séquence IDEALCPMG a été développée en ajoutant à cette séquence un schéma d'acquisition de type Dixon qui permet de séparer les contributions de l'eau et de la graisse (Janiczek et al. 2011). Une autre option consiste à réaliser un ajustement tri-exponentiel de la décroissance du signal obtenu par la séquence d'échos de spin multiples, et, à profiter de la différence importante entre les T2 de l'eau et de la graisse pour séparer avec une grande robustesse les deux composantes de protons (Azzabou et al. 2015c). Cette méthode de post-traitement a le grand avantage de reposer sur un schéma d'acquisition très classique, disponible sur la plupart des scanners RMN cliniques. Elle est par conséquent facilement implémentée dans un essai multicentrique, ce qui se vérifie de plus en plus. 
Pour être exacte, la mesure du T2 exige une méthodologie rigoureuse. Dans une séquence d'échos de spin multiples, les impulsions d'excitation et de refocalisation doivent être précisément ajustées à $90^{\circ}$ et $180^{\circ}$. Si les angles de bascule s'écartent de ces valeurs nominales, la décroissance du signal RMN va être trop rapide dans le cas où des gradients de déphasage efficaces sont appliqués (ce qui est rarement le cas sur des systèmes cliniques), ou trop lente, dans le cas contraire où des échos stimulés vont se former et s'additionner aux échos de spin (Hollingsworth et al. 2012). Une approche pratique consiste à acquérir, en plus de la séquence CPMG, une autre séquence pour cartographier le champ magnétique $\mathrm{B} 1$, et connaître exactement l'angle de bascule appliqué à chaque voxel du volume d'intérêt (Yarnykh 2007). Lorsque celui-ci s'écarte significativement de la valeur prescrite, le voxel en question est retiré de l'analyse T2 (Azzabou et al. 2015c). Cette méthode est simple et efficace, mais elle a le désavantage d'éliminer un grand nombre de voxels dans les zones où le $\mathrm{B} 1$ n'est pas homogène. D'autres solutions plus efficaces ont été proposées et sont sur le point d'être implémentées dans des protocoles de recherche clinique. Des modèles plus réalistes que de simples exponentielles peuvent être utilisés pour décrire précisément la formation des échos de spins et des échos stimulés, et permettent de s'affranchir de l'acquisition de la séquence de cartographie de B1. Par exemple, le formalisme EPG (pour Extended Phase Graphs), est un outil
T2 de l'eau
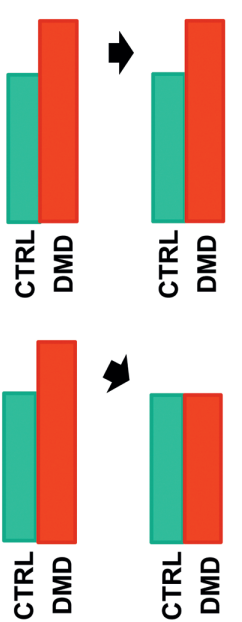

$\%$ graisse

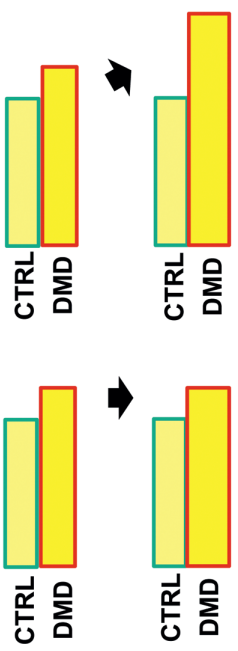

Figure 9

Résumé des informations complémentaires apportées par les deux principaux biomarqueurs, le T2 de l'eau musculaire et le \% de graisse musculaire. Si la maladie est active et stable, on attend que le T2 soit augmenté et également stable au cours du temps alors que l'involution graisseuse progressera. Si un traitement efficace est appliqué, il devrait logiquement ramener le T2 à une valeur normale et induire une stabilisation de la teneur en graisse. puissant permettant de décrire et de comprendre le comportement de l'aimantation d'un voxel avec des temps de relaxation $\mathrm{T} 1$ et $\mathrm{T} 2$, soumis à une série d'impulsions radiofréquence et de gradients arbitraires. Lebel et Wilman ont été les premiers à modéliser le signal obtenu par la séquence MSME avec le formalisme EPG afin d'estimer précisément le T2 dans des zones présentant des échos stimulés (Lebel et Wilman 2010). Il a depuis été montré que cette méthode pouvait être utilisée pour améliorer la précision et la concordance des mesures de T2 musculaires entre plusieurs sites (Rooney et al. 2011).

Plus que la précision, ce qui compte en recherche clinique, c'est le pouvoir discriminant de la technique évaluée. Est rassurant en ce qui concerne les mesures de T2 de l'eau, le fait que, même si la mesure est inexacte à cause de la perturbation induite par les échos stimulés, celle-ci reste sensible aux effets pathologiques et à la réponse aux traitements. Ainsi, une étude clinique a suivi par quantification du T2 musculaire les effets de l'inflammation et sa correction par une corticothérapie même si les mesures étaient supérieures d'un facteur 2 à ce qu'elles auraient dû être à l'aide d'une méthodologie optimisée (Maillard et al. 2004).

\section{Les relations entre les outils d'évaluation fonctionnelle et la RMN}

Une des questions les plus fréquemment posées concerne la relation entre les outils d'évaluation fonctionnelle standards et les ceux dérivés de l'imagerie. Lorsqu'un clinicien-chercheur est à court d'imagination, une question récurrente fuse : dans quelle mesure les outils RMN corrèlent-ils avec la fonction musculaire? La réponse est connue par avance. Oui, il y a une corrélation significative entre les deux classes d'outils d'évaluation, et le coefficient de corrélation est généralement aux alentours de 0.7. Il est en effet évident que la performance du muscle va être fortement liée à sa masse et à sa composition. Cela avait clairement été démontré dès les premières études réalisées sur des patients Duchenne avec de simples indices qualitatifs de l'évaluation de l'infiltration graisseuse (Liu et al. 1993), et systématiquement confirmé depuis (Wren et al. 2008; Kim et al. 2010; Torriani et al. 2012 ; Gaeta et al. 2012 ; Fischmann et al. 2013; Wokke et al. 2014b ; Vohra et al. 2015b). Des études récentes l'ont également démontré pour d'autres pathologies: la dystrophie myotonique de type I (Hiba et al. 2012), la maladie de CharcotMarie-Tooth, la myosite à inclusions (Morrow et al. 2015), mais également pour les lésions de la coiffe des rotateurs (Nardo et al. 2014), et le vieillissement (Csapo et al. 2014). Très logiquement, la force 


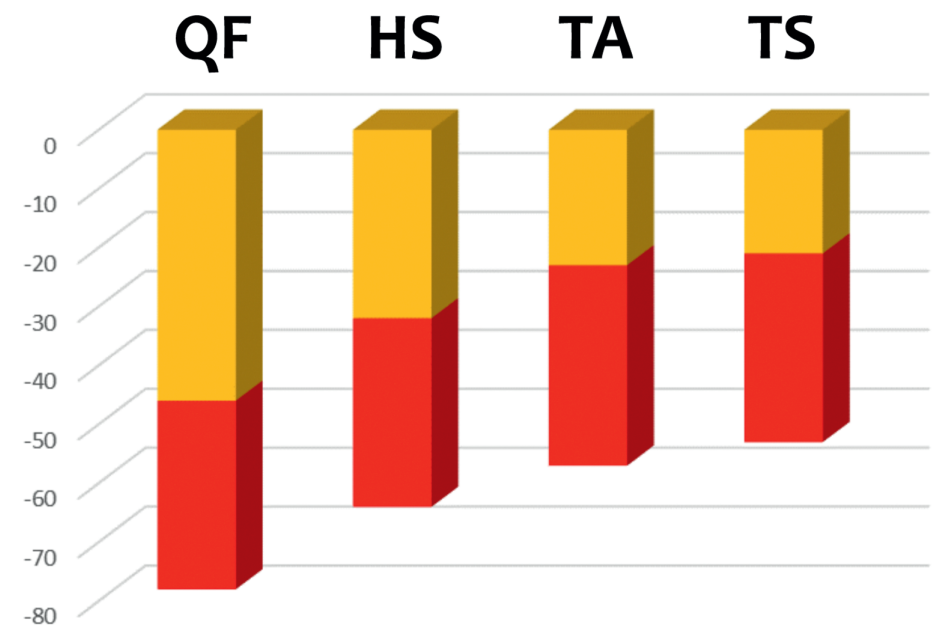

\section{\% de diminution de la force musculaire maximale destruction musculaire altérations de contractilité}

Figure 10

Diminution de la force musculaire maximale des patients Duchenne, exprimée en \% des valeurs mesurées chez des enfants normaux de même âge. En orange, la part attribuable à la destruction du tissu musculaire, en rouge, la part attribuable aux altérations des propriétés contractiles du muscle. QF : quadriceps femoris, HS : ischio-jambiers, TA : tibialis anterior, TS : triceps suralis. Modifié d'après Wokke et al., $2014 \mathrm{~b}$.

musculaire corrèle mieux avec la masse de tissu musculaire résiduelle (Wokke et al. 2014b) qu'avec le pourcentage d'infiltration graisseuse (Wren et al. 2008).

De nombreuses situations démontrent la complémentarité entre les deux modalités. La mesure de l'aire transversale contractile permet par exemple de déterminer si une diminution de la force ou du couple de force est due à la perte musculaire ellemême ou si elle est plutôt liée à une altération de la qualité de la contraction musculaire. Chez les enfants Duchenne, la force musculaire, lorsqu'elle est normalisée à la masse contractile résiduelle, reste anormalement faible, ce qui sous-tend l'existence d'anomalies contractiles du tissu musculaire dystrophique (Wokke et al. 2014b ; Vohra et al. 2015b) (voir Figure 10).

D'autres questions pertinentes peuvent être abordées. Quelle est la relation temporelle entre l'évolution de la composition musculaire et celle des altérations fonctionnelles? Des données provenant du consortium Imaging-DMD, présentées à la conférence WMS (Voit 2014) mais non encore publiées, montrent que le test de marche de 6 minutes, qui est l'outil d'évaluation standard dans les essais, ne se dégrade guère avant que les muscles des jambes des enfants Duchenne ne soient infiltrés à $60 \%$ par de la graisse. Cela suggère que les outils d'imagerie pourraient avoir un rôle prédictif de l'évolution clinique. Cette observation, potentiellement d'une importance majeure, doit être confirmée. D'autres études, également chez les patients atteints de la maladie de Duchenne indiquent que le déclin de la force musculaire se produit en parallèle avec la progression des changements dégénératifs (Wokke et al. 2014b; Vohra et al. 2015b), ou pourrait même les précéder au niveau des avant-bras (Hogrel et al., 2016).

Une autre question majeure est le pouvoir discriminant des outils d'évaluations. Comment comparer les biomarqueurs quant à leur capacité à détecter les effets d'une intervention, quel est le plus petit changement qui peut être mis en évidence de manière statistiquement significative dans le plus petit échantillon de population? Parce qu'ils sont largement sinon totalement indépendants de la collaboration du sujet, la plupart des indices quantitatifs de l'imagerie par RMN offrent la meilleure reproductibilité et ont été proposés comme les meilleurs candidats pour détecter et évaluer les effets d'une thérapie (Hiba et al. 2012 ; Bonati et al. 2015a). Cette affirmation est basée sur l'hypothèse qu'un agent thérapeutique induira des changements d'amplitude identique pour toutes les mesures d'évaluation, ce qui est peu probable. La question reste ouverte mais, la moindre dépendance, même si elle ne peut complètement s'en affranchir, de la RMN à la collaboration $\mathrm{du}$ patient constitue un atout majeur.

Notre enthousiasme doit être quelque peu tempéré par le scepticisme montré par la FDA pour l'utilisation de la RMN comme outil d'évaluation de la réponse du muscle dystrophique au saut d'exon 51 par le drisapersen. L'agence de régulation a émis des réserves sur l'impact de la diversité des plateformes d'imagerie, mais aussi à propos des différences entre les protocoles d'imagerie et entre les paramètres d'acquisition, ainsi que les procédures d'analyse des images sur les variables estimées de la RMN. Il y avait également des remarques sur les insuffisances des contrôles de qualité de la stabilité des systèmes et de l'évaluation de reproductibilité. Il a été reproché que l'effet attendu du traitement pourrait être du même ordre que la variabilité de la technique. La petite taille des échantillons de population a également été critiquée. Les incertitudes concernant la relation entre les variables de la RMN et les mesures fonctionnelles ont également été soulignées. Fait intéressant, il y avait à nouveau une certaine confusion dans ce rapport entre le T2 global du muscle et le T2 de l'eau du muscle. La demande de la FDA d'une harmonisation complète 
des procédures entre les sites est irréaliste d'un point de vue technique. La seule façon de rassurer les agences de régulation sur la valeur des mesures par $\mathrm{RMN}$ sera de valider minutieusement les processus qui génèrent les images paramétriques et de démontrer l'indépendance des résultats par rapport à des conditions d'acquisitions légèrement différentes.

Ce qui apporterait un avantage définitif à la RMN serait l'identification de biomarqueurs qui seraient capables de prédire très précocement l'évolution clinique ultérieure du patient, avant toute modification fonctionnelle ou anatomique. En oncologie, des indices spectroscopiques RMN sont utilisés pour détecter très précocement les réponses positives au traitement avant tout signe morphologique de régression de la tumeur (Redmond et al. 1992 ; Shin et al. 2012 ; Leong et al. 2015). Des marqueurs similaires doivent encore être identifiés pour les maladies neuromusculaires. La concentration en phospho-diesters musculaires est un candidat potentiel en cas de dystrophie musculaire où leur concentration est élevée en relation avec l'augmentation du turnover des phospholipides membranaires (Edwards et al. 1982 ; Kemp et al. 1993 ; Wokke et al. 2014a ; Wary et al. 2015a), et semble se normaliser très rapidement quand la dystrophine est exprimée (Le Guiner et al. 2014).

Il y a donc de multiples raisons de poursuivre la confrontation entre les biomarqueurs fonctionnels et l'imagerie chez les patients atteints de maladies musculaires. Comme règle de conduite générale, ce sont les écarts et les déviations aux corrélations strictes qui doivent susciter de l'intérêt. Démêler les mécanismes qui sont à leur origine fera avancer les connaissances alors que la contemplation d'un accord parfait est rassurante mais reste intrinsèquement stérile.

\section{Le rôle de la spectroscopie RMN du ${ }^{31} \mathbf{P}$ comme outil d'évaluation}

La spectroscopie RMN in vivo s'est avérée être un outil extrêmement puissant pour l'exploration atraumatique du métabolisme énergétique musculaire, au moyen des noyaux ${ }^{1} \mathrm{H},{ }^{31} \mathrm{P},{ }^{13} \mathrm{C}$ pour ne nommer que les plus importants (Boesch 2007). La spectroscopie du RMN du ${ }^{31} \mathrm{P}$ identifie et quantifie des molécules-clés du métabolisme énergétique telles que la phosphocréatine, l'ATP et le phosphate inorganique (Kemp et al. 2007). Elle mesure le $\mathrm{pH}$ intracellulaire. La concentration d'ADP libre cytosolique ainsi que la production d'ATP par les voies oxydatives et non-oxydatives peuvent être déterminées. Conjuguée à la mise en ceuvre relativement simple d'antennes de surface posées directement en regard du segment d'intérêt, la spectroscopie du ${ }^{31} \mathrm{P}$ a contribué énormément à l'avancée des connaissances sur l'énergétique musculaire, sa régulation à l'exercice et les perturbations introduites par les pathologies (Chance et al. 1986; Heerschap et al. 1999; Argov et al. 2000). À ce jour, la spectroscopie RMN n'a toutefois pas joué un role significatif comme outil d'évaluation des maladies neuromusculaires.

Des anomalies remarquables du spectre ${ }^{31} \mathrm{P}$ des enfants Duchenne ont été décrites plusieurs années avant que la dystrophine ne soit identifiée et que son implication dans la pathogenèse de la dystrophie musculaire de Duchenne ne soit reconnue (Edwards et al. 1982 ; Newman et al. 1982 ; Chance et al. 1986). Il est intéressant de noter que ces anomalies sont présentes au repos, ce qui simplifie considérablement leur détection. Elles révèlent des anomalies qui existent à différents niveaux sub-cellulaires; la mitochondrie, l'appareil contractile et surtout la membrane sarcoplasmique (Younkin et al. 1987 ; Kemp et al. 1993 ; Tosetti et al. 2011 ; Wary et al. 2015a): la quantité totale des composés phosphorés est abaissée, conséquence de l'atrophie musculaire et de l'adiposité ; le rapport phosphocréatine/ATP est également plus bas, reflétant la perte de tissu contractile, le rapport phosphate inorganique/phosphocréatine est anormalement élevé, révélateur de taux d'ADP au repos augmenté. Ces derniers traduisent une dysrégulation du contrôle des oxydations phosphorylantes mitochondriales et/ou une sollicitation énergétique accrue pour tenter de maintenir l'homéostasie ionique en dépit d'une perméabilité membranaire anormale. Le $\mathrm{pH}$ mesuré est alcalin, ou plus précisément, il y a un pool prééminent de phosphate inorganique avec un $\mathrm{pH}$ plus élevé, pool qui provient de cellules dystrophiques endommagées et/ou d'une expansion du compartiment interstitiel associée à la fibrose (Wary et al. 2012) ; et, enfin, la résonance dite des phosphodiesters, provenant essentiellement de la glycérophosphocholine, est élevée, un indicateur d'un turnover accéléré des phospholipides membranaires dans le myocyte dystrophique (voir Figure 11).

Il semble qu'aucune exploration dynamique de l'énergétique musculaire n'ait été jusqu'à présent pratiquée sur des garçons atteints de la maladie de Duchenne. Chez les patients Becker et les porteuses $\mathrm{DMD} / \mathrm{BMD}$, des altérations de la régulation du $\mathrm{pH}$ ont été décrites, pendant et après exercice, sans toutefois pointer systématiquement dans la même direction (Barbiroli et al. 1992a; Barbiroli et al. 1992b ; Barbiroli et al. 1993 ; Kemp et al. 1993 ; Tosetti et al. 2011). L'activité glycolytique a été mesurée à la hausse comme à la baisse, alors que les oxydations phosphorylantes étaient le plus 

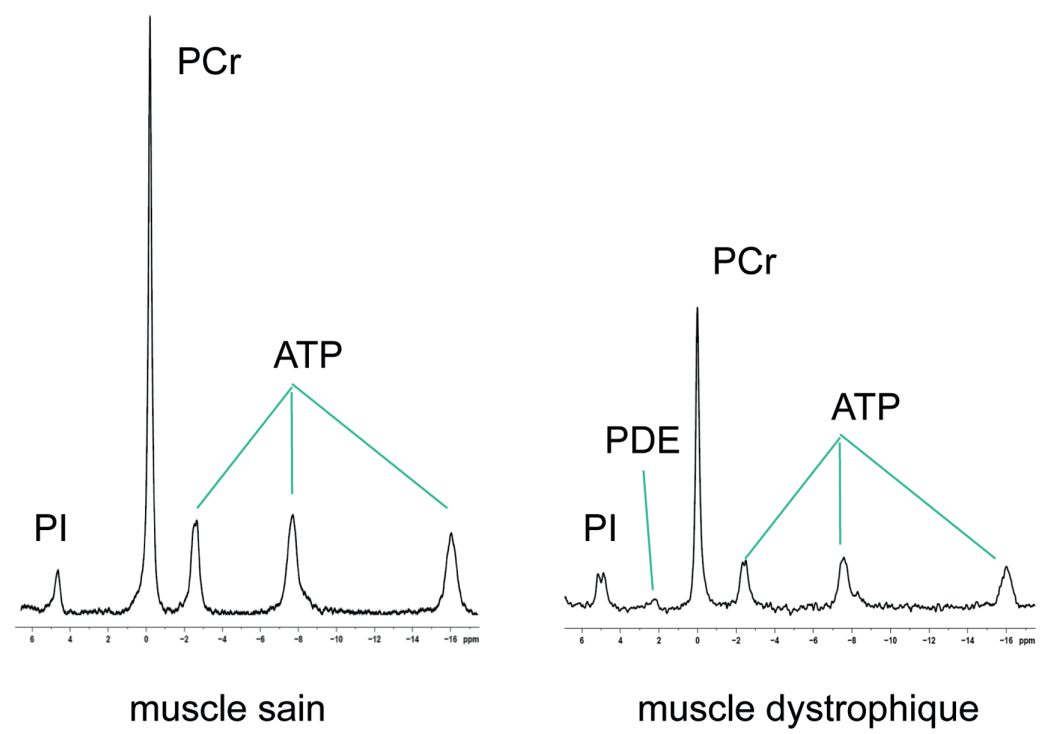

Figure 11

Exemples de spectres $\mathrm{RMN}^{31} \mathrm{P}$ musculaires montrant les anomalies patentes d'un muscle dystrophique en comparaison d'un muscle normal. Ces anomalies sont visibles dans le muscle à l'état de repos. Voir description dans le texte. Spectre pris chez le chien GRMD les mêmes altérations sont présentes dans le muscle de patients Duchenne. $\mathrm{PCr}$ : phosphocréatine, PI : phosphate inorganique, PDE : phospho-diesters.

souvent normales (Lodi et al. 1999 ; Kemp et al. 1993 ; Tosetti et al. 2011; Torriani et al. 2012). Globalement, les résultats des mesures à l'exercice sont considérablement plus dispersés et ne montrent pas le caractère systématique observé au repos.

L'étendue des perturbations du spectre ${ }^{31} \mathrm{P}$ au repos corrèle à la gravité de la maladie, étant déjà détectables chez les porteuses Duchenne, plus marquées chez les patients Becker et évidentes chez les garçons Duchenne (Kemp et al. 1993). Les anomalies spectrales observées dans les muscles fléchisseurs des doigts de patients atteints de la maladie de Duchenne sont plus sévères chez les patients nonambulants que chez les ambulants, et sont corrélées au nombre d'années passées en fauteuil (Wary et al. 2015a). Chez les patients atteints de DMFSH, le rapport $\mathrm{PCr} / \mathrm{ATP}$ s'effondre dans le même temps que le muscle entre dans une phase de destruction rapide et de remplacement graisseux (Janssen et al. 2014).

Toujours au niveau des fléchisseurs des doigts de patients atteints de la maladie de Duchenne, la progression de ces anomalies a été monitorée pendant une année; elle a été significative chez les patients non-ambulants (Hogrel et al. 2016). Chez le chien GRMD, les antérieurs traités par un exon-skipping U7 introduit par virus associé à l'adénovirus, ont vu leurs taux élevés de phospho-diesters revenir à des valeurs normales (Le Guiner et al. 2014). Si elles restent relativement anecdotiques, ces observations suggèrent fortement que la spectroscopie $\mathrm{du}^{31} \mathrm{P}$, et en particulier la résonance des phospho-diesters, puisse fournir des biomarqueurs utiles pour suivre le réponse du muscle dystrophique à l'expression de dystrophine. La spectroscopie du ${ }^{31} \mathrm{P}$ évalue l'intégrité et la stabilité de la membrane sarcoplasmique, ce qui lui confère un certain degré de spécificité et complète l'information plus globale donnée par les cartographies de la fraction de graisse et le T2. Il est intéressant de signaler qu'il a été récemment rapporté que le rapport PDE/ATP est anormalement élevé dans les muscles de patients atteints de la dystrophie musculaire de Becker à un stade où aucune élévation significative du taux d'infiltration graisseuse n'est encore détectable (Wokke et al. 2014a). Ceci suggère que cet indice puisse être un biomarqueur précoce du processus dystrophique, alors que le T2 de l'eau ne semble pas discriminant sur ce même échantillon de population (Wokke et al. 2016).

\section{Outils d'évaluation par imagerie RMN actuellement en cours de développement}

Les développements en cours sont orientés dans deux directions principales :

- l'identification de variables RMN qui reflètent d'importantes modifications histologiques, physiologiques ou biochimiques, qui ne peuvent être détectées par les approches d'imagerie standard actuelle (p. ex. la fibrose ou les troubles micro-circulatoires), - l'utilisation de contrastes RMN, déjà connus, voire originaux, aux fins d'étendre et d'améliorer la caractérisation du muscle squelettique pathologique (p. ex. des mesures de T1 ou de diffusion).

\section{- La fibrose}

Le remplacement du muscle squelettique strié par du tissu conjonctif, majoritairement du collagène, constitue l'autre forme de transformation dégénérative chronique qui affecte les tissus musculaires pathologiques (Wynn 2008; Klingler et al. 2012; Kharraz et al. 2014). Plus encore que l'infiltration graisseuse, dont les effets délétères sont indirects et liés à la perte de tissu contractile associée, la fibrose impacte directement la fonction musculaire. La fibrose endomysiale est fortement corrélée à la perte de fonction motrice chez les patients atteints de la maladie de Duchenne (Desguerre et al. 2009). C'est pourquoi un pan de la recherche thérapeutique vise à bloquer voire à rendre réversible l'accumulation pathologique de tissu conjonctif (Zhou et Lu 2010). Si l'imagerie pouvait amener des biomarqueurs de la fibrose, ceux-ci constitueraient une aide cruciale à l'évaluation de l'efficacité de ces agents anti-fibrotiques. Malheureusement, si la détection et la quantification des infiltrations graisseuses sont facilement 
accessibles par des méthodes dédiées d'imagerie RMN, l'évaluation de la fibrose interstitielle par $\mathrm{RMN}$ reste jusqu'à présent un défi majeur à relever. Le grand nombre de méthodes RMN qui ont été, et sont encore, proposées afin de détecter la fibrose reflète avant tout le fait qu'aucune d'entre elles ne donne pour le moment entière satisfaction.

Le collagène, les autres macromolécules de l'espace interstitiel, mais également leurs molécules d'eau d'hydratation ont des T2s très courts, de l'ordre de une à quelques centaines de $\mu \mathrm{s}$ (Edzes et Samulski 1978 ; Siu et al. 2015). Avec des séquences standards ou même dites "rapides ", acquises à des TE $\mathrm{de}$ une à quelques ms, le signal RMN de ces spins est totalement déphasé au moment de la réception et ne contribue plus à l'image. Les tissus conjonctifs denses, comme les tendons ou les tissus cicatriciels apparaissent comme des plages dépourvues de signal, et sont facilement détectables en imagerie standard. La fibrose interstitielle devrait également abaisser l'intensité du signal du voxel proportionnellement au volume qu'elle occupe. Néanmoins, sur ces images, les fluctuations dues à la fibrose qui devraient être typiquement de l'ordre de quelques pourcents sont masquées par les variations spatiales du signal RMN tissulaire induites par leur distance aux antennes de surface de réception. Les tentatives de correction de cette inhomogénéité de signal radiofréquence, bien que visuellement satisfaisantes, n'ont pour l'instant pas permis d'isoler et de quantifier la perte de signal générée par les dépôts de tissus conjonctifs. Ce phénomène peut tout de même contribuer aux distributions d'intensité de signal anormalement larges, observées dans des muscles profondément remodelés par une pathologie, la fibrose étant bien souvent un composant essentiel de ce remodelage. L'hétérogénéité du signal musculaire, en particulier sur les images pondérées en T2, est, selon nous, une caractéristique reproductible du muscle dystrophique et est très probablement en partie le reflet de la fibrose locale, dans une proportion difficile à quantifier pour le moment (Parzy et al. 2007 ; Thibaud et al. 2012 ; Wary et al. 2015a).

La décroissance T2 du signal des tissus est un processus multi-exponentiel complexe. Lorsque celle-ci est échantillonnée très finement sur des modèles animaux, il est possible de générer des spectres du T2 qui font apparaître des composantes à temps de relaxation très courts, qui ont été attribuées aux macromolécules présentes dans les tissus (Saab et al. 1999; Saab et al. 2000). Cette méthode a été transférée chez l'homme sous le nom d'ISISCPMG. Elle a permis d'identifier des composantes T2 intra- et extracellulaires mais n'a jusqu'à présent pas mis en évidence cette composante de spins à T2 très court (Araujo et al. 2014).

Les expériences de transfert d'aimantation sont bien adaptées à l'étude des échanges entre spins ; que ce soit à travers des interactions dipôle-dipôle, ou des échanges chimiques entre des groupes de spins mobiles ou semi-mobiles. En pratique, sur des tissus biologiques, elles permettent de mesurer les taux d'échange entre l'eau libre et les macromolécules, ainsi que les concentrations relatives de ces différents groupes de protons (Morrison et Henkelman 1995 ; Henkelman et al. 2001). Par le passé, de nombreuses études ont essayé d'établir un lien entre le contraste de transfert d'aimantation et la concentration en collagène tissulaire, en particulier au niveau du foie, mais n'ont généré que des résultats mitigés. Très récemment, des séquences d'imagerie rapide avec des schémas de saturation optimisés ont donné des résultats encourageants avec une bonne corrélation entre le contraste de transfert d'aimantation et le taux de fibrose dans le foie (Yarnykh et al. 2015). Le contraste de transfert d'aimantation est élevé dans le muscle squelettique et son origine et les mécanismes sous-jacents ont été étudiés par plusieurs groupes dans le muscle normal, vieillissant, et pathologique, avec une attention particulière sur son lien avec la fibrose, qui reste toujours insuffisamment compris (Schwenzer et al. 2009a; Sinclair et al. 2010 ; Sinclair et al. 2012 ; Morrow et al. 2014 ; Li et al. 2014 ; Li et al. 2015). La corrélation négative entre le contraste de transfert d'aimantation et l'âge dans une cohorte d'individus sains est par exemple, relativement déroutante (Morrow et al. 2014). Ces résultats ont cependant été obtenus pour une fréquence et une puissance d'irradiation particulière et ne peuvent donc pas être extrapolés facilement à d'autres conditions de saturation. L'effet négatif de l'infiltration graisseuse sur le contraste de transfert d'aimantation a également été évalué ( $\mathrm{Li}$ et al. 2015). Des expériences de transfert d'aimantation combinées à du filtrage double quantum et de l'imagerie à temps d'écho très court (UTE) ont également été réalisées dans le but d'améliorer la spécificité de cette technique au collagène (Kusmia et al. 2013 ; Eliav et al. 2014). Les résultats sont encourageants mais doivent pour le moment toujours être considérés comme préliminaires.

De quasi ignorée jusqu'à devenir la base d'une des méthodes les plus efficaces et populaires pour détecter la fibrose myocardique, une expansion de l'espace interstitiel accompagne le remplacement des myocytes par du tissu conjonctif. Cet accroissement du volume interstitiel peut être facilement quantifié après injection d'un agent de contraste à faible poids moléculaire à base de gadolinium $(\mathrm{Gd})$, 
comme le Gd-DTPA ou le Gd-DOTA qui va diffuser rapidement dans l'espace interstitiel où sa concentration va s'équilibrer avec celle du plasma. Quelques minutes après injection, au moment où la distribution de l'agent de contraste est en pseudo-équilibre, l'augmentation du taux de relaxation T1 dans le tissu myocardique normalisé à l'augmentation du taux de relaxation $\mathrm{T} 1$ dans le plasma permet d'estimer le pourcentage du volume extracellulaire du tissu (Miller et al. 2013; Moon et al. 2013). Bien qu'indirecte, cette technique s'est imposée comme la référence pour quantifier les dépôts pathologiques de tissu conjonctif dans le myocarde. Un prérequis à l'utilisation de cette méthode de quantification de fibrose est l'absence d'œedème extracellulaire ou d'anomalies cellulaires qui pourraient avoir pour conséquence une accumulation locale d'agent de contraste Gd ou sa pénétration dans les myocytes (p. ex. la nécrose, l'inflammation ou même une dystrophie). Dans ces cas-là, ou lorsque ces phénomènes sont suspectés d'avoir lieu en même temps que le remodelage structurel, le volume de distribution de l'agent de contraste $\mathrm{Gd}$ ne peut plus être utilisé comme un marqueur de substitution de la fibrose.

Ce même principe, et la méthodologie associée peuvent être utilisés pour détecter indirectement la fibrose interstitielle dans le muscle squelettique, mais cette technique n'a pas été évaluée jusqu'à présent. Certaines difficultés peuvent être attendues compte tenu du plus faible volume extracellulaire dans le muscle squelettique, mais aussi en relation avec le moindre degré de fibrose attendu dans le muscle pathologique, ainsi que du fait de l'absence d'un signal sanguin de référence facilement accessible comme c'est le cas au niveau cardiaque dans les cavités ventriculaires.

Pour ces mêmes raisons, et parce que la concentration en sodium est plus de 10 fois supérieure dans l'espace extracellulaire que dans les cellules, la concentration globale en sodium du myocarde est augmentée en cas de fibrose, et l'imagerie RMN du ${ }^{23} \mathrm{Na}$ peut être utilisée pour détecter ce phénomène. Néanmoins, cette évaluation est également indirecte et basée sur des changements non-spécifiques; les mêmes réserves que celles formulées dans le cas des agents de contraste à base de $\mathrm{Gd}$ s'appliquent dans le cas où la concentration en sodium dans les tissus augmente pour d'autres raisons, avec une liste de situations d'interprétation délicate identique à celle énoncée précédemment (Madelin et Regatte 2013). Le signal RMN peut être sensibilisé au mouvement à une échelle submillimétrique et cela peut servir à suivre la propagation des ondes de pression. La vitesse de propagation des ondes de pression augmente avec la rigidité du milieu traversé. Ce marqueur peut donc être utilisé pour estimer les propriétés viscoélastiques d'un tissu (Glaser et al. 2012). Ce phénomène est la base de l'élastographie par IRMN, qui est une méthode validée pour la quantification de la fibrose hépatique. Cette technique a été transposée au domaine du muscle squelettique et un certain nombre de situations ont été évaluées (Ringleb et al. 2007). Le muscle squelettique constitue un ensemble structurel hautement différencié, lui-même au sein de l'environnement complexe. Il ne semble dès lors pas trivial d'établir un lien direct entre la vitesse de propagation d'une onde de pression à une position donnée et la structure tissulaire du muscle en ce même point. L'environnement global des éléments contractiles et non-contractiles doit avoir un impact et contrôler la vitesse des ondes de pression en tout point du muscle. En particulier, les effets des dépôts de graisse sont susceptibles d'être non négligeables sur la vitesse de propagation des ondes de pression. Il en est de même pour les œdèmes et autres phénomènes inflammatoires (McCullough et al. 2011). Encore une fois, cette technique peut manquer de spécificité. Il est également à noter que les propriétés visco-élastiques des tissus peuvent également être évaluées par des méthodes ultrasonores, avec des résultats comparables à la RMN mais un net avantage en terme de facilité d'implémentation (Drakonaki et al. 2012).

Une détection plus directe de la fibrose a également été proposée. Elle repose sur l'utilisation de peptides courts marqués au Gd, qui ont une forte affinité pour le collagène (Caravan et al. 2007). Les résultats présentés à ce jour sont convaincants (Fuchs et al. 2013 ; Caravan et al. 2013), mais les questions relatives à l'administration et à la toxicité de ces agents vont très certainement restreindre leur utilisation aux modèles animaux et expliquent l'impact relativement limité de cette méthode.

Le développement de séquences à temps d'écho "ultra court " (UTE) représente, de loin, la méthode la plus prometteuse pour une imagerie directe de la fibrose. Ces séquences opèrent à des TEs très proches de zéro. Elles sont alors capables de collecter le signal RMN des spins ayant un T2 très court, jusqu'à quelques $\mu$ s (Tyler et al. 2007). Les séquences UTE, qui possèdent ce potentiel de visualiser directement la fibrose ont donc un avantage majeur sur les méthodes présentées précédemment qui sont, elles, basées sur des liens indirects avec le phénomène étudié. Les composantes à T2 court peuvent être mises en évidence par simple soustraction d'une image de référence obtenue à un TE plus long, par une saturation double-bande, un module d'inversion 


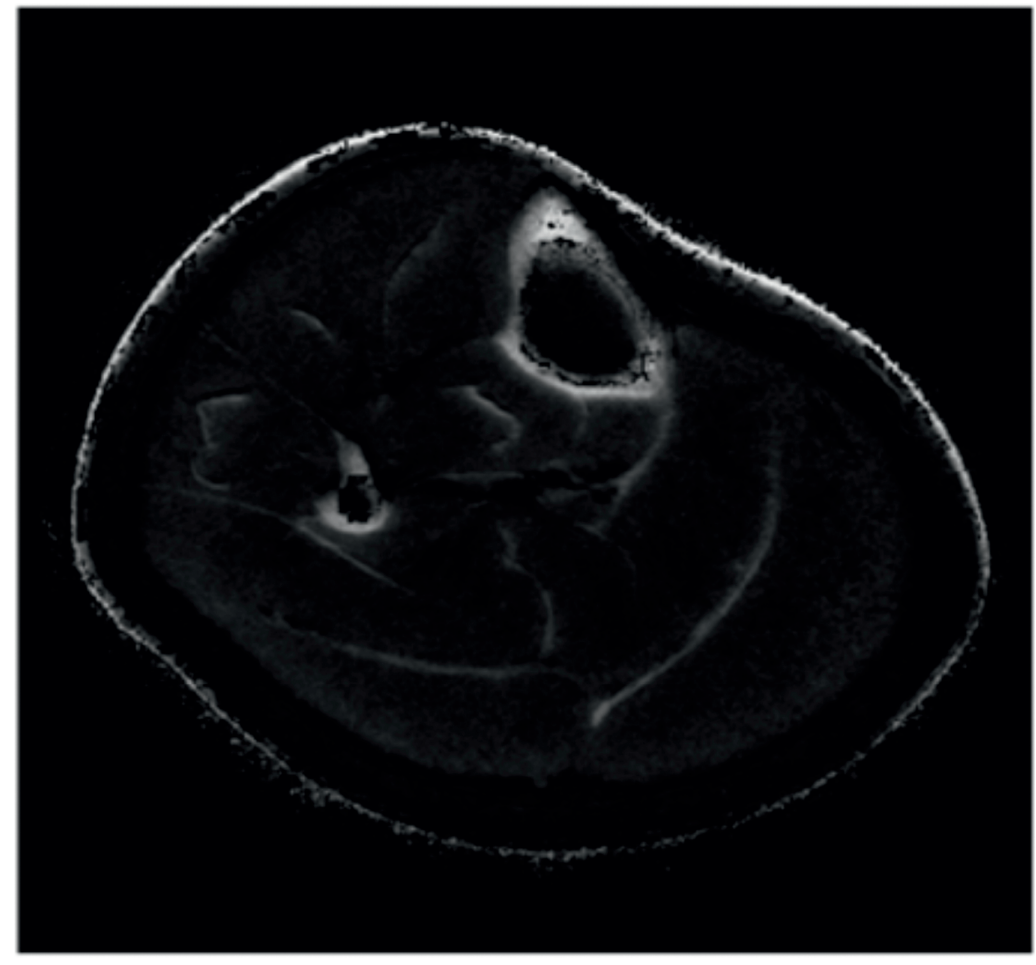

Figure 12

Exemple d'imagerie des spins à TE ultra-court (UTE), ici de la composante à T2*inférieure à une ms. Elle montre la proportion élevée de ces spins dans l'os compact, dans les fascia et dans la peau, mais aussi une fraction significative dans le tissu musculaire. La possibilité d'évaluer de manière atraumatique par cette technique UTE la composante conjonctive du muscle strié squelettique est en cours d'exploration dans plusieurs laboratoires.

adiabatique (Li et al. 2012) ou, encore mieux, par extraction de la fraction à T2 très court grâce à un ajustement multi-exponentiel du signal obtenu avec ces séquences à différents TEs, et reconstruction de cartes paramétriques qui peuvent corriger les effets de relaxation T2*, mais aussi les effets dus à la présence de lipides (Wang et al. 2010 ; Caldas de A. Araujo et al. 2015) (voir Figure 12).

L'imagerie UTE a démontré sa capacité à visualiser de manière non ambiguë l'os cortical, les couches profondes des cartilages, les tendons et les aponévroses (Robson et Bydder 2006 ; Du et al. 2009 ; $\mathrm{Du}$ et al. 2011). La visualisation directe de l'infarctus du myocarde a également été établie avec cette technique (de Jong et al. 2011). Concernant le muscle squelettique, la visualisation des tissus conjonctifs ainsi que des tentatives de quantification ont déjà été réalisées sur des sujets normaux, sur des groupes de personnes âgées, ainsi que chez des patients myopathes (Vignaud et al. 2014 ; Csapo et al. 2014 ; Caldas de A. Araujo et al. 2015). De nombreux problèmes restent tout de même à élucider, en particulier la possible superposition d'une composante lipidique à T2 ultra-court. Néanmoins, le défi le plus important sera d'isoler la fraction de molécules à T2 très court représentatifs du tissu conjonctif. La même problématique concerne également les autres approches, en particulier les expériences de transfert d'aimantation, pour lesquelles une part significative du contraste provient des protéines de l'appareil contractile. Comme mentionné précédemment, la combinaison du filtrage double quantum, de transfert d'aimantation et des séquences UTE pourrait représenter une solution pour isoler plus spécifiquement le signal provenant du collagène (Kusmia et al. 2013; Eliav et al. 2014).

\section{- Perfusion et oxygénation}

L'imagerie fonctionnelle par RMN est désormais la référence pour les explorations atraumatiques de la physiopathologie cérébrale en neurologie, aussi bien que pour les études cognitives en neuroscience (Ogawa et al. 1990 ; Detre et Wang 2002). Des approches très similaires ont été développées et appliquées avec succès au muscle squelettique (Carlier et al. 2006 ; Partovi et al. 2015). La perfusion tissulaire peut être mesurée de façon atraumatique au moyen d'un groupe de techniques qui marquent magnétiquement le sang artériel en amont du tissu d'intérêt (techniques dites "ASL"). Ce marquage résulte en une modification de l'aimantation du tissu proportionnelle à la perfusion. Cette méthode ne requiert pas d'injection d'agent de contraste, et peut donc être répétée à l'infini à un rythme très rapide, avec des intervalles de quelques secondes (Raynaud et al. 2001 ; Schewzow et al. 2014). Cette propriété est particulièrement avantageuse pour l'étude de la perfusion musculaire qui peut varier d'un facteur 20 ou plus en quelques dizaines de secondes. Le rapport contraste sur bruit en ASL est faible, et facilement contaminé par le mouvement. La plupart des études en ASL a consisté à mesurer la perfusion post-exercice ou post-ischémie. Néanmoins, si un sacrifice est réalisé sur la résolution temporelle des expériences, la perfusion au repos peut également être mesurée par ASL en moyennant le signal pendant quelques minutes (Carlier et al., 2006 ; Bertoldi et al., 2008). Le contraste BOLD (pour blood oxygen level dependant) (Ogawa et al. 1990), qui est bien connu pour sa capacité à détecter les activations cérébrales, est sensible au taux d'oxygénation des capillaires et du sang veineux dans les muscles squelettiques et peut également fournir des mesures semi-quantitatives de la saturation sanguine en oxygène (Jordan et al. 2004 ; Jacobi et al. 2012). Contrairement à la plupart des applications au niveau du cerveau, le contraste BOLD musculaire peut être négatif dans le cas d'une plus forte désaturation en oxygène, ou positif pour des situations 
où l'extraction en oxygène décroît dans les tissus (Donahue et al. 1998; Lebon et al. 1998 ; Meyer et al. 2004 ; Damon et al. 2007 ; Baligand et al. 2011). Le volume sanguin au niveau des veines et des capillaires influence le signal BOLD et rend son interprétation relativement complexe (Duteil et al. 2006). La consommation du muscle en oxygène peut être calculée, selon la loi de Fick, comme le produit de la perfusion tissulaire par la différence d'oxygénation entre artère et veine. Dans sa déclinaison RMN totalement atraumatique, la perfusion est déterminée par ASL, et le taux d'oxygénation des gros vaisseaux est obtenu par des mesures de T2 ou de susceptibilité (Zheng et al. 2013 ; Englund et al. 2013 ; Decorte et al. 2014).

Différentes conditions qui affectent la perfusion et l'oxygénation du muscle squelettique ont été étudiées par la RMN fonctionnelle, en particulier l'ischémie aiguë ou chronique (Ledermann et al. 2006 ; Grözinger et al. 2013; Aschwanden et al. 2014 ; Englund et al. 2015), mais également le diabète (Zheng et al. 2014), la sclérose systémique (Partovi et al. 2012) ou encore le syndrome des loges (Andreisek et al. 2009).

En ce qui concerne les pathologies neuromusculaires, l'emploi de l'imagerie fonctionnelle par RMN a été jusqu'à présent très limité. Chez des patients qui souffrent de glycogénose de type 3, la combinaison de l'imagerie fonctionnelle dynamique avec des mesures de spectroscopie RMN a permis de déterminer, de façon inattendue, que la diminution de la capacité de phosphorylation oxydative

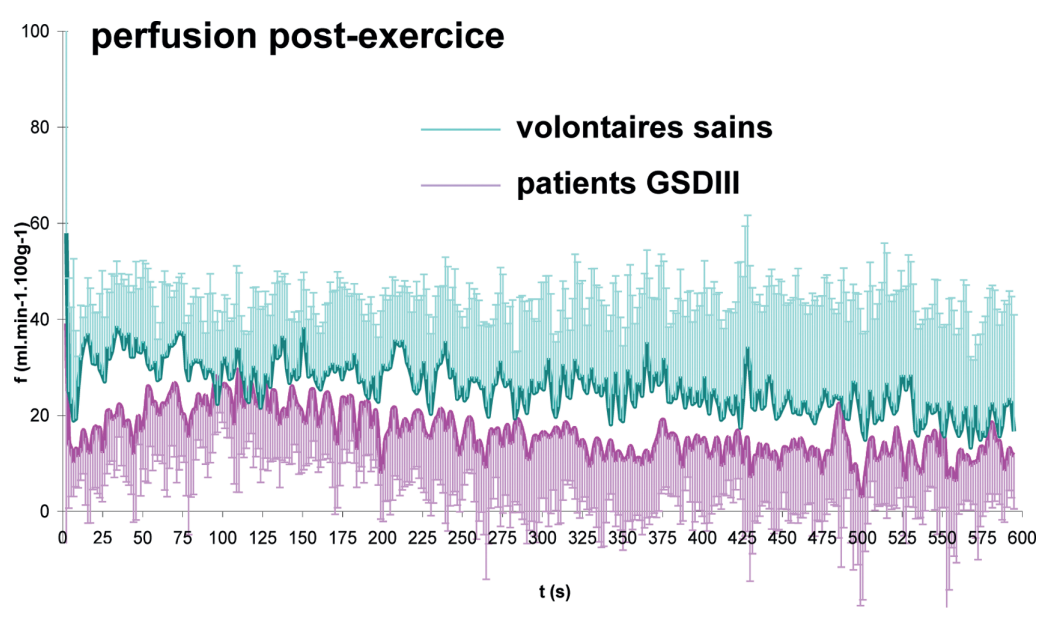

Figure 13

La mesure de la perfusion musculaire par la technique du marquage de spins de l'eau artérielle est totalement atraumatique et autorise donc de suivre très précisément la réponse hémodynamique d'un muscle au cours de différentes manœuvres. Dans l'exemple présenté ici, on voit que la perfusion musculaire post-exercice en condition aérobie est anormalement faible chez des patients adultes atteints de glycogénode de type III (déficit en enzyme débranchant). mitochondriale était au moins en partie due à une hypoperfusion musculaire relative (Wary et al. 2010) (voir Figure 13).

Il est légitime de penser que cette méthode pourra nous apporter bien plus d'informations encore dans un futur proche. Une des conséquences de la fibrose, quand celle-ci se développe aussi autour des artérioles est d'empiéter sur la réserve de vasodilatation. Dans une étude sur les muscles de la patte d'animaux hypertendus, il a été démontré que l'ASL permettait de quantifier précisément ce phénomène (Bertoldi et al. 2006). Des anomalies de la perfusion constituent également une caractéristique pathologique du muscle dystrophique chez les patients Duchenne (Thomas 2013), en partie liée à un défaut de production d'oxide nitrique (Nelson et al. 2014 ; Hörster et al. 2015), et due d'autre part aux modifications de structure liées à la fibrose (Klingler et al. 2012). La possibilité d'avoir, en recherche clinique, une mesure atraumatique et dynamique de perfusion musculaire chez des patients Duchenne représenterait un intérêt majeur, particulièrement lorsqu'il s'agira de déterminer l'efficacité de traitements à base de molécules vasodilatatrices ou anti-fibrotiques (Zhou et $\mathrm{Lu}$ 2010 ; Ennen et al. 2013 ; Blat et Blat 2015).

\section{- T1 - Pré- et post-gadolinium}

La cinétique d'évolution de la relaxivité T1 après injection d'un chélate de gadolinium à faible poids moléculaire peut être analysée de façon à obtenir une estimation quantitative de la vitesse d'échange de l'agent de contraste du compartiment vasculaire vers l'espace interstitiel (ou plus précisément vers l'espace accessible dans le compartiment extravasculaire) ainsi que du volume de ce compartiment extravasculaire (Tofts 1997; Tofts et al. 1999; Lavini et al. 2007). Idéalement, la fonction d'entrée artérielle doit être mesurée en même temps que le rehaussement du signal tissulaire. En pratique, celle-ci peut être supposée connue, ce qui facilite considérablement l'acquisition des données. L'ischémie, la nécrose, l'inflammation ou encore la fibrose peuvent être précisément évaluées à partir des modifications du taux d'échange du chélate de gadolinium et de son volume de distribution extravasculaire. Ces conditions correspondent respectivement à un échange lent et un volume de distribution normal, un échange lent et un volume augmenté, un échange rapide et un volume augmenté, et échange normal et un volume augmenté. Le muscle dystrophique canin est caractérisé par une augmentation du taux d'échange de l'agent de contraste, accompagné par une augmentation proportionnelle de son volume de distribution, ce qui se manifeste par une augmentation de l'amplitude de 
rehaussement de signal, sans décalage temporel de la prise de contraste (Thibaud et al. 2007).

Même si la procédure d'injection est peu invasive et complètement sans danger, par le recours à des agents de contraste extrêmement stables, cette technique est en perte de vitesse. Malgré la valeur ajoutée de l'information extraite par rapport à la cartographie T2 par exemple, la méthode est de nos jours rarement implémentée, à cause du coût associé, de la longueur des acquisitions nécessaires pour échantillonner convenablement les courbes de rehaussement de signal, ainsi que de la réticence à pratiquer une injection non-essentielle sur une population pédiatrique.

De la rémanence prolongée d'agents de contraste à base de gadolinium ciblant l'albumine dans le muscle dystrophique de la souris $\mathrm{mdx}$ a découlé une méthode attrayante pour caractériser la perméabilité membranaire et les dommages générés au niveau des myocytes (Straub et al. 2000 ; Amthor et al. 2004). Celle-ci reste cependant confinée aux expérimentations précliniques.

Plus récemment, un intérêt s'est manifesté pour la mesure de T1 "pré-gadolinium", probablement mieux qualifiée par T1 natif, ou intrinsèque du muscle squelettique. Ce biomarqueur a longtemps été laissé de côté car à l'époque des débuts de l'imagerie RMN du muscle strié, aucune modification du signal pondéré en T1 n'avait été relevée en présence de phénomènes inflammatoires ou nécrotiques. En réalité, les méthodes utilisées à cette époque souffraient d'un manque de sensibilité. Il a depuis été montré que le T1 natif était modérément mais significativement augmenté dans le muscle dystrophique du chien GRMD (Thibaud et al. 2012). Les cardiomyopathies dilatées et hypertrophiques engendrent également une augmentation du T1 natif, qui semble refléter les dommages cellulaires au niveau des myocytes plus que de la fibrose interstitielle (Dass et al. 2012 ; Friedrich 2015; Hinojar et al. 2015). Cette observation est corroborée par l'observation de T1 raccourcis dans des gels de collagène (Caldas de Araujo, communication personnelle). Ces pistes doivent être explorées plus en détail avant d'attribuer un rôle précis à la mesure du T1 natif pour la caractérisation du muscle squelettique. Un certain optimisme est de mise quant au futur de ce type d'approche au vu de la robustesse et la rapidité d'acquisition de séquences de quantification du T1 sur les scanners modernes.

\section{- La diffusion}

Depuis la description de son principe par Stejskal et Tanner en 1965 (Stejskal et Tanner 1965), les études de diffusion et l'imagerie de diffusion (DWI, diffusion-weighted imaging) ont été appliquées à un nombre considérable de domaines médicaux et non-médicaux, allant de l'étude des matériaux aux tissus humains, en passant par les petits animaux. La possibilité d'évaluer finement les mouvements de protons in vitro et in vivo par l'application de gradients pulsés de sensibilisation a motivé l'usage de l'imagerie de diffusion pour accéder à des paramètres structurels microscopiques, bien au-delà de la résolution de l'imagerie conventionnelle par RMN, c'est-à-dire à l'échelle du micron. En fonction du schéma d'application des gradients pulsés et de la stratégie d'analyse des données de diffusion acquises, différentes variables peuvent être calculées au sein de l'objet étudié : le coefficient de diffusion apparent et les directions de diffusion, la probabilité de déplacement ; l'échelle des barrières de diffusion (p. e. le diamètre de la cellule), et la probabilité de transition entre des compartiments délimités par des barrières semi-perméables.

Il y a environ deux décennies que l'utilisation de l'imagerie de diffusion a commencé pour l'exploration du muscle squelettique. La vaste majorité des études publiées sur le muscle squelettique étaient basées sur une séquence de diffusion EPI (echo-planar imaging) single shot. Pour l'encodage du processus de diffusion, des gradients de diffusion mono- ou bi-polaires ont été appliqués sur une séquence RMN de type spin écho. Peu d'études ont montré des résultats obtenus avec une séquence de type écho stimulé. Plusieurs difficultés doivent être surmontées pour appliquer l'imagerie de diffusion au muscle squelettique. Afin d'obtenir des variables de diffusion exactes et reproductibles, une suppression fiable et efficace du signal de la graisse est un pré-requis. La plupart des techniques disponibles (suppression de la graisse par inversion-récupération ou sélection spectrale) suppriment efficacement la composante lipidique principale, générée par les groupements méthylène. Par contre, la composante oléfinique de la graisse, très proche de la fréquence de l'eau génère des artéfacts majeurs dans l'imagerie de diffusion du muscle squelettique. De plus, l'imagerie de diffusion souffre d'un piètre rapport contraste à bruit lié à la méthode de suppression de graisse, à l'application des gradients de diffusion, et aux temps d'échos longs comparés au temps de relaxation $\mathrm{T} 2$ relativement court des protons dans le muscle squelettique. Nombre de paramètres des séquences de diffusion ont une influence majeure sur les résultats, ce qui s'est traduit par la publication dans la littérature de valeurs inconstantes pour les variables de diffusion.

Au-delà de ces particularités techniques, l'imagerie de diffusion est prometteuse et susceptible d'amener de nouveaux outils d'évaluation car plusieurs 
variables sont corrélées à des propriétés structurelles non-accessibles par d'autres techniques d'imagerie par RMN. Le coefficient de diffusion apparent, la fraction d'anisotropie, ainsi que les directions du vecteur principal de la diffusion peuvent être calculés directement à partir du tenseur de diffusion voxel par voxel. Ils ont été évalués dans plusieurs études qui se sont intéressées aux membres inférieurs et supérieurs ainsi qu'au tronc (Yanagisawa et al. 2009; Schwenzer et al. 2009b ; Froeling et al. 2012). L'analyse de l'orientation des fibres musculaires par le tenseur de diffusion s'avère corréler avec l'architecture histologique du muscle squelettique (Karampinos et al. 2009; Damon et al. 2012; Froeling etal. 2012). En utilisant des modèles de diffusion élaborés, le diamètre de la cellule musculaire ainsi que les propriétés des barrières de diffusion ont pu être calculés au départ d'images de diffusion (Sigmund et al. 2013 ; Sigmund et al. 2014). La fiabilité des variables de diffusion mesurées dans le muscle squelettique a été améliorée par l'optimisation des paramètres de séquence de diffusion ainsi que des méthodes de traitement de données diffusion (Heemskerk et al. 2010 ; Froeling etal. 2010; Froeling etal. 2013). Avec des schémas de séquence de diffusion dédiés, les variables calculées ont montré une corrélation satisfaisante avec la perfusion et la vascularisation du muscle squelettique (Karampinos et al. 2010 ; Filli et al. 2015).

La plupart des études humaines sur la diffusion musculaire ont été menées sur des volontaires sains. Peu ont exploré des pathologies musculaires (Zaraiskaya et al. 2006 ; Qi et al. 2008; Sigmund et al. 2013 ; Sigmund et al. 2014 ; Ponrartana et al. 2015) dans le contexte de l'utilisation des variables de diffusion comme biomarqueurs ou comme outils d'évaluation. Le coefficient de diffusion apparent s'est logiquement révélé anormalement élevé dans les myopathies inflammatoires, tandis que le calcul de la fraction d'anisotropie a donné des résultats divergents (Ai et al. 2014 ; Froeling et al. 2015). Il n'y a pas encore eu d'analyse longitudinale de l'activité de la maladie ni de la réponse à un traitement. Dans une étude sur la dystrophie musculaire de Duchenne, les variables de diffusion montraient une bonne corrélation avec la sévérité de la maladie et avec d'autres indicateurs RMN d'activité de la maladie (Ponrartana et al. 2015). Toutefois, aucune supériorité de la diffusion sur les variables RMN habituelles n'a été démontrée. Les altérations du coefficient de diffusion apparent et de fraction d'anisotropie dans les muscles de patients atteints de la maladie de Duchenne reflétaient avant tout leur degré d'infiltration graisseuse. Dans une autre étude récente sur la maladie de Duchenne, cette fois en prenant compte les effets de l'infiltration graisseuse et du rapport contraste à bruit, une augmentation du coefficient de diffusion apparent et de la valeur propre de diffusion lambda 3, a pu être démontrée mais dans le seul tibialis antérieur (Hooijmans et al. 2015). Dans un modèle murin de la dystrophie musculaire, les effets d'une thérapie génique ont été suivis par de nombreuses variables RMN, dont la diffusion (Park et al. 2015). La valeur ajoutée de la mesure des variables de diffusion n'a pas encore pu être déterminée. Les auteurs de cette revue pensent que l'imagerie de diffusion pourrait avoir sa place comme outil d'évaluation pour caractériser aux stades précoces de la maladie les anomalies d'orientation et d'organisation des fibres musculaires dystrophiques.

\section{- L'analyse de texture d'images}

L'analyse de texture est un domaine de recherche en soi, une niche pour spécialistes qui n'a eu jusqu'à présent que peu d'impact sur la caractérisation du tissu musculaire pathologique. La fraction graisseuse et les changements du T2 sont des biomarqueurs sensibles mais pas du tout spécifiques des maladies ou des mécanismes pathologiques sous-jacents. Il est cependant concevable que de l'information supplémentaire, sous forme de subtils motifs dans la distribution des intensités du signal de certains muscles, imperceptible à l'œil humain, soit révélée au filtre d'algorithmes d'analyse de texture.

Des mécanismes pathologiques complexes interviennent dans un muscle dystrophique, causant une désorganisation progressive des tissus. Cette perte de structure musculaire normale semble se traduire par une dispersion inhabituelle de l'intensité du signal RMN. L'hétérogénéité du signal dans un muscle dystrophique fut remarquée en premier dans le myocarde hypertrophié et défaillant d'un modèle de hamster pour la gamma-sarcoglycanopathie (Parzy et al. 2007). Des fluctuations du signal anormalement importantes mises en évidence par un coefficient de variation plus élevé pourraient être considérées comme une anomalie de texture d'ordre zéro avec une distribution spatiale aléatoire. Des observations similaires furent rapportées dans le muscle squelettique de chiens GRMD (Thibaud et al. 2012) et d'enfants Duchenne (Arpan et al. 2013 ; Wary et al. 2015a). Comme mentionné plus avant, ces observations pourraient refléter, au moins en partie, l'existence d'une fibrose musculaire, qui constitue un élément non négligeable de la désorganisation du muscle dystrophique. À un degré moindre, le vieillissement normal est associé à une augmentation faible mais significative de l'hétérogénéité du signal du muscle (Azzabou et al. 2015a), suggérant une bonne 
sensibilité de cet indicateur. Il est intéressant de noter que l'imagerie pondérée T2 combinée à des algorithmes d'analyse de texture simples permirent de discriminer des dystrophies musculaires liées à différentes mutations génétiques chez des modèles de souris (Martins-Bach et al. 2015).

Les images de muscle des patient atteints de myopathies centronucléaires héréditaires semblent présenter une texture particulière, déjà relevée à l'inspection visuelle (R.Y.Carlier, communication personnelle). Un groupe de travail a été mis en place au sein de l'action MyoMRI (www.myo-mri.eu) de la Coopération Européenne en Science et Technologie (COST), avec pour objectif précis d'identifier les motifs texturaux anormaux qui pourraient être présents dans les myopathies dystrophiques et héréditaires. Des résultats positifs ont déjà été obtenus à partir d'images de chiens GRMD (Certaines et al. 2015 ; Lerski et al. 2015). Dans cette étude, il fut montré que les matrices de co-occurrence sont des descripteurs de la texture locale plus efficaces que des indices basés sur des moments, des matrices de longueurs ou une mesure de l'anisotropie locale. Des résultats similaires furent relevés dans d'autres essais d'analyse de texture d'images RMN (Brown et al. 2015). Il faut souligner que les matrices de co-occurrence sont sensibles au nombre et à la taille des voxels acquis. Cette observation se révéla particulièrement problématique dans l'étude mentionnée ciavant. La valeur ajoutée de l'analyse de texture pour la caractérisation des anormalités du muscle squelettique au cours du temps reste à déterminer.

L'extrême dépendance des résultats de l'analyse de texture aux paramètres d'acquisition et aux particularités des plateformes demeure un obstacle à son utilisation dans des essais multi-centres. Il suffit de mentionner l'impact de la résolution spatiale sur la texture dont l'apparence sera radicalement modifiée en fonction de la taille du voxel : trop grande et la texture pourrait tout simplement disparaître, trop petite et l'augmentation du niveau de bruit pourrait la masquer. Un contrôle rigoureux et une harmonisation des paramètres d'acquisition seront un préalable indispensable à tout usage futur de l'analyse de texture comme outil d'évaluation.

\section{Les développements techniques récents et leur impact sur l'usage de la RMN comme outil d'évaluation}

L'imagerie du muscle, en particulier par RMN, est de plus en plus utilisée comme outil d'évaluation dans les essais cliniques. C'est une discipline encore relativement jeune, ayant certainement un grand potentiel, mais qui doit encore se développer, et ceci dans plusieurs directions. Il est prioritaire d'élargir l'offre d'outils disponibles pour caractériser plus précisément les muscles normaux et pathologiques. Un certain nombre d'options importantes furent présentées dans les paragraphes précédents. Les protocoles dédiés à l'étude de muscles spécifiques, en particulier dans les zones situées dans la profondeur des tissus, va aussi gagner en importance. Très peu de travaux ont jusqu'à présent été consacrés à l'évaluation de la structure et la composition in-vivo du diaphragme (Thibaud et al. 2013). La conformation anatomique ainsi que le mouvement respiratoire compliquent l'étude du diaphragme, mais son rôle fonctionnel et son implication dans de nombreuses maladies justifient ces efforts (Mead et al. 2014). Un autre exemple, parmi une multitude : la thérapie cellulaire de patients atteints de dystrophie musculaire oculo-pharyngée (Périé et al. 2014). Une imagerie de haute résolution des muscles laryngés injectés avec des myoblastes homologues pourrait permettre de suivre, sinon directement les cellules thérapeutiques éventuellement marquées avec un agent de contraste, tout au moins leur impact éventuel sur la progression des lésions dégénératives.

La quantité d'information additionnelle sur la structure et la fonction du muscle que la RMN sera capable de fournir dans le futur, et qu'elle est déjà largement capable de générer, ainsi que la multiplicité des sites anatomiques à étudier amènent une difficulté majeure : le temps nécessaire à la réalisation de ces mesures. Le problème est double : à la fois économique, au travers du coût accru de l'examen, et pratique ainsi qu'éthique, de par l'obligation pour les patients d'avoir à supporter des acquisitions de plus d'une heure. Ce dernier aspect est particulièrement pénalisant en pédiatrie. Afin de limiter le temps d'acquisition, des efforts considérables ont été consacrés, et continueront de l'être, vers 1) une accélération des acquisitions, 2) une collecte simultanée de plusieurs variables RMN et d'autant de potentiels biomarqueurs au cours d'une acquisition unique. Ces deux objectifs peuvent être poursuivis conjointement dans de nombreux cas.

\section{- Des acquisitions plus rapides}

Pour accélérer l'étape d'acquisition, l'imagerie parallèle utilise l'information spatiale capturée par des maillages d'antennes réceptrices, ce qui permet de diminuer le nombre de lignes du plan de Fourier à acquérir et ainsi de réduire d'autant le temps d'acquisition. Divers scénarios d'imagerie parallèle ont été proposés depuis presque deux décennies (Pruessmann et al. 1999; Griswold et al. 2002 ; Deshmane et al. 2012) et sont couramment sinon systématiquement mis en œuvre. Plus récemment, même si cela remonte déjà à dix ans, un nouveau 
concept a été introduit sous le nom d'acquisition comprimée (compressed sensing) (Donoho 2006 ; Lustig et al. 2008). Son principe est de tirer parti de redondances spatiales ou temporelles présentes dans certaines représentations de l'objet sousjacent, ainsi que de l'incohérence des artefacts générés par un échantillonnage irrégulier, pour éviter de remplir des pans entiers de la matrice d'acquisition tout en reconstruisant une image avec un minimum de perte de qualité. Ce principe a été récemment appliqué à l'imagerie Dixon du muscle, notamment sur des patients Becker, avec des facteurs d'accélérations pouvant atteindre 6 par comparaison aux techniques d'acquisition standard (Hollingsworth et al. 2013b; Loughran et al. 2015). La combinaison de l'acquisition comprimée avec un échantillonnage dit non-cartésien, radial par exemple, permet d'atteindre des facteurs de 10 et plus, avec pratiquement aucune perte de qualité d'image (Feng et al. 2013).

Plus spécifiquement pour la cartographie T2, de nouvelles stratégies d'acquisition ont été dérivées de séquences utilisant la précession libre en régime stationnaire (SSFP) (Bieri et al. 2011, de Sousa et al. 2012, Heule et al. 2014). Une de ces approches utilise la technique pSSFP proposée par Bieri et al. (Bieri et al. 2011). Cette méthode permet de réaliser une cartographie 3D, rapide et sélective, du T2 de l'eau du muscle squelettique, et peut être assez facilement implémentée sur un imageur commercial (de Sousa et al. 2012). Une nouvelle méthode de cartographie $\mathrm{T} 2$ rapide utilisant la technique TESS a récemment été
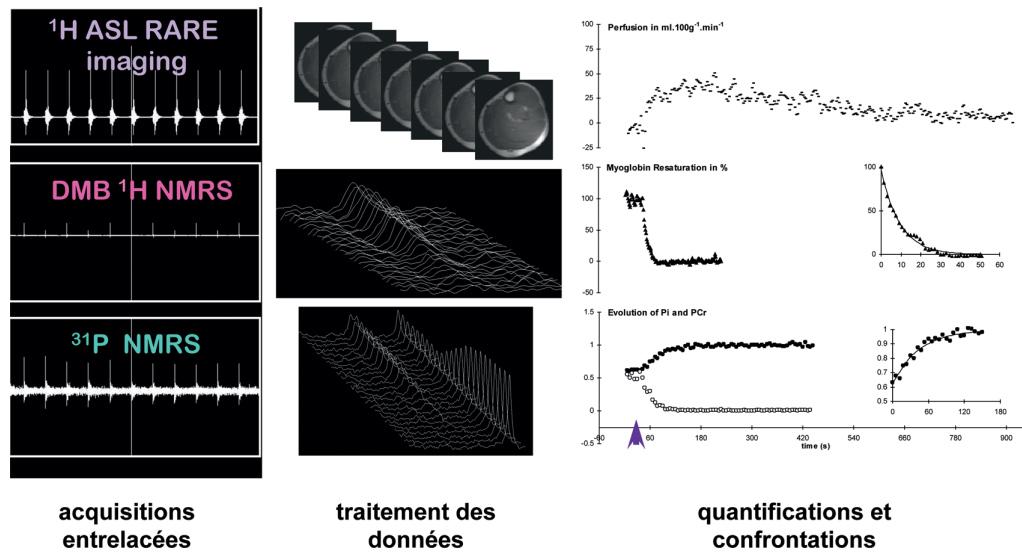

traitement des données

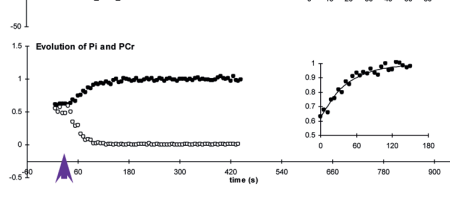

\section{quantifications et} confrontations

Figure 14

Il y a une vingtaine d'années, l'acquisition entrelacée de signaux RMN d'imagerie et de spectroscopie, provenant de plusieurs noyaux, a été mise au point sur des spectromètres de recherche et a permis des études originales du métabolisme de l'oxygène musculaire, en intégrant au cours d'un seul examen des données sur l'apport, l'extraction et la consommation d'oxygène, par la mesure simultanée de la perfusion musculaire via le marquage des spins artériels, de l'oxygénation intra-myocytaire via la spectroscopie ${ }^{1} \mathrm{H}$ de la désoxymyoglobine et de l'énergétique musculaire via la spectroscopie du ${ }^{31} \mathrm{P}$. Cette approche peut maintenant être mise en œuvre sur des imageurs cliniques, ce qui devrait élargir le champ d'applications et d'utilisations de cette technique originale. proposée pour diminuer la sensibilité au mouvement qui affecte généralement les méthodes basées sur un état stationnaire non-compensé, notamment la pSSFP (Heule et al. 2014). La TESS-T2 est nonseulement débarrassée de l'habituel biais T1/T2, commun à toutes les méthodes mono-paramétriques à état stationnaire, mais fait aussi preuve d'une grande insensibilité aux inhomogénéités de champ B1. Ces nouvelles méthodes sont bien entendu compatibles avec les techniques d'imagerie parallèle et d'acquisition comprimée.

\section{- Acquisitions simultanées de variables}

L'idée d'acquérir plusieurs variables RMN d'intérêt en une seule séquence n'est pas nouvelle (Thulborn et al. 1981 ; Eleff et al. 1988 ; Brillault-Salvat et al. 1997). C'est peut-être avec la réalisation simultanée d'une imagerie fonctionnelle de la perfusion et de l'oxygénation, ainsi que d'une spectroscopie dynamique ${ }^{1} \mathrm{H}$ et ${ }^{31} \mathrm{P}$ qu'elle a été poussée le plus loin au niveau musculaire (Duteil et al. 2004) (voir Figure 14). Une telle approche fait gagner du temps, elle autorise une analyse combinée de variables dynamiques qui n'aurait pas été possible si celles-ci avaient été acquises séparément, ceci du fait de la difficulté pour un sujet à reproduire plusieurs fois le même exercice musculaire. Il a été démontré que l'extraction d'oxygène musculaire pouvait être déduite de tels protocoles fonctionnels d'imagerie et de spectroscopie multi-paramétriques (Carlier et al. 2005). C'est à l'aide d'outils multi-paramétriques qu'il a été identifié qu'une perfusion anormale contribuait à une réduction de la capacité des oxydations phosphorylantes mitochondriales chez les patients atteints de glycogénose de type III (Wary et al. 2010).

Dans le domaine de la caractérisation tissulaire musculaire, l'acquisition simultanée de cartographies pour plusieurs variables est une direction prometteuse, toujours dans l'objectif d'accélérer les évaluations quantitatives. Notre équipe a étudié la faisabilité d'une imagerie multi-paramétrique rapide, avec des temps d'acquisition de 2 secondes par coupe. Elle a permis de suivre les adaptations rapides du muscle squelettique à divers stimuli physiologiques et métaboliques (de Sousa et al. 2011). Cette séquence est constituée d'une impulsion d'inversion suivie de l'acquisition de plusieurs images en régime SSFF équilibré pendant que le signal s'approche d'un état stationnaire (Schmitt et al. 2004). Les valeurs de T1, T2 et M0 (la densité de protons relative) peuvent alors être déduites d'une régression mono-exponentielle à trois paramètres sur la série d'images. La trajectoire du signal en IR-bSSFP peut être modélisée plus précisément à partir des équations de Bloch ou du formalisme EPG afin de 
prendre en compte certaines erreurs d'instrumentation (les inhomogénéités de B1+ et B0, l'efficacité d'inversion et les profils de coupes non-idéaux) (Hennig et al. 2004 ; Weigel 2014 ; Marty et al. 2015) et la composition des tissus (les composantes eau/graisse).

Les cartographies de fraction graisseuse et de T2 sont les deux biomarqueurs RMN les plus matures et s'affirment comme les plus utiles pour les études musculaires. Parvenir à extraire d'une même séquence l'information nécessaire à la création de cartographies de fraction graisseuse et de T2 représentera un gain de temps considérable.

Une méthode appelée IDEAL-CPMG, combinant l'algorithme IDEAL avec une séquence de Carr-Purcell-Meiboom-Gill a récemment été proposée (Janiczek et al. 2011). Les images produites sont séparées en eau et graisse pour chaque écho. On procède ensuite à une régression exponentielle sur chaque décroissance pour quantifier le T2 de l'eau et celui de la graisse. La fraction graisseuse est calculée à partir des images en densité de proton de l'eau et de la graisse obtenues via la méthode de reconstruction IDEAL. Néanmoins, des formes d'impulsion RF imparfaites et un champ RF inhomogène peuvent donner lieu à la génération d'échos stimulés et rendre l'estimation du T2 très incertaine avec des modèles purement exponentiels. De plus, la conception de cette séquence d'impulsions

- images multi-TE spin-echo (MSME) de départ

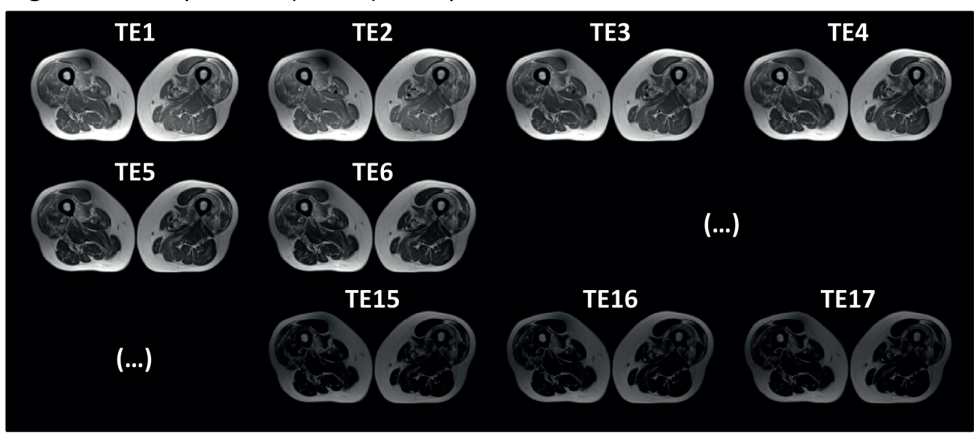

- images et cartographies construites à partir des images MSME

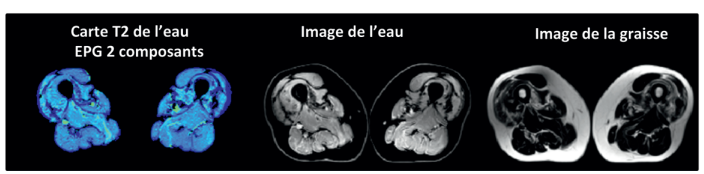

Figure 15

Les deux principaux biomarqueurs RMN musculaires, le pourcentage de graisse et le T2 de

l'eau musculaire peuvent être calculés à partir d'une seule série d'images en écho de spin qui couvre une gamme de TEs. Dans l'extraction des données, il est possible de prendre en compte les inhomogénéités de champ radio-fréquence. Cette approche, basée exclusivement sur une optimisation du post-processing, permet de réduire très significativement la durée d'un examen puisqu'elle fait l'économie d'une imagerie eau-graisse complémentaire, tout en ne demandant que la mise en ouvre d'une séquence standard, disponible sur tous les imageurs. impose l'utilisation d'une large bande passante de lecture, ce qui abaisse le rapport signal sur bruit des images produites.

Une autre approche pour mesurer simultanément le T2 de l'eau et la fraction graisseuse est de procéder à une régression multi-composante sur la séquence standard MSME en tirant partie des différences de relaxations T1 et T2 entre les protons de l'eau et de la graisse. Des modèles exponentiels peuvent être utilisés pour décrire la décroissance du signal MSME, comme dans les travaux d'Azzabou et al. (Azzabou et al. 2015). Dernièrement, notre équipe a proposé d'utiliser uniquement la séquence MSME et une régression de modèles à base d'EPG pour extraire simultanément la fraction de graisse et le T2 de l'eau (Marty et al. 2016). La validation de cette approche par rapport à des acquisitions séparées est en cours et, espérons-le, justifiera son implémentation dans des protocoles en permettant une réduction du temps d'acquisition d'environ $50 \%$ (voir Figure 15).

\section{Conclusions}

En dépit de coûts élevés, de l'impossibilité d'intervenir au lit du patient, d'une technologie complexe élevée et d'une interprétation parfois ardue, le rôle de l'imagerie et la spectroscopie par RMN comme outil d'évaluation s'affirme progressivement dans les études musculaires. S'il paraît accuser un retard par rapport à la contribution de l'imagerie dans l'évaluation d'autres organes, tels que le cerveau, c'est le résultat d'une plus faible incidence des pathologies musculaires, d'un accès limité aux imageurs et du manque de perspectives thérapeutiques, ces deux derniers obstacles sont progressivement en train d'être levés. Les cartographies paramétriques de la fraction graisseuse et du T2 fournissent des mesures atraumatiques et quantitatives, respectivement des lésions dégénératives du muscle squelettique et de l'activité de la maladie. Ils sont de loin les deux biomarqueurs RMN les plus utilisés dans les protocoles musculaires. En dépit des limitations et contraintes mentionnées plus haut, la valeur ajoutée d'une telle caractérisation a conduit progressivement à l'intégration quasi systématique de l'imagerie RMN comme outil d'évaluation dans les essais cliniques récents. Les développements actuels visent à raccourcir les séquences d'acquisitions et à collecter en même temps fraction graisseuse et T2, et vont considérablement diminuer le temps d'examen. Leur implémentation dans des protocoles multicentriques est attendue dans les prochains mois et années, et va grandement améliorer la compliance des patients, notamment pédiatriques, à ce type d'examen. Accélérer les acquisitions des cartographies de fraction 
graisseuse et de T2 pourra aussi dégager du temps pour inclure la mesure d'autres biomarqueurs dans des protocoles cliniques, comme par exemple le contenu en phospho-diesters par spectroscopie du ${ }^{31} \mathrm{P}$, la perfusion musculaire par marquage de spins artériels et/ou la fibrose interstitielle avec des séquences UTE. Explorer les muscles squelettiques, superficiels ou profonds, et caractériser l'anatomie, la structure, la biochimie et la fonction du muscle de façon atraumatique, et potentiellement simultanément, sont spécifiques à la RMN. Grâce aux innovations technologiques sans cesse introduites, ces propriétés devraient, dans un futur proche, être de plus en plus exploitées dans des contextes cliniques et viendront renforcer le rôle de la RMN comme outil d'évaluation dans les essais thérapeutiques sur le muscle squelettique.

\section{Abstract}

Recent years have seen tremendous progress towards therapy of many previously incurable neuromuscular diseases. This new context has acted as a driving force for the development of novel non-invasive outcome measures. These can be organized in three main categories: functional tools, fluid biomarkers and imagery. In the latest category, nuclear magnetic resonance imaging (NMRI) offers a considerable range of possibilities for the characterization of skeletal muscle composition, function and metabolism. Nowadays, three NMR outcome measures are frequently integrated in clinical research protocols. They are: $1 /$ the muscle cross sectional area or volume, 2/ the percentage of intramuscular fat and 3/ the water T2 maps, which quantity muscle trophicity, chronic fatty degenerative changes and edema (or more broadly, "disease activity"), respectively. A fourth biomarker, the contractile tissue volume is easily derived from the first two ones. The fat fraction maps most often acquired with Dixon sequences have proven their capability to detect small changes in muscle composition and have repeatedly shown superior sensitivity over standard functional evaluation. This outcome measure will more than likely be the first of the series to be validated as an endpoint by regulatory agencies. The versatility of contrast generated by NMR has opened many additional possibilities for characterization of the skeletal muscle and will result in the proposal of more NMR biomarkers. Ultra-short TE (UTE) sequences, late gadolinium enhancement and NMR elastography are being investigated as candidates to evaluate skeletal muscle interstitial fibrosis. Many options exist to measure muscle perfusion and oxygenation by NMR. Texture analysis algorithms could generate complementary information on muscle organization at a mesoscopic scale. ${ }^{31} \mathrm{P}$ NMR spectroscopy is the reference technique to asses muscle energetics non-invasively during and after exercise. In dystrophic muscle, ${ }^{31} \mathrm{P}$ NMR spectrum at rest is profoundly perturbed, and several resonances inform on cell membrane integrity. Considerable efforts are being directed towards acceleration of image acquisitions using a variety of approaches, from the extraction of fat content and water T2 maps from one single acquisition to partial matrices acquisition schemes. Spectacular decreases in examination time are expected in the near future. They will reinforce the attractiveness of imaging outcome measures and will further facilitate their integration in clinical research trials.

Key words : magnetic resonance imaging - MRI magnetic resonance spectroscopy - outcome measure clinical trials

Skeletal muscle quantitative nuclear magnetic resonance imaging and spectroscopy as an outcome measure for clinical trials

\section{LIENS D'INTÉR̂̂T}

Pierre Carlier a participé à des réunions du conseil scientifique de Genzyme-Sanofi et Biomarin-Prosensa, pour lesquelles il a perçu des honoraires. Il est intervenu ou intervient bénévolement comme conseiller scientifique de Servier, Atyr, Tarix, Esperare, Roche et CRIS

Olivier Scheidegger a bénéficié d'aides de Genzyme et de Bayer pour la participation à des congrès.

Les autres auteurs déclarent n'avoir aucun lien d'intérêt concernant les données publiées dans cet article.

\section{RÉFÉRENCES}

Ai T, Yu K, Gao L, et al. Diffusion tensor imaging in evaluation of thigh muscles in patients with polymyositis and dermatomyositis. Br J Radiol 2014 ; 87 (1043) : 20140261.

Alexander, Matthew S, Kunkel, et al. Skeletal Muscle MicroRNAs: Their diagnostic and therapeutic potential in human muscle disease. J Neuromuscul Dis 2015 ; 2 : 1-11.

Alizai H, Nardo L, Karampinos DC, et al. Comparison of clinical semi-quantitative assessment of muscle fat infiltration with quantitative assessment using chemical shift-based water/fat separation in MR studies of the calf of post-menopausal women. Eur Radiol $2012 ; 22: 1592-600$

Amthor H, Egelhof T, McKinnell I, et al. Albumin targeting of damaged muscle fibres in the mdx mouse can be monitored by MRI. Neuromuscul Disord 2004 ; 14 : 791-6.

Andreisek G, White LM, Sussman MS, et al. T2*-weighted and arterial spin labeling MRI of calf muscles in healthy volunteers and patients with chronic exertional compartment syndrome: preliminary experience. Am J Roentgenol 2009; 193 : 327-33.

Araujo ECA, Fromes Y, Carlier PG. New Insights on Human Skeletal Muscle Tissue Compartments Revealed by In Vivo T2 NMR Relaxometry. Biophys J 2014 ; 106 : 2267-74.

Arechavala-Gomeza V, Anthony K, Morgan J, Muntoni F. Antisense oligonucleotide-mediated exon skipping for Duchenne muscular dystrophy: progress and challenges. Curr Gene Ther 2012 ; $12: 152-60$

Argov Z, Löfberg M, Arnold DL. Insights into muscle diseases gained by phosphorus magnetic resonance spectroscopy. Muscle Nerve $2000 ; 23$ : 1316-34.

Arpan I, Forbes SC, Lott DJ, et al. T2 mapping provides multiple approaches for the characterization of muscle involvement in neuromuscular diseases: a cross-sectional study of lower leg muscles 
in 5-15-year-old boys with Duchenne muscular dystrophy. NMR Biomed 2013; 26:320-8.

Arpan I, Willcocks RJ, Forbes SC, et al. Examination of effects of corticosteroids on skeletal muscles of boys with DMD using MRI and MRS. Neurology 2014; 83 : 974-80

Aschwanden M, Partovi S, Jacobi B, et al. Assessing the endorgan in peripheral arterial occlusive disease-from contrastenhanced ultrasound to blood-oxygen-level-dependent MR imaging. Cardiovasc Diagn Ther 2014 ; 4 : 165-72.

Azzabou N, Carlier PG Fat quantification and T2 measurement. Pediatr Radiol 2014 ; 44 : 1620-1.

Azzabou N, Hogrel J-Y, Carlier PG. NMR based biomarkers to study age-related changes in the human quadriceps. Exp Gerontol 2015a ; 70 : 54-60.

Azzabou N, Loureiro de Sousa P, Caldas E, Carlier PG. Validation of a generic approach to muscle water T2 determination at 3T in fat-infiltrated skeletal muscle. J Magn Reson Imaging 2015b ; 41 : 645-53.

Azzabou N, Sousa PL de, Caldas E, Carlier PG. Validation of a generic approach to muscle water T2 determination at 3T in fatinfiltrated skeletal muscle. $J$ Magn Reson Imaging 2015c ; 41 : 645-53.

Baligand C, Wary C, Ménard JC, et al. Measuring perfusion and bioenergetics simultaneously in mouse skeletal muscle: a multiparametric functional-NMR approach. NMR Biomed 2011; 24 281-90.

Barbiroli B, Funicello R, Ferlini A, et al. Muscle energy metabolism in female DMD/BMD carriers: a 31P-MR spectroscopy study. Muscle Nerve 1992a ; 15 : 344-48.

Barbiroli B, Funicello R, Iotti S, et al. 31P-NMR spectroscopy of skeletal muscle in Becker dystrophy and DMD/BMD carriers. Altered rate of phosphate transport. J Neurol Sci 1992b ; 109 188-95.

Barbiroli B, McCully KK, Iotti S, et al. Further impairment of muscle phosphate kinetics by lengthening exercise in DMD/BMD carriers. An in vivo 31P-NMR spectroscopy study. $J$ Neurol Sci $1993 ; 119: 65-73$.

Barnouin Y, Butler-Browne G, Voit T, et al. Manual segmentation of individual muscles of the quadriceps femoris using MRI: a reappraisal. J Magn Reson Imaging 2014 ; 40 : 239-47.

Bertoldi D, Parzy E, Fromes Y, et al. New insight into abnormal muscle vasodilatory responses in aged hypertensive rats by in vivo nuclear magnetic resonance imaging of perfusion. $J$ Vasc Res 2006 ; 43 : 149-56.

Bertoldi D, Loureiro de Sousa P, Fromes Y, et al. Quantitative, dynamic and non-invasive determination of skeletal muscle perfusion in mouse leg by NMR arterial spin-labeled imaging. Magn Reson Imaging 2008 ; 26 : 1259-65.

Bieri O, Scheffler K, Welsch GH, et al. Quantitative mapping of T2 using partial spoiling. Magn Reson Med 2011 ; 66 : 410-8.

Blat Y, Blat S. Drug Discovery of Therapies for Duchenne Muscular Dystrophy. J Biomol Screen 2015; 10 : 1189-203. Epub 2015 May 14

Bley TA, Wieben O, François CJ, et al. Fat and water magnetic resonance imaging. J Magn Reson Imaging 2010 ; 31 : 4-18.

Boesch C. Musculoskeletal spectroscopy. J Magn Reson imaging 2007 ; 25 : 321-38

Bonati U, Hafner P, Schädelin S, et al. Quantitative muscle MRI A powerful surrogate outcome measure in Duchenne muscular dystrophy. Neuromuscul Disord 2015a ; 25 : 679-85.

Bonati U, Schmid M, Hafner P, et al. Longitudinal 2-point dixon muscle magnetic resonance imaging in becker muscular dystrophy. Muscle Nerve 2015b ; 51 : 918-21.

Brillault-Salvat C, Giacomini E, Jouvensal L, et al. Simultaneous determination of muscle perfusion and oxygenation by interleaved NMR plethysmography and deoxymyoglobin spectroscopy. NMR Biomed 1997 ; 10 : 315-23

Brown AM, Nagala S, McLean MA, et al. Multi-institutional validation of a novel textural analysis tool for preoperative stratification of suspected thyroid tumors on diffusion-weighted MRI. Magn Reson Med 2015; doi: 10.1002/mrm.25743

Bryan WW, Reisch JS, McDonald G, et al. Magnetic resonance imaging of muscle in amyotrophic lateral sclerosis. Neurology $1998 ; 51: 110-3$
Bryant ND, Li K, Does MD, et al. Multi-parametric MRI characterization of inflammation in murine skeletal muscle. NMR Biomed $2014 ; 27$ : 716-25.

Bushby K, Finkel R, Wong B, et al. Ataluren treatment of patients with nonsense mutation dystrophinopathy. Muscle Nerve 2014 ; $50: 477-87$

Buyse GM, Voit T, Schara U, et al. Efficacy of idebenone on respiratory function in patients with Duchenne muscular dystrophy not using glucocorticoids (DELOS) : a double-blind randomised placebo-controlled phase 3 trial. Lancet 2015 ; 385 : 1748-57.

Caldas de A. Araujo E, Azzabou N, Vignaud A, et al. Quantitative NMR imaging of the short-T2 components in the SKM tissue: alterations observed in myopathic patients. In: ISMRM 23rd Annual Meeting \& Exhibition. Toronto, Ontario, Canada, 2015; p. 251. Caravan P, Das B, Dumas S, et al. Collagen-targeted MRI contrast agent for molecular imaging of fibrosis. Angew Chem Int Ed Engl $2007 ; 46: 8171-3$

Caravan P, Yang Y, Zachariah R, et al. Molecular magnetic resonance imaging of pulmonary fibrosis in mice. Am $J$ Respir Cell Mol Biol 2013 ; 49 : 1120-6.

Carlier PG. Global T2 versus water T2 in NMR imaging of fatty infiltrated muscles: different methodology, different information and different implications. Neuromuscul Disord 2014; 24 : 390-2.

Carlier PG, Azzabou N, de Sousa PL, et al. P.14.4 Diagnostic role of quantitative NMR imaging exemplified by 3 cases of juvenile dermatomyositis. Neuromuscul Disord 2013; $23: 814$.

Carlier PG, Azzabou N, de Sousa PL, et al. Skeletal muscle quantitative nuclear magnetic resonance imaging follow-up of adult Pompe patients. J Inherit Metab Dis 2015 ; 38 : 565-72.

Carlier PG, Bertoldi D, Baligand C, et al. Muscle blood flow and oxygenation measured by NMR. NMR Biomed 2006; 19 : 954-67.

Carlier PG, Brillault-Salvat C, Giacomini E, et al. How to investigate oxygen supply, uptake, and utilization simultaneously by interleaved NMR imaging and spectroscopy of the skeletal muscle. Magn Reson Med 2005 ; 54 : 1010-3.

Carlier PG, Shukelovich A, Baudin PY, et al. Fast, precise, interactive segmentation of skeletal muscle NMR images. Neuromuscul Disord 2014 ; 24 : 836-37.

Certaines JD De, Larcher T, Duda D, et al. Application of texture analysis to muscle MRI: 1-What kind of information should be expected from texture analysis? EPJ Nonlinear Biomed Phys $2015 ; 1-3: 1-14$

Chance B, Younkin DP, Kelley R, et al. Magnetic resonance spectroscopy of normal and diseased muscles. Am J Med Genet 1986 ; $25: 659-79$.

Cirak S, Arechavala-Gomeza V, Guglieri M, et al. Exon skipping and dystrophin restoration in patients with Duchenne muscular dystrophy after systemic phosphorodiamidate morpholino oligomer treatment: an open-label, phase 2, dose-escalation study. Lancet $2011 ; 378$ : 595-605.

Csapo R, Malis V, Sinha U, et al. Age-associated differences in triceps surae muscle composition and strength - an MRI-based cross-sectional comparison of contractile, adipose and connective tissue. BMC Musculoskelet Disord 2014 ; 15 : 209 Epub 2014 Jun 17.

Damon BM, Heemskerk AM, Ding Z. Polynomial fitting of DT-MRI fiber tracts allows accurate estimation of muscle architectural parameters. Magn Reson Imaging 2012 ; 30 : 589-600.

Damon BM, Wadington MC, Hornberger JL, Lansdown DA. Absolute and relative contributions of BOLD effects to the muscle functional MRI signal intensity time course: effect of exercise intensity. Magn Reson Med 2007 ; 58 : 335-45.

Dass S, Suttie JJ, Piechnik SK, et al. Myocardial Tissue Characterization Using Magnetic Resonance Noncontrast T1 Mapping in Hypertrophic and Dilated Cardiomyopathy. Circ Cardiovasc Imaging $2012 ; 5$ : 726-33

de Jong S, Zwanenburg JJ, Visser F, et al. Direct detection of myocardial fibrosis by MRI. $J$ Mol Cell Cardiol 2011; 51 : 974-79.

de Sousa PL, Vignaud A, Caldas de Almeida Araújo E, Carlier PG. Factors controlling T2 mapping from partially spoiled SSFP 
sequence: optimization for skeletal muscle characterization. Magn Reson Med 2012 ; 67 : 1379-90

de Sousa PL, Vignaud A, Fleury S, Carlier PG. Fast monitoring of $\mathrm{T}(1), \mathrm{T}(2)$, and relative proton density $(\mathrm{M}(0))$ changes in skeletal muscles using an IR-TrueFISP sequence. $J$ Magn Reson imaging 2011; 33 : 921-30.

Decorte N, Buehler T, Caldas de Almeida Araujo E, et al. Noninvasive estimation of oxygen consumption in human calf muscle through combined NMR measurements of ASL perfusion and $\mathrm{T}_{2}$ oxymetry. J Vasc Res $2014 ; 51: 360-8$.

Decostre V, Canal A, Ollivier G, et al. Wrist flexion and extension torques measured by highly sensitive dynamometer in healthy subjects from 5 to 80 years. BMC Musculoskelet Disord 2015; 16 : 4. Epub 2015 Jan 31.

Degardin A, Morillon D, Lacour A, et al. Morphologic imaging in muscular dystrophies and inflammatory myopathies. Skeletal Radiol 2010 ; 39 : 1219-27.

Desguerre I, Mayer M, Leturcq F, et al. Endomysial Fibrosis in Duchenne Muscular Dystrophy. J Neuropathol Exp Neurol 2009; 68 : 762-73.

Deshmane A, Gulani V, Griswold MA, Seiberlich N. Parallel MR imaging. J Magn Reson Imaging 2012 ; 36 : 55-72.

Detre JA, Wang J. Technical aspects and utility of fMRI using BOLD and ASL. Clin Neurophysiol 2002; 113: 621-34.

Donahue KM, Van Kylen J, Guven S, et al. Simultaneous gradientecho/spin-echo EPI of graded ischemia in human skeletal muscle. $J$ Magn Reson Imaging 1998 ; 8 : 1106-13.

Donoho DL. Compressed sensing. IEEE Trans Inf Theory 2006 ; 1289-306.

Dowling P, Holland, Ashling, Ohlendieck K. Mass spectrometrybased identification of muscle-associated and muscle-derived proteomic biomarkers of dystrophinopathies. $J$ Neuromuscul Dis $2014 ; 1: 15-40$.

Douglas AGL, Wood MJA. Splicing therapy for neuromuscular disease. Mol Cell Neurosci 2013; 56 : 169-85.

Drakonaki EE, Allen GM, Wilson DJ. Ultrasound elastography for musculoskeletal applications. Br J Radiol 2012 ; 85 : 1435-45.

Du J, Bydder M, Takahashi AM, et al. Short T2 contrast with three-dimensional ultrashort echo time imaging. Magn Reson Imaging $2011 ; 29: 470-82$.

Du J, Takahashi AM, Chung CB. Ultrashort TE spectroscopic imaging (UTESI): application to the imaging of short T2 relaxation tissues in the musculoskeletal system. $J$ Magn Reson Imaging 2009; $29: 412-21$.

Duteil S, Bourrilhon C, Raynaud JS, et al. Metabolic and vascular support for the role of myoglobin in humans: a multiparametric NMR study. Am J Physiol Regul Integr Comp Physiol 2004 ; 287: R1441-9.

Duteil S, Wary C, Raynaud JS, et al. Influence of vascular filling and perfusion on BOLD contrast during reactive hyperemia in human skeletal muscle. Magn Reson Med 2006 ; 55 : 450-4.

e Lima KMM, da Matta TT, de Oliveira LF. Reliability of the rectus femoris muscle cross-sectional area measurements by ultrasonography. Clin Physiol Funct Imaging 2012 ; 32 : 221-6.

Edwards RH, Dawson MJ, Wilkie DR, et al. Clinical use of nuclear magnetic resonance in the investigation of myopathy. Lancet $1982 ; 725-30$.

Edzes H, Samulski ET. The measurement of cross-relaxation effects in the proton NMR spin-lattice relaxation of water in biological systems: Hydrated collagen and muscle. J Magn Reson 1978; $31: 207-29$.

Eleff SM, Schnall MD, Ligetti L, et al. Concurrent measurements of cerebral blood flow, sodium, lactate, and high-energy phosphate metabolism using 19F, 23Na, 1H, and 31P nuclear magnetic resonance spectroscopy. Magn Reson Med 1988 ; 7 : 412-24.

Eliav U, Komlosh ME, Basser PJ, Navon G. Collagen composition and content-dependent contrast in porcine annulus fibrosus achieved by using double quantum and magnetization transfer filtered UTE MRI. Magn Reson Med 2014 ; 71 : 388-93.

Englund EK, Langham MC, Li C, et al. Combined measurement of perfusion, venous oxygen saturation, and skeletal muscle $\mathrm{T} 2 *$ during reactive hyperemia in the leg. $J$ Cardiovasc Magn Reson $2013 ; 15: 70$.
Englund EK, Langham MC, Ratcliffe SJ, et al. Multiparametric assessment of vascular function in peripheral artery disease: dynamic measurement of skeletal muscle perfusion, blood-oxygenlevel dependent signal, and venous oxygen saturation. Circ Cardiovasc Imaging 2015; doi: 10.1161/CIRCIMAGING.114. 002673.

Ennen JP, Verma M, Asakura A. Vascular-targeted therapies for Duchenne muscular dystrophy. Skelet Muscle 2013 ; 3-9: 1-12. Erriquez D, Perini G, Ferlini A. Non-coding RNAs in muscle dystrophies. Int J Mol Sci 2013; 14 : 19681-704.

Feng L, Grimm R, Block KT, et al. Golden-angle radial sparse parallel MRI: Combination of compressed sensing, parallel imaging, and golden-angle radial sampling for fast and flexible dynamic volumetric MRI. Magn Reson Med 2013 ; 72 : 707-17.

Filli L, Boss A, Wurnig MC, et al. Dynamic intravoxel incoherent motion imaging of skeletal muscle at rest and after exercise. NMR Biomed 2015; 28 : 240-6.

Fischmann A, Hafner P, Fasler S, et al. Quantitative MRI can detect subclinical disease progression in muscular dystrophy. $J$ Neurol 2012; 259 : 1648-54.

Fischmann A, Hafner P, Gloor M, et al. Quantitative MRI and loss of free ambulation in Duchenne muscular dystrophy. $J$ Neurol $2013 ; 260: 969-74$

Fischmann A, Morrow JM, Sinclair CDJ, et al. Improved anatomical reproducibility in quantitative lower-limb muscle MRI. J Magn Reson imaging 2014 ; 39 : 1033-8.

Fleckenstein JL, Watumull D, Conner KE, et al. Denervated human skeletal muscle: MR imaging evaluation. Radiology 1993 ; $187: 213-8$

Forbes SC, Willcocks RJ, Triplett WT, et al. Magnetic resonance imaging and spectroscopy assessment of lower extremity skeletal muscles in boys with Duchenne muscular dystrophy: a multicenter cross sectional study. PLoS One 2014 ; 9 : e106435.

Friedrich MG. Myocardial T1: The Rise of a Novel Biomarker Continues. JACC Cardiovasc Imaging 2015 ; 8 : 47-49.

Froeling M, Nederveen AJ, Heijtel DFR, et al. Diffusion-tensor MRI reveals the complex muscle architecture of the human forearm. J Magn Reson Imaging 2012 ; 36 : 237-48.

Froeling M, Nederveen AJ, Nicolay K, Strijkers GJ. DTI of human skeletal muscle: the effects of diffusion encoding parameters, signal-to-noise ratio and T2 on tensor indices and fiber tracts. NMR Biomed 2013; $26: 1339-52$.

Froeling M, Oudeman J, Strijkers GJ, et al. Muscle changes detected with diffusion-tensor imaging after long-distance running. Radiology 2015 ; 274 : 548-62.

Froeling M, Oudeman J, van den Berg S, et al. Reproducibility of diffusion tensor imaging in human forearm muscles at $3.0 \mathrm{~T}$ in a clinical setting. Magn Reson Med 2010 ; 64 : 1182-90.

Fuchs BC, Wang H, Yang Y, et al. Molecular MRI of collagen to diagnose and stage liver fibrosis. J Hepatol 2013 ; 59 : 992-8.

Gaeta M, Messina S, Mileto A, et al. Muscle fat-fraction and mapping in Duchenne muscular dystrophy: evaluation of disease distribution and correlation with clinical assessments. Preliminary experience. Skeletal Radiol 2012; 41 : 955-61.

Garrood P, Hollingsworth KG, Eagle M, et al. MR imaging in Duchenne muscular dystrophy: quantification of T1-weighted signal, contrast uptake, and the effects of exercise. J Magn Reson imaging $2009 ; 30: 1130-8$.

Gineste C, De Winter JM, Kohl C, et al. In vivo and in vitro investigations of heterozygous nebulin knock-out mice disclose a mild skeletal muscle phenotype. Neuromuscul Disord 2013a; 23 : 357-69.

Gineste C, Duhamel G, Le Fur Y, et al. Multimodal MRI and (31)P-MRS investigations of the ACTA1(Asp286Gly) mouse model of nemaline myopathy provide evidence of impaired in vivo muscle function, altered muscle structure and disturbed energy metabolism. PLoS One 2013b; 8 : e72294.

Gineste C, Le Fur Y, Vilmen C, et al. Combined MRI and 31P-MRS investigations of the ACTA1(H40Y) mouse model of nemaline myopathy show impaired muscle function and altered energy metabolism. PLoS One 2013c ; 8 : e61517.

Glaser KJ, Manduca A, Ehman RL. Review of MR elastography applications and recent developments. J Magn Reson imaging $2012 ; 36$ : 757-74. 
Griswold MA, Jakob PM, Heidemann RM, et al. Generalized autocalibrating partially parallel acquisitions (GRAPPA). Magn Reson Med 2002; $47: 1202-10$.

Grözinger G, Pohmann R, Schick F, et al. Perfusion measurements of the calf in patients with peripheral arterial occlusive disease before and after percutaneous transluminal angioplasty using MR arterial spin labeling. J Magn Reson imaging 2013 ; $40: 980-7$.

Ha D-H, Choi S, Kang E-J, Park HT. Diffusion tensor imaging and T2 mapping in early denervated skeletal muscle in rats. $J$ Magn Reson Imaging $2015 ; 42: 617-23$

Heemskerk AM, Sinha TK, Wilson KJ, et al. Repeatability of DTIbased skeletal muscle fiber tracking. NMR Biomed 2010; 23 : 294-303.

Heemskerk AM, Strijkers GJ, Drost MR, et al. Skeletal muscle degeneration and regeneration after femoral artery ligation in mice monitoring with diffusion MR imaging. Radiology 2007; 243 413-21.

Heerschap A, Houtman C, in 't Zandt HJ, et al. Introduction to in vivo 31P magnetic resonance spectroscopy of (human) skeletal muscle. Proc Nutr Soc 1999; 58 : 861-70.

Heier CR, Guerron AD, Korotcov A, et al. Non-invasive MRI and spectroscopy of mdx mice reveal temporal changes in dystrophic muscle imaging and in energy deficits. PLoS One 2014; 9 : e112477.

Henkelman RM, Stanisz GJ, Graham SJ. Magnetization transfer in MRI : a review. NMR Biomed 2001; 14 : 57-64.

Hennig J, Weigel M, Scheffler K. Calculation of Flip Angles for Echo Trains with Predefined Amplitudes with the Extended Phase Graph (EPG)-Algorithm: Principles and Applications to Hyperecho and TRAPS Sequences. 2004; 49 : 527-35.

Heule R, Ganter C, Bieri O. Triple echo steady-state (TESS) relaxometry. Magn Reson Med 2014 ; 71 : 230-7.

Hiba B, Richard N, Hébert LJ, et al. Quantitative assessment of skeletal muscle degeneration in patients with myotonic dystrophy type 1 using MRI. J Magn Reson imaging 2012 ; 35 : 678-85.

Hinojar R, Foote L, Arroyo Ucar E, et al. Native T1 in Discrimination of Acute and Convalescent Stages in Patients With Clinical Diagnosis of Myocarditis. JACC Cardiovasc Imaging 2015; 8 : 37-46.

Hogrel J-Y, Allenbach Y, Canal A, et al. Four-year longitudinal study of clinical and functional endpoints in sporadic inclusion body myositis: implications for therapeutic trials. Neuromuscul Disord $2014 ; 24: 604-10$.

Hogrel J-Y, Barnouin Y, Azzabou N, et al. NMR imaging estimates of muscle volume and intramuscular fat infiltration in the thigh: variations with muscle, gender, and age. Age 2015 ; 37 (3) : 9798 Hollingsworth KG, de Sousa PL, Straub V, Carlier PG. Towards harmonization of protocols for MRI outcome measures in skeletal muscle studies: consensus recommendations from two TREAT-NMD NMR workshops, 2 May 2010, Stockholm, Sweden, 1-2 October 2009, Paris, France. Neuromuscul Disord 2012; 22 Suppl 2 : S54-67.

Hollingsworth KG, Garrood P, Eagle M, et al. Magnetic resonance imaging in Duchenne muscular dystrophy: Longitudinal assessment of natural history over 18 months. Muscle Nerve 2013a; 48 586-8.

Hollingsworth KG, Higgins DM, McCallum M, et al. Investigating the quantitative fidelity of prospectively undersampled chemical shift imaging in muscular dystrophy with compressed sensing and parallel imaging reconstruction. Magn Reson Med 2013b ; 72 : 1016-19

Hooijmans MT, Damon BM, Froeling M, et al. Evaluation of skeletal muscle DTI in patients with duchenne muscular dystrophy. NMR Biomed 2015 ; 28 : 1589-97.

Horn M. 23Na magnetic resonance imaging for the determination of myocardial viability: the status and the challenges. Curr Vasc Pharmacol $2004: 2$ : 329-33.

Hörster I, Weigt-Usinger K, Carmann C, et al. The L-arginine/NO pathway and homoarginine are altered in Duchenne muscular dystrophy and improved by glucocorticoids. Amino Acids $2015 ; 47$ : 1853-63.
Hu HH, Börnert P, Hernando D, et al. ISMRM workshop on fatwater separation: insights, applications and progress in MRI. Magn Reson Med 2012 ; 68: 378-88.

Hu HH, Kan HE. Quantitative proton MR techniques for measuring fat. NMR Biomed 2013; 26 : 1609-29.

Hunter DJ, Zhang W, Conaghan PG, et al. Systematic review of the concurrent and predictive validity of MRI biomarkers in OA. Osteoarthr Cartil 2011; 19 : 557-88.

Jacobi B, Bongartz G, Partovi S, et al. Skeletal muscle BOLD MRI: from underlying physiological concepts to its usefulness in clinical conditions. J Magn Reson imaging 2012 ; 35 : 1253-65.

Janiczek RL, Gambarota G, Sinclair CDJ, et al. Simultaneous T2 and lipid quantitation using IDEAL-CPMG. Magn Reson Med 2011a ; 66 : 1293-302.

Janssen BH, Voet NBM, Nabuurs CI, et al. Distinct disease phases in muscles of facioscapulohumera dystrophy patients identified by MR detected fat Infiltration. PLoS One 2014 ; 9 : e85416.

Jordan BF, Kimpalou JZ, Beghein N, et al. Contribution of oxygenation to BOLD contrast in exercising muscle. Magn Reson Med 2004 ; 52 : 391-6.

Jovicich J, Marizzoni M, Sala-Llonch R, et al. Brain morphometry reproducibility in multi-center 3T MRI studies: a comparison of cross-sectional and longitudinal segmentations. Neuroimage $2013 ; 83: 472-84$

Kamath S, Venkatanarasimha N, Walsh MA, Hughes PM. MRI appearance of muscle denervation. Skeletal Radiol 2008; 37 : 397-404.

Karampinos DC, Baum T, Nardo L, et al. Characterization of the regional distribution of skeletal muscle adipose tissue in type 2 diabetes using chemical shift-based water/fat separation. $J$ Magn Reson imaging 2012; 35 : 899-907.

Karampinos DC, King KF, Sutton BP, Georgiadis JG. Myofiber ellipticity as an explanation for transverse asymmetry of skeletal muscle diffusion MRI in vivo signal. Ann Biomed Eng 2009 ; 37 : 2532-46.

Karampinos DC, King KF, Sutton BP, Georgiadis JG. Intravoxel partially coherent motion technique: characterization of the anisotropy of skeletal muscle microvasculature. J Magn Reson Imaging $2010 ; 31: 942-53$

Karlsson A, Rosander J, Romu T, et al. Automatic and quantitative assessment of regional muscle volume by multi-atlas segmentation using whole-body water-fat MRI. J Magn Reson imaging 2015; 41 : 1558-69.

Kemp GJ, Meyerspeer M, Moser E. Review Article Absolute quantification of phosphorus metabolite concentrations in human muscle in vivo by 31 P MRS : a quantitative review. NMR Biomed 2007; 20 : 555-65

Kemp GJ, Taylor DJ, Dunn JF, et al. Cellular energetics of dystrophic muscle. J Neurol Sci 1993; 116: 201-6.

Kharraz Y, Guerra J, Pessina P, et al. Understanding the process of fibrosis in Duchenne muscular dystrophy. Bio Med Res Int 2014 ; doi: 10.1155/2014/965631.

Kim HK, Laor T, Horn PS, et al. T2 mapping in Duchenne muscular dystrophy: distribution of disease activity and correlation with clinical assessments. Radiology $2010 ; 255$ : 899-908.

Kim HK, Serai S, Lindquist D, et al. Quantitative Skeletal Muscle MRI: Part 2, MR Spectroscopy and T2 Relaxation Time Mapping-Comparison Between Boys With Duchenne Muscular Dystrophy and Healthy Boys. Amer J of Roentgenol 2015; 205 : 216-23.

Kim HK, Serai S, Merrow AC, et al. Objective measurement of minimal fat in normal skeletal muscles of healthy children using T2 relaxation time mapping (T2 maps) and MR spectroscopy. Pediatr Radiol 2014 ; 44 : 149-57.

Klingler W, Jurkat-Rott K, Lehmann-Horn F, Schleip R. The role of fibrosis in Duchenne muscular dystrophy. Acta Myol 2012 ; $31: 184-95$.

Kornegay JN, Bogan JR, Bogan DJ, et al. Canine models of Duchenne muscular dystrophy and their use in therapeutic strategies. Mamm genome 2012 ; 23 : 85-108

Kusmia S, Eliav U, Navon G, Guillot G. DQF-MT MRI of connective tissues: application to tendon and muscle. Magma $2013 ; 26$ : 203-14. 
Lamminen AE. Magnetic resonance imaging of primary skeletal muscle diseases: patterns of distribution and severity of involvement. Br J Radiol 1990 ; 63 : 946-50.

Lavini $\mathrm{C}$, de Jonge MC, van de Sande MGH, et al. Pixel-by-pixel analysis of DCE MRI curve patterns and an illustration of its application to the imaging of the musculoskeletal system. Magn Reson Imaging $2007 ; 25: 604-12$.

Le Guiner C, Montus M, Servais L, et al. Forelimb treatment in a large cohort of dystrophic dogs supports delivery of a recombinant AAV for exon skipping in Duchenne patients. Mol Ther 2014 ; $22: 1923-35$

Lebel RM, Wilman AH. Transverse relaxometry with stimulated echo compensation. Magn Reson Med 2010 ; 64 : 1005-14.

Lebon V, Brillault-Salvat C, Bloch G, et al. Evidence of muscle BOLD effect revealed by simultaneous interleaved gradient-echo NMRI and myoglobin NMRS during leg ischemia. Magn Reson Med $1998 ; 40: 551-8$

Ledermann H-P, Schulte A-C, Heidecker H-G, et al. Blood oxygenation level-dependent magnetic resonance imaging of the skeletal muscle in patients with peripheral arterial occlusive disease. Circulation 2006 ; 113 : 2929-35.

Lee YH, Lee HS, Lee HE, et al. Whole-Body Muscle MRI in Patients with Hyperkalemic Periodic Paralysis Carrying the SCN4A Mutation T704M: Evidence for Chronic Progressive Myopathy with Selective Muscle Involvement. J Clin Neurol 2015; 11 : 331-8.

Leong KM, Lau P, Ramadan S. Utilisation of MR spectroscopy and diffusion weighted imaging in predicting and monitoring of breast cancer response to chemotherapy. $J$ Med Imaging Radiat Oncol 2015; 59 : 268-77.

Leroy-Willig a., Willig TN, Henry-Feugeas MC, et al. Body composition determined with MR in patients with Duchenne muscular dystrophy, spinal muscular atrophy, and normal subjects. Magn Reson Imaging 1997; 15 : 737-44.

Lerski RA, de Certaines JD, Duda D, et al. Application of texture analysis to muscle MRI: 2 - technical recommendations. EPJ Nonlinear Biomed Phys 2015 ; 3 : 1-20.

Li C, Magland JF, Rad HS, et al. Comparison of optimized softtissue suppression schemes for ultrashort echo time MRI. Magn Reson Med 2012 ; 68: 680-9

Li K, Dortch RD, Kroop SF, et al. A rapid approach for quantitative magnetization transfer imaging in thigh muscles using the pulsed saturation method. Magn Reson Imaging 2015; 33 : 709-17.

Li K, Dortch RD, Welch EB, et al. Multi-parametric MRI characterization of healthy human thigh muscles at $3.0 \mathrm{~T}$ - relaxation, magnetization transfer, fat/water, and diffusion tensor imaging. NMR Biomed $2014 ; 27$ : 1070-84.

Liu G-C, Jong Y-J, Chiang C-H, Jaw T-S. Duchenne muscular Dystrophy: MR grading system with functional correlation. Radiology $1993 ; 186: 475-80$.

Lodi R, Kemp GJ, Muntoni F, et al. Reduced cytosolic acidification during exercise suggests defective glycolytic activity in skeletal muscle of patients with Becker muscular dystrophy. An in vivo 31P magnetic resonance spectroscopy study. Brain 1999; 122 : 121-30

Longo R, Pollesello P, Ricci C, et al. Proton MR spectroscopy in quantitative in vivo determination of fat content in human liver steatosis. J Magn Reson Imaging 1995 ; 5 : 281-85.

Loughran T, Higgins DM, McCallum M, et al. Improving highly accelerated fat fraction measurements for clinical trials in muscular dystrophy: origin and quantitative effect of $\mathrm{R} 2 *$ changes. Radiology $2015 ; 275: 570-78$.

Lustig M, Donoho DL, Santos JM, Pauly JM. Compressed Sensing MRI. IEEE Signal Processing Magazine 2008 ; 25 : 72-85.

Lynn S, Aartsma-Rus A, Bushby K, et al. Measuring clinical effectiveness of medicinal products for the treatment of Duchenne muscular dystrophy. Neuromuscul Disord 2015 ; 25 : 96-105.

Ma J. Dixon techniques for water and fat imaging. $J$ Magn Reson imaging 2008; 28 : 543-58.

Madelin G, Regatte RR. Biomedical applications of sodium MRI in vivo. J Magn Reson imaging 2013 ; 38 : 511-29.
Maillard SM, Jones R, Owens C, et al. Quantitative assessment of MRI T2 relaxation time of thigh muscles in juvenile dermatomyositis. Rheumatology (Oxford) $2004 ; 43$ : 603-8.

Martins Bach A, Matot B, Wary C, et al. Non-invasive NMR study of the mouse model for centronuclear myopathy with mutation in the dynamin-2 gene. Neuromuscul Disord 2015; $25: 275$. Martins-Bach AB, Malheiros J, Matot B, et al. Quantitative T2 combined with texture analysis of nuclear magnetic resonance images identify different degrees of muscle involvement in three mouse models of muscle dystrophy: mdx, Largemyd and mdx/ Largemyd. PLoS One 2015; 10 : e0117835.

Marty B, Vignaud A, Greiser A, et al. BLOCH equations-based reconstruction of myocardium $\mathrm{t}$ maps from modified look-locker inversion recovery sequence. PLoS One 2015; 10 : e0126766. Marty B, Baudin PY, Reyngoudt H, et al. Simultaneous muscle water T2 and fat fraction mapping using transverse relaxometry with stimulated echo compensation. NMR Biomed 2016; 29 : 431-43.

Mathur S, Vohra RS, Germain SA, et al. Changes in muscle T2 and tissue damage after downhill running in mdx mice. Muscle Nerve $2011 ; 43$ : 878-86.

Mattei JP, Le Fur Y, Cuge N, et al. Segmentation of fascias, fat and muscle from magnetic resonance images in humans: The DISPIMAG software. Magn Reson Mater Physics, Biol Med 2006 ; 19: 275-79

Mayhew A, Mazzone ES, Eagle M, et al. Development of the Performance of the Upper Limb module for Duchenne muscular dystrophy. Dev Med Child Neurol 2013 ; 55 : 1038-45.

Mazzone E, De Sanctis R, Fanelli L, et al. Hammersmith Functional Motor Scale and Motor Function Measure-20 in non ambulant SMA patients. Neuromuscul Disord $2014 ; 24$ : 347-52.

Mazzone ES, Vasco G, Palermo C, et al. A critical review of functional assessment tools for upper limbs in Duchenne muscular dystrophy. Dev Med Child Neurol 2012 ; 54 : 879-85.

McCullough MB, Domire ZJ, Reed AM, et al. Evaluation of muscles affected by myositis using magnetic resonance elastography. Muscle Nerve 2011 ; 43 : 585-90.

McDonald CM, Henricson EK, Abresch RT, et al. The 6-minute walk test and other clinical endpoints in duchenne muscular dystrophy: reliability, concurrent validity, and minimal clinically important differences from a multicenter study. Muscle Nerve 2013; 48 : 357-68.

McIntosh LM, Baker RE, Anderson JE. Magnetic resonance imaging of regenerating and dystrophic mouse muscle. Biochem Cell Biol 1998; 76 : 532-41.

Mead AF, Petrov M, Malik AS, et al. Diaphragm remodeling and compensatory respiratory mechanics in a canine model of Duchenne muscular dystrophy. J Appl Physiol 2014; 116 : 807-15.

Mercuri E, Muntoni F. Muscular dystrophy: new challenges and review of the current clinical trials. Curr Opin Pediatr 2013; 25 : 701-7.

Meyer RA, Towse TF, Reid RW, et al. BOLD MRI mapping of transient hyperemia in skeletal muscle after single contractions. NMR Biomed $2004 ; 17: 392-8$

Miller CA, Naish JH, Bishop P, et al. Comprehensive Validation of Cardiovascular Magnetic Extracellular Volume. Circ Cardiovasc Imaging $2013 ; 6: 373-83$.

Mills KL, Tamnes CK Methods and considerations for longitudinal structural brain imaging analysis across development. Dev Cogn Neurosci $2014 ; 9$ : 172-90.

Mitsiopoulos N, Baumgartner RN, Heymsfield SB, et al. Cadaver validation of skeletal muscle measurement by magnetic resonance imaging and computerized tomography. J Appl Physiol 1998; $85: 115-22$.

Moon JC, Messroghli DR, Kellman P, et al. Myocardial T1 mapping and extracellular volume quantification: a Society for Cardiovascular Magnetic Resonance (SCMR) and CMR Working Group of the European Society of Cardiology consensus statement. J Cardiovasc Magn Reson 2013; 15 : 92-103.

Morrison C, Henkelman RM. A model for magnetization transfer in tissues. Magn Reson Med 1995 ; 33 : 475-82. 
Morrow JM, Sinclair CDJ, Fischmann A, et al. MRI biomarker assessment of neuromuscular disease progression: a prospective observational cohort study. Lancet Neurol $2015 ; 4422$ : 1-13.

Morrow JM, Sinclair CDJ, Fischmann A, et al. Reproducibility, and age, body-weight and gender dependency of candidate skeletal muscle MRI outcome measures in healthy volunteers. Eur Radiol $2014 ; 24: 1610-20$

Morse CI, Degens H, Jones DA. The validity of estimating quadriceps volume from single MRI cross-sections in young men. Eur $J$ Appl Physiol 2007 ; 100 : 267-74.

Muntoni F, Wood MJA. Targeting RNA to treat neuromuscular disease. Nat Rev Drug Discov 2011 ; 10 : 621-37.

Nardo L, Karampinos DC, Lansdown DA, et al. Quantitative assessment of fat infiltration in the rotator cuff muscles using water-fat MRI. J Magn Reson imaging 2014 ; 39 : 1178-85.

Nelson MD, Rader F, Tang X, et al. PDE5 inhibition alleviates functional muscle ischemia in boys with Duchenne muscular dystrophy. Neurology 2014; 82 : 2085-91

Newman RJ, Bore PJ, Chan L, et al. (1982) Nuclear magnetic resonance studies of forearm muscle in Duchenne dystrophy. $\mathrm{Br}$ Med J (Clin Res Ed) 284 : 1072-74.

Nozaki T, Tasaki A, Horiuchi S, et al. Quantification of Fatty Degeneration Within the Supraspinatus Muscle by Using a 2-Point Dixon Method on 3-T MRI. Amer J Roentgenol 2015; 205 : 116-22.

Ogawa S, Lee TM, Kay AR, Tank DW. Brain magnetic resonance imaging with contrast dependent on blood oxygenation. Proc Nat Acad Sci USA 1990 ; 87 : 9868-72

Pacak CA, Walter GA, Gaidosh G, et al. Long-term skeletal muscle protection after gene transfer in a mouse model of LGMD-2D. Mol Ther 2007 ; 15: 1775-81.

Pane M, Mazzone ES, Sormani MP, et al. 6 Minute walk test in Duchenne MD patients with different mutations: 12 month changes. PLoS One 2014 ; 9 : e83400.

Park J, Wicki J, Knoblaugh SE, et al. Multi-parametric MRI at 14T for muscular dystrophy mice treated with AAV vector-mediated gene therapy. PLoS One 2015; 10 : e0124914.

Park JH, Vansant JP, Kumar NG, et al. Dermatomyositis: correlative MR imaging and P-31 MR spectroscopy for quantitative characterization of inflammatory disease. Radiology 1990; 177 : 473-9.

Partovi S, Schulte A-C, Aschwanden M, et al. Impaired skeletal muscle microcirculation in systemic sclerosis. Arthritis Res Ther 2012; 14 : R209.

Partovi S, von Tengg-Kobligk H, Bhojwani N, et al. Advanced Noncontrast MR Imaging in Musculoskeletal Radiology. Radiol Clin North Am 2015; 53 : 549-67.

Parzy E, Fromes Y, Thiaudiere E, Carlier PG. Rapid Communication Refinement of cardiac NMR imaging in awake hamsters: proof of feasibility and characterization of cardiomyopathy. NMR Biomed 2007; $20: 615-23$.

Périé S, Trollet C, Mouly V, et al. Autologous myoblast transplantation for oculopharyngeal muscular dystrophy: a phase I/IIa clinical study. Mol Ther 2014; $22: 219-25$

Pichiecchio A, Uggetti C, Egitto MG, et al. Quantitative MR evaluation of body composition in patients with Duchenne muscular dystrophy. Eur Radiol $2002 ; 12: 2704-9$.

Polak JF, Jolesz FA, Adams DF (1988) Magnetic resonance imaging of skeletal muscle. Prolongation of T1 and T2 subsequent to denervation. Invest Radiol 23 : 365-9.

Ponrartana S, Ramos-Platt L, Wren TAL, et al. Effectiveness of diffusion tensor imaging in assessing disease severity in Duchenne muscular dystrophy: preliminary study. Pediatr Radiol $2015 ; 45$ : 582-9.

Pratt SJ, Xu S, Mullins RJ, Lovering RM. Temporal changes in magnetic resonance imaging in the $\mathrm{mdx}$ mouse. BMC Res Notes $2013 ; 6: 262$

Pruessmann KP, Weiger M, Scheidegger MB, Boesiger P. SENSE sensitivity encoding for fast MRI. Magn Reson Med 1999; 42 : 952-62.

Qi J, Olsen NJ, Price RR, et al. Diffusion-weighted imaging of inflammatory myopathies: polymyositis and dermatomyositis. $J$ Magn Reson Imaging 2008 ; 27 : 212-7.
Ravaglia S, Pichiecchio A, Ponzio M, et al. Changes in skeletal muscle qualities during enzyme replacement therapy in late-onset type II glycogenosis: temporal and spatial pattern of mass vs. strength response. J Inherit Metab Dis 2010 ; 33 : 737-45.

Raynaud JS, Duteil S, Vaughan JT, et al. Determination of skeletal muscle perfusion using arterial spin labeling NMRI: Validation by comparison with venous occlusion plethysmography. Magn Reson Med 2001 ; 46 : 305-11.

Redmond OM, Stack JP, O'Connor NG, et al. 31P MRS as an early prognostic indicator of patient response to chemotherapy. Magn Reson Med 1992 ; 25 : 30-44.

Ringleb SI, Bensamoun SF, Chen Q, et al. Applications of magnetic resonance elastography to healthy and pathologic skeletal muscle. J Magn Reson imaging 2007 ; 25 : 301-9.

Robson MD, Bydder GM. Clinical ultrashort echo time imaging of bone and other connective tissues. NMR Biomed 2006; 19 : 765-80.

Rooney WD, Pollaro JR, Forbes SC, et al. Application of the Extended Phas e Graph Technique to Improve T2 Quantitation Across Sites. In: Proceedings ISMRM 54192011.

Saab G, Thompson RT, Marsh GD. Effects of exercise on muscle transverse relaxation determined by MR imaging and in vivo relaxometry. J Appl Physiol $2000 ; 88$ : 226-33.

Saab G, Thompson RT, Marsh GD. Multicomponent T 2 Relaxation of In Vivo Skeletal Muscle. Magn Reson Med 1999; 157 : 150-7

Schewzow K, Fiedler GB, Meyerspeer M, et al. Dynamic ASL and T2*-weighted MRI in exercising calf muscle at $7 \mathrm{~T}$ : A feasibility study. Magn Reson Med 2014 ; 73 : 1190-5

Schmitt P, Griswold MA, Jakob PM, et al. Inversion recovery TrueFISP: quantification of T(1), T(2), and spin density. Magn Reson Med 2004 ; 51 : 661-7.

Schwenzer NF, Martirosian P, Machann J, et al. (2009a) Aging effects on human calf muscle properties assessed by MRI at 3 Tesla. J Magn Reson imaging 29 : 1346-54.

Schwenzer NF, Steidle G, Martirosian P, et al. (2009b) Diffusion tensor imaging of the human calf muscle: distinct changes in fractional anisotropy and mean diffusion due to passive muscle shortening and stretching. NMR Biomed 22 : 1047-53.

Scott E, Eagle M, Mayhew A, et al. Development of a functional assessment scale for ambulatory boys with Duchenne muscular dystrophy. Physiother Res Int 2012; 17 : 101-9.

Scotter EL, Shaw CE. Neuromuscular disease: new insights and avenues for therapy. Lancet Neurol 2013; 12 : 13-5.

Seferian AM, Moraux A, Annoussamy M, et al. Upper limb strength and function changes during a one-year follow-up in nonambulant patients with Duchenne Muscular Dystrophy: an observational multicenter trial. PLoS One 2015a ; 10 : e0113999.

Seferian AM, Moraux A, Canal A, et al. Upper limb evaluation and one-year follow up of non-ambulant patients with spinal muscular atrophy: an observational multicenter trial. PLoS One 2015b ; 10 : e0121799.

Servais L, Deconinck N, Moraux A, et al. Innovative methods to assess upper limb strength and function in non-ambulant Duchenne patients. Neuromuscul Disord 2013; 23 : 139-48.

Shin HJ, Baek H-M, Ahn J-H, et al. Prediction of pathologic response to neoadjuvant chemotherapy in patients with breast cancer using diffusion-weighted imaging and MRS. NMR Biomed 2012; $25: 1349-59$.

Sigmund EE, Novikov DS, Sui D, et al. Time-dependent diffusion in skeletal muscle with the random permeable barrier model (RPBM) : application to normal controls and chronic exertional compartment syndrome patients. NMR Biomed 2014; 27 : 519-28.

Sigmund EE, Sui D, Ukpebor O, et al. Stimulated echo diffusion tensor imaging and SPAIR T2 - weighted imaging in chronic exertional compartment syndrome of the lower leg muscles. $J$ Magn Reson Imaging 2013 ; 38 : 1073-82.

Sinclair CDJ, Morrow JM, Miranda MA, et al. Skeletal muscle MRI magnetisation transfer ratio reflects clinical severity in peripheral neuropathies. J Neurol Neurosurg Psychiatry 2012 ; 83 : 29-32. Sinclair CDJ, Samson RS, Thomas DL, et al. Quantitative magnetization transfer in in vivo healthy human skeletal muscle at $3 \mathrm{~T}$. Magn Reson Med 2010 ; 64 : 1739-48. 
Siu AG, Ramadeen A, Hu X, et al. Characterization of the ultrashort-TE (UTE) MR collagen signal. NMR Biomed 2015; 28 : 1236-44.

Smeulders MJC, van den Berg S, Oudeman J, et al. Reliability of in vivo determination of forearm muscle volume using $3.0 \mathrm{~T}$ magnetic resonance imaging. J Magn Reson imaging 2010 ; 31 : 1252-5.

Sproule DM, Montgomery MJ, Punyanitya M, et al. Thigh muscle volume measured by magnetic resonance imaging is stable over a 6-month interval in spinal muscular atrophy. $J$ Child Neurol $2011 ; 26: 1252-9$

Statland, Jeffrey, Donlin-Smith, Colleen M, Tapscott, Stephen J, Van der Maarel, Silvère M, Tawil, Rabi Multiplex screen of serum biomarkers in facioscapulohumeral muscular mystrophy. J Neuromuscul Dis $2014 ; 1: 181-90$

Stejskal EO, Tanner JE (1965) Spin diffusion measurements: spin echoes in the presence of a time-dependant field gradient. $J$ Chem Phys $42: 288-292$

Strandberg S, Wretling M-L, Wredmark T, Shalabi A. Reliability of computed tomography measurements in assessment of thigh muscle cross-sectional area and attenuation. BMC Med Imaging $2010 ; 10: 18$.

Straub V, Donahue KM, Allamand V, et al. Contrast agentenhanced magnetic resonance imaging of skeletal muscle damage in animal models of muscular dystrophy. Magn Reson Med 2000 ; $44: 655-9$

Strimbu K, Tavel JA. What are biomarkers? Curr Opin HIV AIDS 2010 ; 5 : 463-6.

Tanaka NI, Kanehisa H. Applicability of Single Muscle CSA for Predicting Segmental Muscle Volume in Young Men. Int J Sports Med 2014 ; 35 : 608-14.

Tardif-de Géry S, Vilquin J, Carlier P, et al. Muscular transverse relaxation time measurement by magnetic resonance imaging at 4 Tesla in normal and dystrophic dy/dy and dy(2j)/dy(2j) mice. Neuromuscul Disord 2000 ; 10 : 507-13.

Tasca G, Pescatori M, Monforte M, et al. Different molecular signatures in magnetic resonance imaging-staged facioscapulohumeral muscular dystrophy muscles. PLoS One 2012; 7 : e38779. Thibaud J, Matot B, Barthélémy I, et al. Diaphragm structural abnormalities revealed by NMR imaging in the dystrophic dog. Neuromuscul Disord 2013; 23 : 809-10.

Thibaud J-L, Azzabou N, Barthelemy I, et al. Comprehensive longitudinal characterization of canine muscular dystrophy by serial NMR imaging of GRMD dogs. Neuromuscul Disord 2012; 22 Suppl $2:$ 85-99.

Thibaud J-L, Monnet A, Bertoldi D, et al. Characterization of dystrophic muscle in golden retriever muscular dystrophy dogs by nuclear magnetic resonance imaging. Neuromuscul Disord $2007 ; 17: 575-84$.

Thomas GD. Functional muscle ischemia in Duchenne and Becker muscular dystrophy. Front Physiol 2013 ; 4 : 381-6.

Thomas MS, Newman D, Leinhard OD, et al. Test-retest reliability of automated whole body and compartmental muscle volume measurements on a wide bore 3T MR system. Eur Radiol 2014 ; 24 2279-91.

Thulborn K, Soffe N, Kadda G. (1981) Simultaneous in vivo measurement of oxygen utilization and high-energy phosphate metabolism in rabbit skeletal muscle by multinuclear $1 \mathrm{H}$ and 31P NMR. J Magn Reson 1981; 45 : 362-66.

Tofts PS. Modeling tracer kinetics in dynamic Gd-DTPA MR imaging. J Magn Reson Imaging 1997; 7 : 91-101.

Tofts PS. Quantitative MRI of the brain: measuring changes caused by disease. John Wiley 2003.

Tofts PS, Brix G, Buckley DL, et al. Contrast-wnhanced T 1 weighted MRI of a diffusable tracer: standardized quantities and symbols. J Magn Reson Imaging 1999 ; 232 : 223-32.

Torriani M, Townsend E, Thomas BJ, et al. Lower leg muscle involvement in Duchenne muscular dystrophy: an MR imaging and spectroscopy study. Skeletal Radiol 2012; 41 : 437-45.

Tosetti M, Linsalata S, Battini R, et al. Muscle metabolic alterations assessed by 31-phosphorus magnetic resonance spectroscopy in mild Becker muscular dystrophy. Muscle Nerve 2011 ; 44 : 816-9.
Touznik A, Lee JJA, Yokota T. New developments in exon skipping and splice modulation therapies for neuromuscular diseases. Expert Opin Biol Ther 2014 ; 14 : 809-19.

Tyler DJ, Robson MD, Henkelman RM, et al. Magnetic resonance imaging with ultrashort TE (UTE) PULSE sequences: technical considerations. J Magn Reson imaging 2007 ; 25 : 279-89. Vasan RS. Biomarkers of cardiovascular disease: molecular basis and practical considerations. Circulation $2006 ; 113$ : 2335-62. Vignaud A, Guillot G, Caldas de Almeida Araújo E, Carlier PG. NMR imaging of short T2-components in skeletal muscle tissue. Neuromuscul Disord $2014 ; 24: 837$.

Vohra R, Accorsi A, Kumar A, et al. Magnetic Resonance Imaging Is Sensitive to Pathological Amelioration in a Model for LamininDeficient Congenital Muscular Dystrophy (MDC1A). PLoS One 2015a ; 10 : e0138254

Vohra RS, Lott D, Mathur S, et al. Magnetic Resonance Assessment of Hypertrophic and Pseudo-Hypertrophic Changes in Lower Leg Muscles of Boys with Duchenne Muscular Dystrophy and Their Relationship to Functional Measurements. PLoS One 2015b ; 10 : e0128915.

Vohra RS, Mathur S, Bryant ND, et al. Age-related T2 changes in hindlimb muscles of mdx mice. Muscle Nerve 2015c; 53 (1): 84-90.

Voit $\mathrm{T}$. The challenge of making therapies happen for neuromuscular diseases. Neuromuscul Disord 2014 ; 24 : 918-9.

Voit T, Topaloglu H, Straub V, et al. Safety and efficacy of drisapersen for the treatment of Duchenne muscular dystrophy (DEMAND II) : an exploratory, randomised, placebo-controlled phase 2 study. Lancet Neurol 2014 ; 13 : 987-96.

Wagner KR, Fleckenstein JL, Amato AA, et al. A phase I/IItrial of MYO-029 in adult subjects with muscular dystrophy. Ann Neurol 2008; 63: 561-71.

Walker UA. Imaging tools for the clinical assessment of idiopathic inflammatory myositis. Curr Opin Rheumatol 2008; 20 656-61.

Walter G, Cordier L, Bloy D, Sweeney HL. Noninvasive monitoring of gene correction in dystrophic muscle. Magn Reson Med 2005 ; 54 : 1369-76.

Wang J, Fan Z, Vandenborne K, et al. A computerized MRI biomarker quantification scheme for a canine model of Duchenne muscular dystrophy. Int J Comput Assist Radiol Surg 2013; 8 (5) : 763-74

Wang K, Yu H, Brittain J. K space water fat decomposition with $\mathrm{T}^{*}$ estimation and multifrequency fat spectrum modeling for ultrashort echo time imaging. J Magn Reson Imaging 2010 ; 31 (4) : 1027-34.

Wary C, Azzabou N, Giraudeau C, et al. Quantitative NMRI and NMRS identify augmented disease progression after loss of ambulation in forearms of boys with Duchenne muscular dystrophy. NMR Biomed 2015a ; 28 : 1150-62.

Wary C, Azzabou N, Giraudeau C, et al. Quantitative NMRI and NMRS identify augmented disease progression after loss of ambulation in forearms of boys with Duchenne muscular dystrophy. NMR Biomed 2015b ; 28 : 1150-62.

Wary C, Nadaj-Pakleza A, Laforêt P, et al. Investigating glycogenosis type III patients with multi-parametric functional NMR imaging and spectroscopy. Neuromuscul Disord 2010 ; 20 : 548-58 Wary C, Naulet T, Thibaud J-L, et al. Splitting of Pi and other 31P NMR anomalies of skeletal muscle metabolites in canine muscular dystrophy. NMR Biomed 2012; 25 : 1160-9.

Weigel M. Extended phase graphs: Dephasing, RF pulses, and echoes - pure and simple. J Magn Reson Imaging 2014.

Willcocks RJ, Arpan I a, Forbes SC, et al. Longitudinal measurements of MRI-T2 in boys with Duchenne muscular dystrophy: effects of age and disease progression. Neuromuscul Disord $2014 ; 24: 393-401$.

Williams SA, Reid S, Elliott $C$, et al. Muscle volume alterations in spastic muscles immediately following botulinum toxin type-A treatment in children with cerebral palsy. Dev Med Child Neurol $2013 ; 55: 813-20$.

Willis TA, Hollingsworth KG, Coombs A, et al. Quantitative muscle MRI as an assessment tool for monitoring disease progression in LGMD2I: a multicentre longitudinal study. PLoS One 2013 ; 8 : e70993. 
Wishnia A, Alameddine H, Tardif de Gery S, Leroy-Willio A. Use of magnetic resonance imaging for noninvasive characterization and follow-up of an experimental injury to normal mouse muscles. Neuromuscul Disord 2001; 11: 50-55

Wokke BH, Bos C, Reijnierse M, et al. Comparison of dixon and T1-weighted MR methods to assess the degree of fat infiltration in duchenne muscular dystrophy patients. J Magn Reson imaging $2013 ; 38$ : 619-24.

Wokke BH, Hooijmans MT, van den Bergen JC, et al. Muscle MRS detects elevated PDE/ATP ratios prior to fatty infiltration in Becker muscular dystrophy. NMR Biomed 2014a ; 27 : 1371-7. Wokke BH, van den Bergen JC, Versluis MJ, et al. Quantitative MRI and strength measurements in the assessment of muscle quality in Duchenne muscular dystrophy. Neuromuscul Disord 2014b ; 24 : 409-16

Wokke $\mathrm{BH}$, van den Bergen JC, Hooijmans MT, et al. T2 relaxation times are increased in skeletal muscle of DMD but not BMD patients. Muscle Nerve 2016; 53 : 38-43.

Wren TA, Bluml S, Tseng-Ong L, Gilsanz V. Three-point technique of fat quantification of muscle tissue as a marker of disease progression in Duchenne muscular dystrophy: preliminary study. Amer J Roentgenol 2008; 190 : W8-12.

Wynn TA. Cellular and molecular mechanisms of fibrosis. J Pathol $2008 ; 214: 199-210$

Xi W, Perdanasari AT, Ong Y, et al. Objective breast volume, shape and surface area assessment: a systematic review of breast measurement methods. Aesthetic Plast Surg 2014; 38 1116-30.

Yanagisawa O, Shimao D, Maruyama K, et al. Diffusion-weighted magnetic resonance imaging of human skeletal muscles: genderage- and muscle-related differences in apparent diffusion coefficient. Magn Reson Imaging 2009 ; 27 : 69-78.
Yao L, Gai N. Fat-corrected T2 measurement as a marker of active muscle disease in inflammatory myopathy. Am $J$ Roentgenol 2012; 198 : 475-81.

Yarnykh VL. Actual flip-angle imaging in the pulsed steady state: a method for rapid three-dimensional mapping of the transmitted radiofrequency field. Magn Reson Med 2007 ; 57 : 192-200.

Yarnykh VL, Tartaglione EV, Ioannou GN. Fast macromolecular proton fraction mapping of the human liver in vivo for quantitative assessment of hepatic fibrosis. NMR Biomed 2015; 28 : 1716-25.

Yasuda T, Loenneke JP, Thiebaud RS, Abe T. Effects of detraining after blood flow-restricted low-intensity concentric or eccentric training on muscle size and strength. J Physiol Sci 2015; 65 : 139-44.

Yokota T, Lu Q-L, Partridge T, et al. Efficacy of systemic morpholino exon-skipping in Duchenne dystrophy dogs. Ann Neurol 2009 ; 65 : 667-76

Younkin DP, Berman P, Sladky J, et al. 31P NMR studies in Duchenne muscular dystrophy: age-related metabolic changes. Neurology 1987 ; 37 : 165-69

Zaraiskaya T, Kumbhare D, Noseworthy MD. Diffusion tensor imaging in evaluation of human skeletal muscle injury. J Magn Reson Imaging $2006 ; 24$ : 402-8.

Zheng J, An H, Coggan AR, et al. Noncontrast skeletal muscle oximetry. Magn Reson Med 2013 ; 71 : 318-25.

Zheng J, Hasting MK, Zhang X, et al. A pilot study of regional perfusion and oxygenation in calf muscles of individuals with diabetes with a non-invasive measure. J Vasc Surg 2014; 59: 419-26.

Zhou L, Lu H. Targeting fibrosis in Duchenne muscular dystrophy. $J$ Neuropathol Exp Neurol 2010 ; 69 : 771-6. 